\title{
Nut consumption for vascular health and cognitive function
}

\author{
Jayne A. Barbour ${ }^{1}$, Peter R. C. Howe ${ }^{2}$, Jonathan D. Buckley ${ }^{1}$, Janet Bryan ${ }^{1}$ and Alison M. Coates ${ }^{1 *}$ \\ ${ }^{1}$ Nutrition Physiology Research Centre, Sansom Institute for Health Research, University of South Australia, GPO Box 2471, \\ Adelaide, SA 5001, Australia \\ ${ }^{2}$ Clinical Nutrition Research Centre, University of Newcastle, Callaghan, NSW 2308, Australia
}

\section{Abstract}

Nuts are rich in many nutrients that can benefit multiple cardiometabolic functions, including arterial compliance, blood pressure, inflammation, glucoregulation and endothelial vasodilatation. Impaired vasodilatation may contribute to impaired cognitive performance due to poor cerebral perfusion. The present narrative review examines associations between nut consumption, vascular health and cognitive function. It includes a systematic search which identified seventy-one epidemiological or intervention studies in which effects of chronic nut consumption on blood pressure, glucoregulation, endothelial vasodilator function, arterial compliance, inflammatory biomarkers and cognitive performance were evaluated. Weighted mean changes were estimated where data were available; they indicate that nut consumption reduces blood pressure and improves glucoregulation, endothelial vasodilator function and inflammation, whilst a limited number of studies suggest that nut consumption may also improve cognitive performance. Further clinical trials are warranted to explore relationships between nut consumption, endothelial function and cognitive function.

\section{Key words: Nuts: Endothelial function: Inflammation: Vascular function: Cognition}

\section{Introduction}

CVD and cognitive impairment are growing worldwide health concerns, particularly as populations age ${ }^{(1,2)}$. In 2006, the worldwide prevalence of Alzheimer's disease was estimated at 26.6 million and by 2050 this is predicted to quadruple ${ }^{(3)}$. Increasing evidence suggests that $\mathrm{CVD}$, the metabolic syndrome, hypertension, obesity and type 2 diabetes are associated with diminished cognitive functioning and an increase in all types of dementia ${ }^{(4)}$. These cognitive changes may be mediated through compromises in the structural and functional integrity of cerebral blood vessels. Cognitive performance refers collectively to mental processes including attention, memory, language, problem solving and decision making. Understanding the mechanisms for regulating cognitive functions is important to reduce the impact of declining cognition in older adults. Interventions that slow or prevent this condition are valuable and have become a health priority ${ }^{(5)}$. One of the mechanisms by which cognitive performance can be improved and cognitive decline delayed may be through maintenance of blood vessel health and improvement in blood flow to the brain ${ }^{(6,7)}$. Impaired vasodilatation contributes to reduced cognitive performance, due to poor peripheral and cerebral perfusion ${ }^{(8)}$. Endothelial cells line blood vessels (including those in the brain); thus maintaining cerebral vascular function to ensure normal regulation of cerebral blood flow for the delivery of nutrients is essential to maintain endothelial cell integrity ${ }^{(9)}$.

It has been hypothesised that inflammation may contribute to cognitive decline ${ }^{(10)}$ and to CVD processes ${ }^{(11)}$. This may be a result of endothelial dysfunction ${ }^{(12,13)}$ associated with reduced NO bioavailability. NO is an important vasodilator, produced from L-arginine by endothelial NO synthase $^{(14)}$. Early phases of atherosclerosis involve the adhesion of circulating monocytes to the endothelium (inner lining of blood vessel walls) and their migration to the intima layer. This is a complex disease process mediated by inflammatory responses that involve cytokine production and up-regulation of adhesion molecules such as intercellular adhesion molecule-1 (ICAM-1), vascular cell adhesion molecule-1 (VCAM-1) and E-selectin. An increase in inflammatory cytokines (for example, C-reactive protein (CRP) and IL-6) have been found to be independent predictors of CVD and type 2 diabetes ${ }^{(15)}$. The endothelium is crucial for the maintenance of vascular tone and vascular structure; endothelial dysfunction predisposes individuals to complications of atherosclerosis by increasing blood

\footnotetext{
Abbreviations: ALA, $\alpha$-linolenic acid; CRP, C-reactive protein; ICAM-1, intercellular adhesion molecule-1; PREDIMED, PREvencion con DIeta MEDiterranea
} VCAM-1, vascular cell adhesion molecule-1. 
pressure and arterial stiffness, characterised by increased pulse-wave velocity and an increase in augmentation index. Endothelial function declines with age but is also adversely affected by hypertension, hyperglycaemia, dyslipidaemia and obesity, individually or collectively known as the metabolic syndrome ${ }^{(16)}$.

The principal energy source for the brain is glucose, which must be supplied continuously due to a limited storage capacity ${ }^{(17)}$. In addition, a range of nutrients and substrates including oxygen needs to be delivered via the blood $^{(18)}$; hence cerebral blood flow and substrate transport across the blood-brain barrier are primary determinants of brain function ${ }^{(19)}$. There is a growing interest in the role of nutrition in the causation and prevention of age-related cognitive decline and dementia; more research is needed to understand mechanisms for cognitive decline and possible delay.

As shown in Table 1, nuts contain a range of nutrients with potential health benefits including improved glucose control and insulin sensitivity ${ }^{(20,21)}$. Despite the high fat content of nuts, nut consumption has not been shown to increase body weight; instead it is associated with improved weight control ${ }^{(15,22)}$. There is a substantial body of evidence demonstrating lipid-lowering effects of nut consumption ${ }^{(23)}$ and large epidemiological studies have consistently revealed an association between frequent nut consumption and reduced incidence of $\mathrm{CHD}^{(24)}$. A meta-analysis of thirteen intervention studies using walnuts $^{(25)}$ and a pooled analysis of twenty-five intervention studies with a range of nuts indicated a consistent cholesterol-lowering effect ${ }^{(26)}$. The analysis in the latter review revealed a $7.4 \%$ reduction in LDL-cholesterol with a mean nut consumption of $67 \mathrm{~g} / \mathrm{d}$. Reductions in
LDL-cholesterol were dose dependent, but not dependent on the type of nut consumed ${ }^{(26)}$. The lipid-lowering effects may be attributed to the high content of unsaturated fat and fibre in nuts. Other bioactive nutrients in nuts may benefit glucoregulation ${ }^{(27)}$, endothelial function, blood pressure control $^{(28)}$ and inflammation ${ }^{(21)}$. Studies have demonstrated that higher nut consumers are at a significantly lower risk of non-cardiovascular inflammatory disease mortality ${ }^{(29)}$ and risk of developing type 2 diabetes $^{(30)}$ than low nut consumers. These benefits may be attributed to their nutrient profile; plant-derived $n-3$ fatty acids ( $\alpha$-linolenic acid; ALA) found in walnuts have been shown in clinical and epidemiological studies to improve inflammation, arterial compliance, insulin resistance, endothelial function and blood pressure ${ }^{(31-34)}$. Nuts, especially consumed with their skin intact, have a significant amount of polyphenols ${ }^{(35)}$. The results of many epidemiological studies suggest that the intake of polyphenol-rich foods has a beneficial effect on a large number of cardiovascular risk factors including high blood pressure and poor vascular function ${ }^{(36)}$. Polyphenols and vitamin $\mathrm{E}$ may have a role in modifying some of the inflammatory mediators $^{(37,38)}$ and be beneficial for cognitive performance $^{(39,40)}$. Unsalted nuts contain high levels of $\mathrm{K}$ and $\mathrm{Mg}$, making them a potential food for blood pressure control. However, nuts are commonly sold as a highly salted product and in this form can substantially increase the intake of $\mathrm{Na}$, hence reducing their potential benefit. In addition, nuts contain fibre and L-arginine that has been shown to improve endothelial function ${ }^{(41-44)}$. Studies have investigated the impact of nuts on endothelial function $^{(28)}$; however, no study has taken the next step and considered whether nuts may have beneficial effects on

Table 1. Nutritional composition of nuts (per $100 \mathrm{~g}$ )

\begin{tabular}{|c|c|c|c|c|c|c|c|c|c|}
\hline Nutrient & Almonds & Brazils & Cashews & Hazelnuts & Macadamias & Pecans & Groundnuts & Pistachios & Walnuts \\
\hline Energy (kJ)* & 2432 & 2755 & 2323 & 2639 & 3015 & 2902 & 2381 & 2360 & 2747 \\
\hline $\operatorname{SFA}(g)^{*}$ & 4 & 15 & 8 & 4 & 12 & 7 & 7 & 6 & 6 \\
\hline $\operatorname{MUFA}(g)^{*}$ & 32 & 25 & 24 & 46 & 59 & 40 & 24 & 24 & 9 \\
\hline PUFA $(g)^{*}$ & 12 & 21 & 8 & 8 & 2 & 21 & 16 & 14 & 47 \\
\hline$\alpha$-Linolenic acid $(\mathrm{g})^{*}$ & 0.0 & $0 \cdot 2$ & 0.2 & 0.1 & 0.1 & 0.6 & 0.0 & $0 \cdot 0$ & $6 \cdot 0$ \\
\hline Protein $(g)^{\star}$ & 21 & 14 & 18 & 17 & 8 & 9 & 25 & 20 & 15 \\
\hline Arginine $(g)^{*}$ & $2 \cdot 5$ & $2 \cdot 2$ & $2 \cdot 0$ & $2 \cdot 2$ & $1 \cdot 2$ & $3 \cdot 0$ & $1 \cdot 2$ & $2 \cdot 2$ & $2 \cdot 3$ \\
\hline Fibre $(g)^{\star}$ & 13 & 9 & 3 & 10 & 9 & 10 & 9 & 10 & 7 \\
\hline Total vitamin $\mathrm{E}(\mathrm{mg}) \dagger$ & 27 & 4 & 1 & 33 & 1 & 4 & 8 & 7 & 6 \\
\hline $\mathrm{Na}(\mathrm{mg})^{*}$ & 1 & 3 & 12 & 0 & 5 & 0 & 18 & 1 & 2 \\
\hline $\mathrm{K}(\mathrm{mg})^{*}$ & 733 & 659 & 660 & 680 & 368 & 410 & 705 & 1025 & 441 \\
\hline $\operatorname{Mg}(\mathrm{mg})^{*}$ & 270 & 376 & 292 & 163 & 130 & 121 & 168 & 121 & 158 \\
\hline Anthocyanins (mg) & 184 & 0 & 9 & 501 & 0 & 494 & 16 & 237 & 67 \\
\hline Flavonoids $(\mathrm{mg}) \S$ & 40 & 29 & 42 & 14 & 9 & 639 & 146 & 87 & 535 \\
\hline Resveratrol $(\mu \mathrm{g}) \|$ & $\mathrm{N} / \mathrm{A}$ & $\mathrm{N} / \mathrm{A}$ & $\mathrm{N} / \mathrm{A}$ & $\mathrm{N} / \mathrm{A}$ & $\mathrm{N} / \mathrm{A}$ & $\mathrm{N} / \mathrm{A}$ & 102 & 117 & $\mathrm{~N} / \mathrm{A}$ \\
\hline $\begin{array}{l}\text { Total antioxidant } \\
\text { content (with pellicle) }\end{array}$ & 0.41 & 0.25 & 0.39 & 0.71 & 0.42 & $8 \cdot 3$ & $2 \cdot 0$ & 1.3 & $23 \cdot 1$ \\
\hline
\end{tabular}

N/A, not available.

* Data from US Department of Agriculture, Agricultural Research Service ${ }^{(140)}$

†Data from Kornsteiner et al. ${ }^{(141)}$

$\ddagger$ Data from Bolling et al. ${ }^{(35)}$.

$\S$ Data from Yang et al. ${ }^{(142)}$.

|| Data from Tokuşoglu et al. ${ }^{(143)}$.

I Data from Blomhoff et al. ${ }^{(144)}$ 
cerebral vascular function and little research has been conducted on the impact of nut consumption on cognitive performance.

Thus, unsalted nuts contain the precursor, key ingredients for cardiometabolic benefits needed to enhance blood vessel health, which may in turn improve cognitive function and limit cognitive decline as proposed in Fig. 1. Using a systematic search protocol, we reviewed the evidence for the effects of both tree and ground nuts on glucoregulation, blood pressure, arterial compliance, inflammation, endothelial vasodilator function and cognitive performance. As noted previously, there is a large body of consistent evidence demonstrating improvements in lipid regulation with nut consumption ${ }^{(25,26)}$; hence this component has not been included in the present review.

\section{Methods}

\section{Selection of studies}

Medline (via Ovid) and CINAHL (via Ebsco host) databases and the Cochrane Library were searched on 21 November 2012. Search terms used included MeSH (Medical Subject
Headings) terms: 'nuts' OR 'almond*' OR 'Brazil nut*' OR 'cashew*' OR 'hazelnut', OR 'macadamia*' OR 'peanut*' OR 'pecan*' OR 'pistachio*' OR 'walnut*' AND 'endothel*' OR 'FMD' OR 'vascular*' OR 'blood pressure' OR 'arterial compliance' OR 'vasodilatation' OR 'glucose' OR 'insulin' OR 'inflam*' OR 'cognit*'. Limits included 'human only' and 'English language'. In addition, reference lists from the publications identified by the database searches were also manually searched to identify other relevant articles that were not detected by the searches. Studies were included if they met the following criteria: intervention or epidemiological studies in human subjects. Intervention diets included at least one of the following nuts: almonds, cashews, hazelnuts, macadamias, groundnuts, pistachios, walnuts, pecans or Brazil nuts. Intervention studies included assessment of chronic nut consumption for a minimum period of 3 weeks, thereby assessing chronic changes. Published studies were required to be original research and evaluate the effects of nuts on at least one of the following in human subjects: glucoregulation, endothelial vasodilator function, arterial compliance, resting blood pressure, inflammation or cognitive performance.

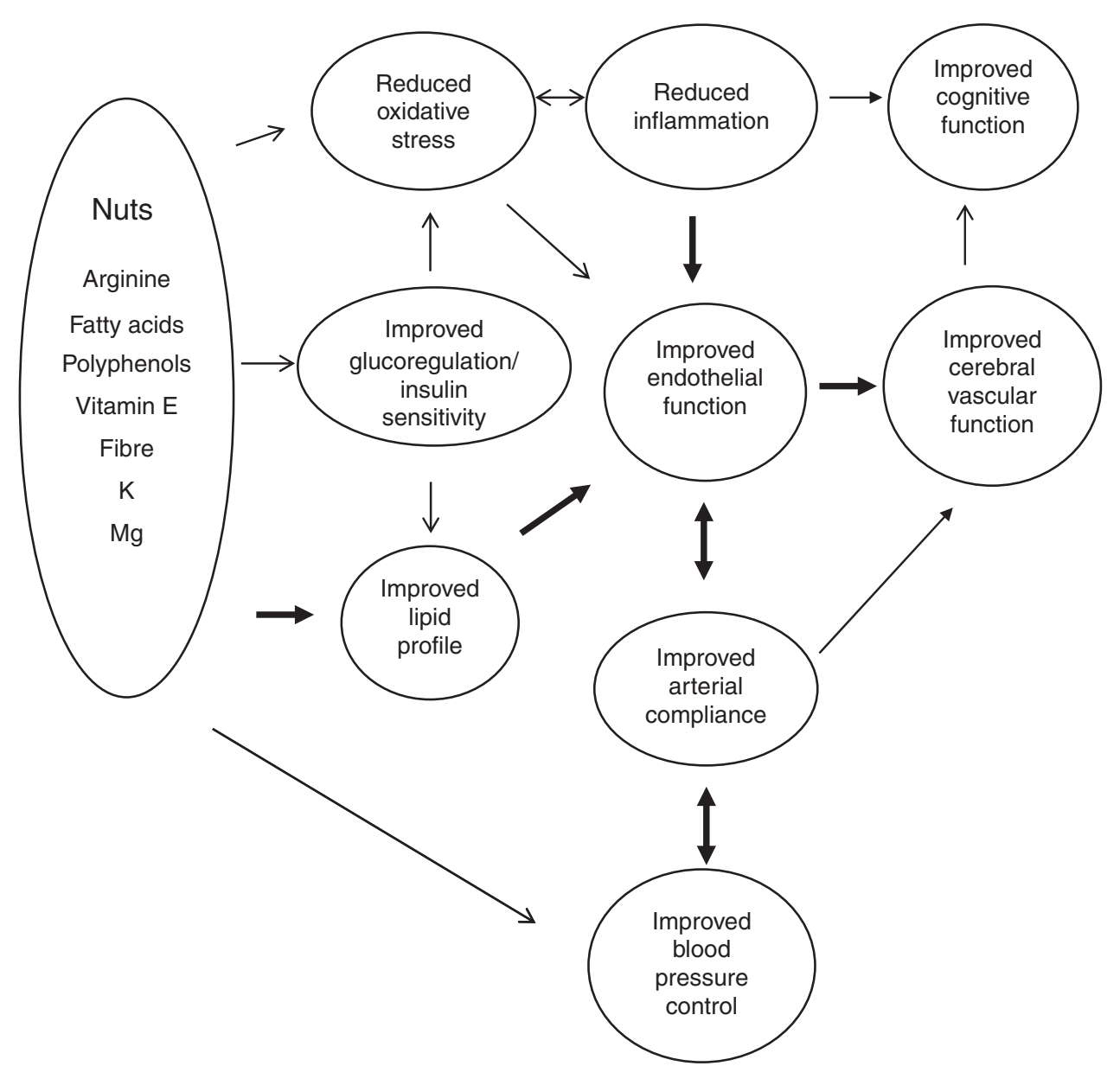

Fig. 1. Summary of potential effects of nutrients in nuts to improve cardiovascular risk factors (lipid profile, arterial compliance, glucoregulation, oxidative stress, blood pressure and inflammation) and consequent improvement in endothelial function and potential improvement in cerebral vascular function and hence cognitive performance. $\rightarrow$, Weak evidence; $\rightarrow$, strong evidence; $\leftrightarrow$, bi-directional effect; $\leftrightarrow$, strong bi-directional effect. 
Studies were excluded if they were non-English-language papers, narrative reviews, systematic reviews, expert opinions, editorials, abstracts, letters to the editor, theses, or animal or in vitro studies. Weighted mean changes in glucoregulation, systolic and diastolic blood pressure, CRP, ICAM-1, VCAM-1 and endothelial vasodilator function were calculated for studies that reported data suitable for calculating a percentage change. Study sample size was used to weight the calculation of the overall mean percentage change across studies using STATA software (StataIC 11; StataCorp LP).

\section{Results}

The search revealed articles published between March 1993 and October 2013. Of the 4198 articles identified by all databases and nine articles identified from hand searching, 3019 were excluded as duplicates, 114 were excluded because of document type (review, note, letter, proceedings paper, or meeting abstract) and 837 were excluded because they did not assess endothelial function, blood pressure, inflammation, glucoregulation or cognitive performance in conjunction with nut consumption. Of the 237 articles screened (titles and abstracts), 166 were excluded because they did not meet the inclusion criteria. Therefore, seventy-one studies were included in the present review as shown in the PRISMA (Preferred Reporting Items for Systematic Reviews and Meta-Analyses) flowchart (Fig. 2). A total of forty-four studies evaluated blood pressure, thirty-two evaluated glucoregulation, thirty-one evaluated inflammatory markers, nine evaluated endothelial vasodilator function, two evaluated arterial compliance and four evaluated cognitive performance. A total of nine types of nut were used in these studies: almonds, Brazil nuts, cashews, hazelnuts, macadamias, groundnuts, pistachios, pecans and walnuts. The majority of studies examined walnuts, almonds and mixed/any nuts (Table 2).

Most research measuring the effect of nut consumption on glucoregulation, blood pressure, inflammation, arterial compliance, endothelial vasodilator function and cognition has been performed with walnuts, mixed or non-specified nuts, almonds and pistachios, with only seven studies using groundnuts, hazelnuts, cashews, Brazil nuts or macadamias and no studies with pecans (Table 2). Studies are summarised in Tables 3-8 and are grouped according to outcomes, presented in order of efficacy (using mean percentage or blood pressure $(\mathrm{mmHg}$ ) change where available). The following information was also extracted: author and year of publication, number, age and sex of the participants, type of individuals studied (i.e. healthy, hyperlipidaemic, high $\mathrm{CHD}$ risk, type 2 diabetes, overweight/obese or metabolic syndrome), study design,

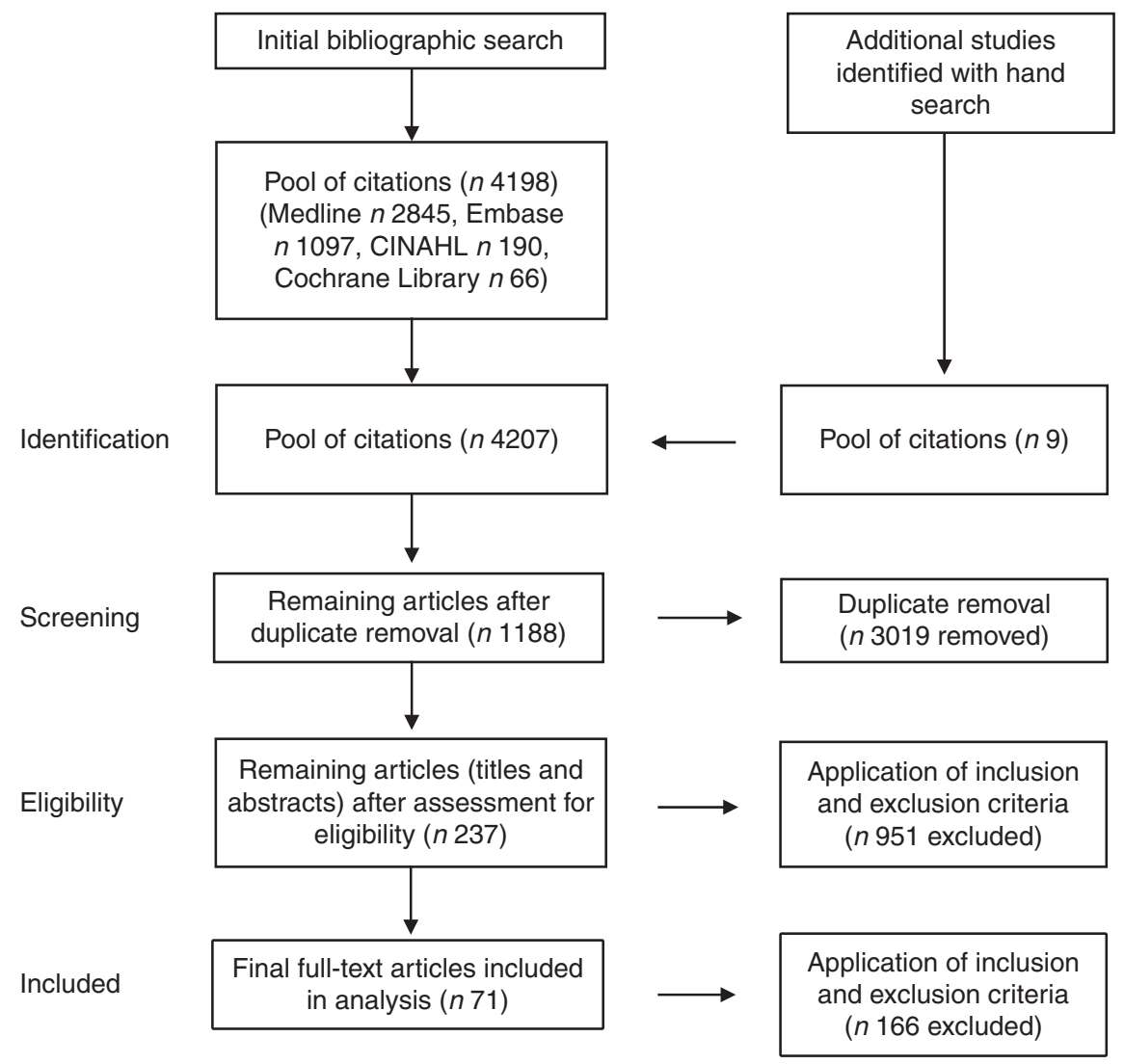

Fig. 2. PRISMA (Preferred Reporting Items for Systematic Reviews and Meta-Analyses) flow chart showing procedures used to identify studies investigating the effect of nuts on blood pressure, endothelial function, inflammation, arterial compliance, glucoregulation and cognition included in the systematic search. 
Table 2. Number of measures of nut consumption on the effect on blood pressure, glucoregulation, inflammation, arterial compliance, endothelial function and cognition (some studies tested more than one type of nut)

\begin{tabular}{lcccccc}
\hline Nut type & Blood pressure & Glucoregulation & Inflammation & $\begin{array}{c}\text { Arterial } \\
\text { compliance }\end{array}$ & $\begin{array}{c}\text { Endothelial } \\
\text { function* }\end{array}$ & Cognition \\
\hline Walnut & 11 & 9 & 9 & 1 & 4 & 2 \\
Mixed/any nut & 14 & 12 & 10 & 1 & 2 & 2 \\
Almond & 10 & 8 & 8 & - & - & - \\
Pistachio & 3 & 2 & 1 & - & 2 & - \\
Hazelnut & 1 & 1 & 2 & - & 1 & - \\
Cashew & 1 & - & 1 & - & - & - \\
Brazil & - & - & - & - & - & - \\
Macadamia & 1 & 1 & - & - & - & - \\
Groundnut & 1 & - & - & - & - & 4 \\
Pecan & - & 34 & 31 & 2 & 9 & 4 \\
Total & 42 & & 1 & & - & - \\
\hline
\end{tabular}

*Endothelial vasodilator function assessed by either flow-mediated dilatation or Endo-PAT device.

length of intervention, type and dose of nut, controls used and effect-size calculations where possible. Fig. 3 presents the number of outcome measures and the type of studies reflecting the level of evidence for these studies according to National Health and Medical Research Council guidelines ${ }^{(45)}$. Most intervention studies were randomised and controlled, providing greater evidence than uncontrolled or non-randomised trials.

\section{Effects of nuts on glucoregulation}

Details of studies measuring the effect of nut consumption on glucoregulation are reported in Table 3. A total of eight observational and twenty-four intervention trials evaluated the effects of chronic consumption of nuts on glucoregulation. Nuts consumed included walnuts, pistachios, groundnuts, almonds, cashews and mixed nuts, with amounts consumed ranging from 10 to $108 \mathrm{~g} / \mathrm{d}$ ( $\frac{1}{3}$ ounce to 4 ounces) (approximately $2-20 \%$ of energy intake). The duration of consumption ranged from 4 weeks to 16 years. Intervention studies made comparisons with a healthy diet (fourteen studies), habitual diet (three studies), high-fat diet (one study) or other food products (five studies): muffins, pretzels, cereal bar, cheese or another type of nut. One study used no control. Of the studies, four compared habitual or healthy diets with intervention diets including nuts (NORDIET ${ }^{(46)}$ or a Mediterranean $\left.\operatorname{diet}^{(47,48)}\right)$.

Tree nuts were associated with a lower prevalence of fasting hyperglycaemia compared with non-nut consumers in the National Health and Nutrition Survey (NHANES) cohort study ${ }^{(49)}$. However, a healthy dietary pattern including nuts found no association with fasting glucose or insulin $^{(50)}$. It is possible that the amount of nuts consumed was insufficient to show benefits. Nut consumption has also been associated with a reduced risk of type 2 diabetes; evidence to support this comes from large epidemiological studies $^{(51-54)}$. The Nurses' Health Study demonstrated that consumption of nuts ( $\geq 5$ times per week), peanut butter ( $\geq 5$ times per week) or walnuts ( $\geq$ twice per week) was associated with a 24,21 and $15 \%$ lower risk, respectively, of developing type 2 diabetes ${ }^{(51,54)}$ compared with those who never or rarely ate nuts; the effect was greatest in those of healthy body weight ${ }^{(51)}$. In addition, the Shanghai Women's Health Study demonstrated that groundnut consumption was associated with a $22 \%$ decreased risk of type 2 diabetes ${ }^{(55)}$. The SUN Study demonstrated a $35 \%$ reduced risk of type 2 diabetes with a Mediterranean diet including an unspecified quantity of nuts ${ }^{(53)}$. However, other components of the Mediterranean diet including olive oil and a high fibre intake may have also contributed to this outcome ${ }^{(56)}$. In contrast, the Iowa Women's Health Study did not find any association of consumption of foods high in vegetable fat (including nuts) and incidence of type 2 diabetes ${ }^{(57)}$, which may in part be due to the low mean intake of nuts in this cohort.

Clinical trials examining nut consumption and diabetes risk, glycaemic control or insulin resistance have suggested some beneficial effects. Some short-term intervention studies have shown benefits of nut consumption on glucose homeostasis ${ }^{(58,59)}$ and insulin secretion ${ }^{(46,58,60,61)}$. The effects of nuts on insulin sensitivity are influenced strongly by changes in body weight, which may have accounted for the changes observed in one of these studies where participants reduced body weight with nut consumption. Longer intervention trials with Mediterranean diets supplemented daily with $20-50 \mathrm{~g}$ of walnuts or $30 \mathrm{~g}$ of mixed nuts (a mixture of walnuts, almonds and hazelnuts was used in the PREvencion con DIeta MEDiterranea (PREDIMED) trial as reported by Casas-Agustench et al. ${ }^{(21)}$ ) resulted in a reduction in fasting glucose, insulin and improvement in insulin sensitivity (homeostatic model assessment of insulin resistance; HOMA) ${ }^{(47)}$ and the incidence of type 2 diabetes by $52 \%$ over 4 years ${ }^{(30)}$. Benefits shown in studies with nuts included as part of the intervention diet (NORDIET ${ }^{(46)}$ or Mediterranean $\operatorname{diet}^{(47)}$ ) may have been partly due to other components of these diets ${ }^{(62)}$. Other studies have not shown benefits; consumption of pistachios, almonds, walnuts and a Mediterranean diet (supplemented with $10 \mathrm{~g}$ nuts/d) revealed no effect on 
Table 3. Studies measuring effect of nut consumption on glucoregulation

\begin{tabular}{|c|c|c|c|c|c|c|c|}
\hline Author & Time & Participants & Study design & Amount/type of nuts & \multicolumn{2}{|c|}{ Outcomes† } & $\begin{array}{l}\text { Effect; } \\
\text { effect size }\end{array}$ \\
\hline \multicolumn{8}{|c|}{ Observational studies measuring effect of nut consumption on diabetes risk and elevated glucose (studies presented in order of efficacy) } \\
\hline $\begin{array}{l}\text { Martínez-González } \\
\text { et al. }(2008)^{(53)}\end{array}$ & 4 years & $\begin{array}{l}n 13380, \text { healthy, } M \text { and } F \\
\text { mean } 38 \text { (SD 12) years }\end{array}$ & $\begin{array}{l}\text { Prospective cohort } \\
\text { (SUN study), FFQ } \\
\text { and incidence of Dm }\end{array}$ & $\begin{array}{l}\text { Tertiles of Med diet } \\
\text { (unspecified amount } \\
\text { of nuts) }\end{array}$ & $\begin{array}{l}\text { RR } \\
1.0 \\
0.41(95 \% \mathrm{Cl} 0.2,0.9) \\
0.17(95 \% \mathrm{Cl} 0.04,0.8)\end{array}$ & $\begin{array}{l}\text { Med diet } \downarrow 35 \% \mathrm{RR} \\
\text { incidence of } \mathrm{Dm}\end{array}$ & + \\
\hline $\begin{array}{l}\text { Jiang et al. } \\
(2002)^{(51)}\end{array}$ & 16 years & $\begin{array}{l}n 137856, \text { healthy, } F \\
\text { mean } 46 \text { (range } 34-59) \\
\text { years }\end{array}$ & $\begin{array}{l}\text { Prospective cohort } \\
\text { (Nurses' Health Study), } \\
\text { FFQ and incidence of } \\
\text { Dm }\end{array}$ & $\begin{array}{l}\text { Quantiles of nuts/peanut } \\
\text { butter } \\
\text { Never/rare } \\
<1 \text { time/week } \\
1-4 \text { times/week } \\
>5 \text { times/week }\end{array}$ & $\begin{array}{l}\mathrm{RR} \\
1.0 \\
0.98(95 \% \mathrm{Cl} 0.9,1 \cdot 1) \\
0.91(95 \% \mathrm{Cl} 0.8,1.0) \\
0.79(95 \% \mathrm{Cl} 0.7,0.8)\end{array}$ & $\begin{array}{l}\text { Nuts } \downarrow 24 \% \text { RR, peanut } \\
\text { butter } \downarrow 21 \% \text { RR } \\
\text { incidence of Dm }\end{array}$ & + \\
\hline $\begin{array}{l}\text { Villegas et al. } \\
(2008)^{(55)}\end{array}$ & 5 years & $\begin{array}{r}n 64227, \text { healthy, } F \text {, mean } \\
49 \text { (range } 43-63 \text { ) years }\end{array}$ & $\begin{array}{l}\text { Prospective cohort } \\
\text { (Shanghai Women's } \\
\text { Health Study), FFQ and } \\
\text { incidence of Dm }\end{array}$ & $\begin{array}{l}\text { Quintiles of groundnut } \\
\text { consumption }\end{array}$ & $\begin{array}{l}\text { RR } \\
1.0 \\
0.8(95 \% \mathrm{Cl} 0.7,0.9) \\
0.95(95 \% \mathrm{Cl} 0.82,1.1) \\
0.79(95 \% \mathrm{Cl} 0.7,0.9) \\
0.8(95 \% \mathrm{Cl} 0.7,0.9)\end{array}$ & $\begin{array}{l}\text { Groundnuts } \downarrow 20 \% \text { RR } \\
\text { incidence of } \mathrm{Dm}\end{array}$ & + \\
\hline $\begin{array}{l}\text { Pan et al. } \\
(2013)^{(54)}\end{array}$ & 4 years & $\begin{array}{l}n 137856, \text { healthy, } \mathrm{F}, \\
\quad \text { mean } 52(\mathrm{SD} 10) \text { years }\end{array}$ & $\begin{array}{l}\text { Prospective cohort } \\
\text { (Nurses' Health Study } \\
\text { cohorts } 1 \text { and 2), FFQ } \\
\text { and incidence of Dm }\end{array}$ & $\begin{array}{l}\text { Quintiles of walnut } \\
\text { consumption } \\
\text { Never/rare } \\
<1 \text { serve/week } \\
1 \text { serve/week } \\
\geq 2 \text { serves/week }\end{array}$ & $\begin{array}{l}\text { RR } \\
1.0 \\
1.01(95 \% \mathrm{Cl} 0.95,1.08) \\
1.01(95 \% \mathrm{Cl} 0.90,1.13) \\
1.04(95 \% \mathrm{Cl} 0.92,1.18)\end{array}$ & $\begin{array}{c}\text { Walnuts } \downarrow 15 \% \mathrm{RR} \\
\text { incidence of } \mathrm{Dm}\end{array}$ & + \\
\hline $\begin{array}{l}\text { Meyer et al. } \\
(2001)^{(57)}\end{array}$ & 11 years & $\begin{array}{l}n 7210, \text { high risk of CVD, } \\
M \text { and } F, \text { mean } 68 \\
(S D 6) \text { years }\end{array}$ & $\begin{array}{l}\text { X-sect, FFQ, prevalence } \\
\text { of elevated glucose } \\
\text { (PREDIMED) }\end{array}$ & $\begin{array}{l}\text { Quintiles of vegetable fat } \\
\text { (including nuts) }\end{array}$ & Data N/A & $\begin{array}{l}\text { No association of } \\
\text { incidence of Dm with } \\
\text { nut consumption }\end{array}$ & NS-G \\
\hline \multicolumn{8}{|c|}{ Cross-sectional studies measuring effect of nut consumption on diabetes risk and elevated glucose (studies presented in order of efficacy) } \\
\hline $\begin{array}{l}\text { Nettleton et al. } \\
(2008)^{(145)}\end{array}$ & $X$-sect & $\begin{array}{l}n 5011 \text {, healthy, } M \text { and } \\
F, 45-84 \text { years }\end{array}$ & $\begin{array}{l}\mathrm{X} \text {-sect, MESA, FFQ and } \\
\text { prevalence of Dm }\end{array}$ & $\begin{array}{l}\text { Quintiles of healthy dietary } \\
\text { pattern (including any } \\
\text { nuts) }\end{array}$ & & $\begin{array}{l}\downarrow 15 \% \text { RR incidence } \\
\text { of Dm with nut } \\
\text { consumption }\end{array}$ & + \\
\hline \multirow[t]{2}{*}{$\begin{array}{l}\text { O'Neil et al. } \\
(2011)^{(49)}\end{array}$} & $X$-sect & $\begin{array}{l}n 13292 \text {, general } \\
\text { population, } M \text { and } \\
F, 19-50+\text { years }\end{array}$ & $\begin{array}{l}\text { X-sect, } 1999-2004 \\
\text { NHANES, } 24 \mathrm{~h} \text { recall } \\
\text { and prevalence of } \\
\text { elevated glucose }\end{array}$ & 'All' nut group $\geq 7 \mathrm{~g} / \mathrm{d}(\mathrm{A})$ & & $\begin{array}{l}\text { No association of } \\
\text { prevalence of elevated } \\
\text { glucose with 'all' } \\
\text { nut consumption }\end{array}$ & NS-G \\
\hline & & & & Tree nut group $\geq 7$ g/d (B) & & $\begin{array}{l}4 \% \downarrow \text { prevalence } \\
\text { elevated glucose with } \\
\text { tree nut consumption }\end{array}$ & + \\
\hline $\begin{array}{l}\text { Ibarrola-Jurado } \\
\text { et al. }(2013)^{(81)}\end{array}$ & $X$-sect & $\begin{array}{l}n 7210, \text { high risk of CVD, } \\
M \text { and } F, \text { mean } 68 \\
(S D 6) \text { years }\end{array}$ & $\begin{array}{l}\text { X-sect, FFQ, prevalence } \\
\text { of elevated glucose } \\
\text { (PREDIMED) }\end{array}$ & $\begin{array}{l}\text { Tertiles (any nuts) } \\
<28 \mathrm{~g} / \mathrm{week} \\
28-84 \mathrm{~g} / \mathrm{week} \\
>84 \mathrm{~g} / \text { week }\end{array}$ & $\begin{array}{l}1.0 \\
0.95(95 \% \mathrm{Cl} 0.71,1.29) \\
0.85(95 \% \mathrm{Cl} 8.81,1.53)\end{array}$ & $\begin{array}{l}\text { No association of elevated } \\
\text { glucose with nut } \\
\text { consumption }\end{array}$ & NS-G \\
\hline \multicolumn{8}{|c|}{ Intervention studies measuring effect of nut consumption on glucoregulation (studies presented in order of efficacy) } \\
\hline $\begin{array}{l}\text { Salas-Salvadó } \\
\text { et al. }(2011)^{(30)}\end{array}$ & 4 years & $\begin{array}{l}n 418 \text { (control }=134, \\
\text { nuts }=145, \mathrm{OO}=139) \\
\text { high risk of } \mathrm{CVD}, \mathrm{M} \text { and } \\
\mathrm{F}, \text { mean } 68 \text { (range } \\
55-80 \text { ) years }\end{array}$ & $\begin{array}{l}\text { RCT, parallel, Med diet + } \\
\text { OO v. Med diet + nuts } \\
\text { v. LF diet (control) } \\
\text { (PREDIMED) }\end{array}$ & $30 \mathrm{~g} / \mathrm{d}$ mixed nuts $\ddagger$ & $\begin{array}{l}\downarrow 52 \% \text { RR incidence } \\
\text { of Dm }\end{array}$ & & + \\
\hline
\end{tabular}




\section{䯽}

Table 3. Continued

\begin{tabular}{|c|c|c|c|c|c|c|}
\hline Author & Time & Participants & Study design & Amount/type of nuts & Outcomes† & $\begin{array}{l}\text { Effect; } \\
\text { effect size }\end{array}$ \\
\hline $\begin{array}{l}\text { Esposito et al. } \\
(2004)^{(47)}\end{array}$ & 2 years & $\begin{array}{l}n 180 \text { (control = 90, Med } \\
\text { diet }=90), \text { Met-S, M } \\
\text { and F, mean } 44 \text { (SD 6) } \\
\text { years }\end{array}$ & $\begin{array}{l}\text { RCT, parallel, Med diet } \\
\text { (including nuts) } v \text {. } \\
\text { prudent diet (control) }\end{array}$ & $20-50 \mathrm{~g} / \mathrm{d}$ walnuts & $\begin{array}{l}\text { Glucose } \downarrow 5 \%^{*} \\
\text { Insulin } \downarrow 33 \%^{*} \\
\text { HOMA } \downarrow 45 \%^{*}\end{array}$ & $\begin{array}{l}+; 0.8 \\
+; 0.8 \\
+; 0.8\end{array}$ \\
\hline $\begin{array}{l}\text { Wien et al. } \\
(2010)^{(61)}\end{array}$ & 16 weeks & $\begin{array}{l}n 65 \text { (control }=32, \text { nut } \\
=33 \text { ), pre-diabetes, M } \\
\text { and } \mathrm{F}, \text { mean } 53(\mathrm{SD} 9) \\
\text { years }\end{array}$ & $\begin{array}{l}\text { RCT, parallel, American } \\
\text { Diabetes Association } \\
\text { diet (control) with/ } \\
\text { without almonds }\end{array}$ & $56 \mathrm{~g} / \mathrm{d}$ almonds & $\begin{array}{l}\text { Glucose NS } \\
\text { Insulin } \downarrow 32 \% \%^{*} \\
\text { HOMA } \downarrow 40 \% * \\
\text { HbA1c NS }\end{array}$ & $\begin{array}{l}\text { NS-G; } 0.2 \\
+; 1.3 \\
+; 0.2 \\
\text { NS-G; } 0.4\end{array}$ \\
\hline $\begin{array}{l}\text { Casas-Agustench } \\
\text { et al. }(2011)^{(60)}\end{array}$ & 12 weeks & $\begin{array}{l}n 50 \text { (control }=25, \text { nut } \\
=25), \text { Met-S, M and F, } \\
\text { mean } 52(\mathrm{SD} 8 \text { ) years }\end{array}$ & $\begin{array}{l}\text { RCT, parallel, isoenergetic } \\
\text { healthy diet (control) } \\
\text { with/without nuts }\end{array}$ & $30 \mathrm{~g} / \mathrm{d}$ mixed nuts $\ddagger$ & $\begin{array}{l}\text { Glucose NS } \\
\text { Insulin } \downarrow 33 \%^{*} \\
\text { HOMA } \downarrow 33 \%^{*}\end{array}$ & $\begin{array}{l}\text { NS-G; } 0.0 \\
+; 0.4 \\
+; 0.4\end{array}$ \\
\hline $\begin{array}{l}\text { Adamsson et al. } \\
(2011)^{(46)}\end{array}$ & 4 weeks & $\begin{array}{l}n 86, \mathrm{M} \text { and } \mathrm{F} \text { (control } \\
=42, \mathrm{NORDIET}=44) \\
\text { mean } 53 \text { (SD } 8 \text { ) years, } \\
\text { hypercholesterolaemic }\end{array}$ & $\begin{array}{l}\text { RCT parallel, NORDIET } \\
\text { (high fibre, fish, LF } \\
\text { dairy, nuts) } v \text {. habitual } \\
\text { diet (control) }\end{array}$ & Ad libitum almonds & $\begin{array}{l}\text { Glucose NS } \\
\text { Insulin } \downarrow 24 \%^{*} \\
\text { HOMA } \downarrow 25 \%^{*}\end{array}$ & $\begin{array}{l}\text { NS-G; } 0.0 \\
+; 0.5 \\
+; 0.6\end{array}$ \\
\hline $\begin{array}{l}\text { Sari et al. } \\
(2010)^{(59)}\end{array}$ & $\begin{array}{l}4 \text { weeks } \\
\text { per arm }\end{array}$ & $\begin{array}{l}n 32, \text { healthy } M, \text { mean } 22 \\
\text { (range } 21-24 \text { ) years }\end{array}$ & $\begin{array}{l}\text { Prospective cohort, } \\
\text { isoenergetic Med diet } \\
\text { (control) with/without } \\
\text { pistachios, no washout }\end{array}$ & $80-100 \mathrm{~g} / \mathrm{d}$ pistachios & Glucose $\downarrow 9 \%$ & $+; 0.9$ \\
\hline $\begin{array}{l}\text { Kalgaonkar et al. } \\
(2011)^{(62)}\end{array}$ & 6 weeks & $\begin{array}{l}n 31 \text { (almond }=14, \\
\text { walnut }=17), \mathrm{F}, \text { with } \\
\text { PCOS, age range } \\
20-45 \text { years }\end{array}$ & $\begin{array}{l}\text { Pre-/post-measures } \\
\text { walnuts } v \text {. almonds }\end{array}$ & $\begin{array}{l}36 \mathrm{~g} / \mathrm{d} \text { walnuts }(\mathrm{W}) \\
26 \mathrm{~g} / \mathrm{d} \text { almonds }(\mathrm{A})\end{array}$ & $\begin{array}{l}\text { Glucose NS (W v. A) } \\
\text { Insulin NS (W v. A) } \\
\text { HOMA NS (W v. A) } \\
\text { HbA1c } \downarrow 4 \%^{*}(W v . A)\end{array}$ & $\begin{array}{l}\text { NS-G } \\
\text { NS-G; } 0.8 \\
\text { NS-G; } 2.3 \\
+; 0.1\end{array}$ \\
\hline $\begin{array}{l}\text { Estruch et al. } \\
(2006)^{(58)}\end{array}$ & 12 weeks & $\begin{array}{l}n 772 \text { (control }=257, \mathrm{OO} \\
=257, \text { nuts }=258), \\
\text { high risk of CVD, M and } \\
F, \text { mean } 69(\mathrm{SD} 6 \text { ) years }\end{array}$ & $\begin{array}{l}\text { RCT, parallel, Med diet + } \\
\text { OO v. Med diet + nuts } \\
\text { v. LF diet (control) } \\
\text { (PREDIMED) }\end{array}$ & $30 \mathrm{~g} / \mathrm{d}$ mixed nuts $\ddagger$ & $\begin{array}{l}\text { Glucose } \downarrow^{*} \text { data N/A } \\
\text { Insulin } \downarrow^{*} \text { data N/A } \\
\text { HOMA } \downarrow^{*} \text { data N/A }\end{array}$ & $\begin{array}{l}+; 0.1 \\
+; 0.2 \\
+; 0.3\end{array}$ \\
\hline $\begin{array}{l}\text { Cohen \& Johnston } \\
(2011)^{(64)}\end{array}$ & 12 weeks & $\begin{array}{l}n 13 \text { (control = 6, nut } \\
\quad 6) \text {, Dm, mean } 66 \\
\text { (SD 3) years }\end{array}$ & $\begin{array}{l}\text { Pilot study, RCT, parallel } \\
\text { almonds or cheese } \\
\text { (control) }\end{array}$ & $28 \mathrm{~g} / \mathrm{d}$ almonds & $\begin{array}{l}\text { Glucose NS } \\
\text { Insulin NS } \\
\text { HbA1c } \downarrow 4 \%^{*}\end{array}$ & $\begin{array}{l}\text { NS-G; } 0.3 \\
\text { NS-G; } 0.7 \\
+; 1.5\end{array}$ \\
\hline \multirow[t]{2}{*}{$\begin{array}{l}\text { Lovejoy et al. } \\
(2002)^{(65)}\end{array}$} & $\begin{array}{l}4 \text { weeks } \\
\text { per arm }\end{array}$ & $\begin{array}{l}\text { Study } 1, n 20 \text {, healthy, } \\
\text { mean } 25 \text { (SD 1) years }\end{array}$ & $\begin{array}{l}\text { Prospective cohort (no } \\
\text { control) habitual diet }+ \\
\text { almonds }\end{array}$ & $100 \mathrm{~g} / \mathrm{d}$ almonds & $\begin{array}{l}\text { Glucose NS } \\
\text { Insulin NS }\end{array}$ & $\begin{array}{l}\text { NS-G; } 0.2 \\
\text { NS-G; } 0.1\end{array}$ \\
\hline & & $\begin{array}{l}\text { Study } 2, n 30, \mathrm{Dm} \text {, mean } \\
\quad 54 \text { (SD 2) years }\end{array}$ & $\begin{array}{l}\text { RCT, cross-over, HF } \\
\text { (control) with/without } \\
\text { almonds and LF } \\
\text { (control) with/without } \\
\text { almonds }\end{array}$ & $\begin{array}{l}57-113 \mathrm{~g} / \mathrm{d}(10 \% \\
\text { energy) almonds }\end{array}$ & $\begin{array}{l}\text { HF glucose NS } \\
\text { LF glucose NS } \\
\text { HF insulin NS } \\
\text { LF insulin NS } \\
\text { HF HbA1c NS } \\
\text { LF HbA1c NS }\end{array}$ & $\begin{array}{l}\text { NS-G; } 0 \cdot 3 \\
\text { NS-G; } 0 \cdot 2 \\
\text { NS-G } \\
\text { NS-G } \\
\text { NS-G } \\
\text { NS-G }\end{array}$ \\
\hline $\begin{array}{l}\text { Jenkins et al. } \\
(2011)^{(66)}\end{array}$ & 3 months & $\begin{array}{l}n 117 \text { (control }=37, \\
37 \mathrm{~g} / \mathrm{d} \text { nut }=40,75 \mathrm{~g} / \mathrm{d} \\
\text { nut }=40), \mathrm{Dm}, \mathrm{M} \text { and } \\
\mathrm{F}, \text { mean } 62(\mathrm{sD} 10) \\
\text { years }\end{array}$ & $\begin{array}{l}\text { RCT, parallel, } 75 \mathrm{~g} / \mathrm{d} \text { nut } v \text {. } \\
37 \mathrm{~g} / \mathrm{d} \text { nut }+ \text { half-dose } \\
\text { muffin } v \text {. muffin (control) }\end{array}$ & $\begin{array}{l}75 \text { or } 37 \mathrm{~g} / \mathrm{d} \\
\text { mixed nuts§ }\end{array}$ & $\begin{array}{l}\text { Glucose }(75 \mathrm{~g}) \mathrm{NS} \\
\text { Glucose }(37 \mathrm{~g}) \mathrm{NS} \\
\text { HbA1c }(75 \mathrm{~g}) \mathrm{NS} \\
\mathrm{HbA} 1 \mathrm{c}(37 \mathrm{~g}) \mathrm{NS}\end{array}$ & $\begin{array}{l}\text { NS-G; } 0.2 \\
\text { NS-G; } 0.2 \\
\text { NS-G; } 0.0 \\
\text { NS-G; } 0.0\end{array}$ \\
\hline $\begin{array}{l}\text { Thomazella et al. } \\
(2011)^{(48)}\end{array}$ & 12 weeks & $\begin{array}{c}n 40 \text { (control }=19, \text { Med } \\
\text { diet }=21), M, C V D \\
\text { mean } 55 \text { (SD 5) years }\end{array}$ & $\begin{array}{l}\text { Prospective controlled } \\
\text { study, Med diet (includ- } \\
\text { ing nuts) v. LF diet } \\
\text { (control) }\end{array}$ & $10 \mathrm{~g} / \mathrm{d}$ any nuts & Glucose NS & NS-G; 0.4 \\
\hline
\end{tabular}


Table 3. Continued

\begin{tabular}{|c|c|c|c|c|c|c|}
\hline Author & Time & Participants & Study design & Amount/type of nuts & Outcomes $\uparrow$ & $\begin{array}{l}\text { Effect; } \\
\text { effect size }\end{array}$ \\
\hline $\begin{array}{l}\text { Mercanligil et al. } \\
(2007)^{(74)}\end{array}$ & $\begin{array}{l}4 \text { weeks } \\
\text { per arm }\end{array}$ & $\begin{array}{l}n 15, \mathrm{M}, \text { mean } 48 \text { (SD } 8) \\
\text { years, hypercholestero- } \\
\text { laemic }\end{array}$ & $\begin{array}{l}\text { Two-period study, LF diet } \\
\text { (control) v. LF + hazel- } \\
\text { nuts, non-isoenergetic }\end{array}$ & $\begin{array}{r}40 \mathrm{~g} / \mathrm{d} \text { hazelnuts } \\
(12 \% \text { energy })\end{array}$ & Glucose NS & NS-G; 0.3 \\
\hline $\begin{array}{l}\text { Llorente-Cortés } \\
\text { et al. }(2011)^{(67)}\end{array}$ & 12 weeks & $\begin{array}{l}n 49(\mathrm{OO}=16, \text { nut }=15 \\
\text { control }=15), \text { high risk } \\
\text { of } C V D, M \text { and } F, \text { mean } \\
66(S D 7) \text { years }\end{array}$ & $\begin{array}{l}\text { RCT, parallel, Med diet + } \\
\text { OO v. Med diet + nuts } \\
\text { v. LF diet (control) } \\
\text { (PREDIMED) }\end{array}$ & $30 \mathrm{~g} / \mathrm{d}$ mixed nuts $\ddagger$ & Glucose NS & NS-G; 0.4 \\
\hline $\begin{array}{l}\text { Wien et al. } \\
(2003)^{(76)}\end{array}$ & 24 weeks & $\begin{array}{l}n 52 \text { (control }=28, \text { nut } \\
\quad=24), M \text { and } F \text {, over- } \\
\text { weight/obese, mean } 55 \\
\text { (SD 2) years }\end{array}$ & $\begin{array}{l}\mathrm{RCT} \text {, parallel, iso- } \\
\text { energetic, LE diet }+ \\
\text { almond } v \text {. CHO (control) }\end{array}$ & $84 \mathrm{~g} / \mathrm{d}$ almonds & $\begin{array}{l}\text { Glucose NS } \\
\text { Insulin NS } \\
\text { HOMA NS } \\
\text { HbA1c NS }\end{array}$ & $\begin{array}{l}\text { NS-G; } 0 \cdot 0 \\
\text { NS-G; } 0 \cdot 3 \\
\text { NS-G; } 0.0 \\
\text { NS-G; } 0 \cdot 0\end{array}$ \\
\hline $\begin{array}{l}\text { Zaveri \& Drum- } \\
\text { mond }(2009)^{(63)}\end{array}$ & 12 weeks & $\begin{array}{l}n 36 \text { (control = } 13, \text { cereal } \\
\text { bar }=14, \text { almond } \\
=18), \text { healthy, } M \text { and } F \\
\text { mean } 40(\mathrm{SD} 7) \text { years }\end{array}$ & $\begin{array}{l}\mathrm{RCT} \text {, parallel, healthy diet } \\
\text { with/without nuts or } \\
\text { cereal bar (control) }\end{array}$ & $56 \mathrm{~g} / \mathrm{d}$ almonds & $\begin{array}{l}\text { Glucose NS } \\
\text { Insulin NS }\end{array}$ & $\begin{array}{l}\text { NS-G } \\
\text { NS-G }\end{array}$ \\
\hline Li et al. $(2010)^{(75)}$ & 12 weeks & $\begin{array}{l}n 31, \text { obese, } \mathrm{M} \text { and } \mathrm{F}, \\
\quad \text { (control = } 28 \text { pistachio } \\
=31 \text { ), mean } 45 \text { (SD } 7) \\
\text { years }\end{array}$ & $\begin{array}{l}\mathrm{RCT} \text {, parallel, isoenergetic } \\
\text { prescribed diet }+ \\
\text { pretzels (control) or } \\
\text { pistachios }\end{array}$ & $53 \mathrm{~g} / \mathrm{d}$ pistachios & $\begin{array}{l}\text { Glucose NS } \\
\text { Insulin NS }\end{array}$ & $\begin{array}{l}\text { NS-G; }-0.6 \\
\text { NS-G; } 1.8\end{array}$ \\
\hline $\begin{array}{l}\text { Tapsell et al. } \\
(2004)^{(69)}\end{array}$ & 6 months & $\begin{array}{l}n 41 \text { (control }=21, \\
\text { walnut }=20), \mathrm{Dm}, \mathrm{M} \\
\text { and } \mathrm{F}, \text { mean } 60(\mathrm{SD} 8) \\
\text { years }\end{array}$ & $\begin{array}{l}\mathrm{RCT} \text {, parallel, isoenergetic } \\
\text { LF diet (control) } \\
\text { with/without walnuts }\end{array}$ & $30 \mathrm{~g} / \mathrm{d}$ walnuts & $\mathrm{HbA1c} N S$ & NS-G; 0.0 \\
\hline $\begin{array}{l}\text { Tapsell et al. } \\
(2009)^{(70)}\end{array}$ & 12 months & $\begin{array}{l}n 34 \text { (control }=17, \\
\text { walnut }=17) \text {, over- } \\
\text { weight, } \mathrm{Dm}, \mathrm{M} \text { and } \mathrm{F}, \\
\text { mean } 55(\mathrm{SD} 9) \text { years }\end{array}$ & $\begin{array}{l}\mathrm{RCT} \text {, parallel, isoenergetic } \\
\text { LF diet (control) } \\
\text { with/without walnuts }\end{array}$ & $30 \mathrm{~g} / \mathrm{d}$ walnuts & $\begin{array}{l}\text { Glucose NS } \\
\text { Insulin } \uparrow 6 \% * \\
\text { HbA1c NS }\end{array}$ & $\begin{array}{l}\text { NS-G; } 0.4 \\
\text { NS-G; }-0.3 \\
\text { NS-G; } 0.1\end{array}$ \\
\hline $\begin{array}{l}\text { Mukuddem-Peter- } \\
\text { sen et al. }(2007)^{(71)}\end{array}$ & 8 weeks & $\begin{array}{l}n 64 \text { (control }=22, \\
\text { walnut }=21, \text { cashew } \\
=21), \text { Met-S, } M \text { and F, } \\
\text { mean } 45 \text { (SD } 8 \text { ) years }\end{array}$ & $\begin{array}{l}\text { RCT, parallel, isoenergetic } \\
\text { LF diet (control) } \\
\text { with/without walnuts or } \\
\text { cashews }\end{array}$ & $\begin{array}{l}63-108 \mathrm{~g} / \mathrm{d} \text { walnuts/ } \\
\text { cashews }\end{array}$ & $\begin{array}{l}\text { Cashew } \\
\text { glucose } \uparrow 13 \% * \\
\text { Walnut glucose NS }\end{array}$ & $\begin{array}{l}\text { NS-G; }-0.8 \\
\text { NS-G; }-0.6\end{array}$ \\
\hline Ma et al. $(2010)^{(72)}$ & $\begin{array}{l}8 \text { weeks } \\
\text { per arm }\end{array}$ & $\begin{array}{l}n 21, \mathrm{Dm}, \mathrm{M} \text { and } \mathrm{F}, \text { mean } \\
58(\mathrm{SD} 8) \text { years }\end{array}$ & $\begin{array}{l}\text { RCT, cross-over, } \\
\text { ad libitum diet (control) } \\
\text { with/without nuts, not } \\
\text { isoenergetic, } 8 \text { weeks } \\
\text { washout }\end{array}$ & $56 \mathrm{~g} / \mathrm{d}$ walnuts & $\begin{array}{l}\text { Glucose NS } \\
\text { Insulin Ns } \\
\text { HOMA NS } \\
\text { HbA1c NS }\end{array}$ & $\begin{array}{l}\text { NS-G; }-0.3 \\
\text { NS-G; } 0.7 \\
\text { NS-G; }-0.4 \\
\text { NS-G; } 0.0\end{array}$ \\
\hline $\begin{array}{l}\text { Kasim-Karakas } \\
\text { et al. }(2004)^{(73)}\end{array}$ & 3 months & $\begin{array}{l}n 17, \text { mean } 34 \text { (SD 5) } \\
\text { years, F, PCOS }\end{array}$ & $\begin{array}{l}\text { Prospective cohort, iso- } \\
\text { energetic habitual diet } \\
\text { (control) } v \text {. walnuts }\end{array}$ & $106 \mathrm{~g} / \mathrm{d}$ walnuts & $\begin{array}{l}\text { Glucose } \uparrow 19 \%{ }^{*} \\
\text { Insulin NS } \\
\text { HOMA NS }\end{array}$ & $\begin{array}{l}-;-4.5 \\
\text { NS-G; } 0.2 \\
\text { NS-G; } 1.0\end{array}$ \\
\hline
\end{tabular}

Glucose, fasting glucose; M, male; F, female; Dm, type 2 diabetes; Med diet, Mediterranean diet; $\downarrow$, reduction; RR, relative risk; +, significant reduction; X-sect, cross-sectional; PREDIMED, PREvencion con Dleta MEDiterranea; N/A, not available; NS-G, no significant change; MESA, Multi-Ethnic Study of Atherosclerosis; NHANES, National Health and Nutrition Survey; RCT, randomised controlled trial; OO, olive oil; LF, low-fat; Met-S, metabolic syndrome; insulin, fasting insulin; HOMA, homeostatic model assessment of insulin resistance; NS, no significant difference; PCOS, polycystic ovary syndrome; HF, high-fat; LE, low-energy; CHO, carbohydrate; $\uparrow$, increase; -, significant increase

${ }^{*} P \leq 0.05$.

† Outcome (active $v$. control) for intervention studies.

F Mixed nuts = walnuts, almonds and hazelnuts.

$\S$ Mixed nuts $=$ almonds, pistachios, walnuts, groundnuts, hazelnuts, pecans, cashews and macadamias. 
Table 4. Studies measuring effect of nut consumption on blood pressure (BP)

\begin{tabular}{|c|c|c|c|c|c|c|c|}
\hline Author & Time & Subjects & Study design & Amount/type of nuts & Outcomes† & & $\begin{array}{l}\text { Effect; } \\
\text { effect size }\end{array}$ \\
\hline \multicolumn{8}{|c|}{ Prospective cohort studies measuring the effect of nut consumption on $\mathrm{HT}$ (studies are presented in order of efficacy) } \\
\hline \multirow[t]{2}{*}{$\begin{array}{l}\text { Steffen et al. } \\
(2005)^{(78)}\end{array}$} & \multirow[t]{2}{*}{15 years } & \multirow[t]{2}{*}{$\begin{array}{l}n 4304, \mathrm{M} \text { and } \mathrm{F} \text {, } \\
\text { healthy, } 18-30 \text { years }\end{array}$} & \multirow[t]{2}{*}{$\begin{array}{l}\text { Prospective cohort } \\
\text { (CARDIA), diet history } \\
\text { and prevalence of HT }\end{array}$} & $\begin{array}{l}\text { Any nuts } \\
<0.1 \text { serves } / d\end{array}$ & $\begin{array}{l}\mathrm{HR} \\
1.0\end{array}$ & \multirow{2}{*}{$\begin{array}{l}\text { Inverse relationship } \\
\text { between nut consump- } \\
\text { tion and HT } \\
P \text { for trend }=0.04\end{array}$} & \multirow[t]{2}{*}{+} \\
\hline & & & & $\begin{array}{l}0.1-0.3 \text { serves } / d \\
>0.3 \text { serves } / d\end{array}$ & $\begin{array}{l}0.84(95 \% \mathrm{Cl} 0.73,0.98) \\
0.85(95 \% \mathrm{Cl} 0.72,0.99)\end{array}$ & & \\
\hline $\begin{array}{l}\text { Djoussé et al. } \\
(2010)^{(77)}\end{array}$ & 12 months & $\begin{array}{l}n 15966, \text { free of } \mathrm{HT}, \mathrm{M} \\
\text { and } \mathrm{F}, \text { mean } 52 \\
\text { (range } 45-64 \text { ) years }\end{array}$ & $\begin{array}{l}\text { Prospective cohort, FFQ } \\
\text { and self-reported risk } \\
\text { of HT (Physicians' } \\
\text { Health Study) }\end{array}$ & $\begin{array}{l}\text { Any nuts } \\
\text { None } \\
1-3 \text { serves/month } \\
1 \text { serves/week } \\
2-6 \text { serves/week } \\
\geq 7 \text { serves/week }\end{array}$ & $\begin{array}{l}\text { HR } \\
1.0 \\
0.93(95 \% \mathrm{Cl} 0.86,1.01) \\
0.94(95 \% \mathrm{Cl} 0.86,1.03) \\
0.87(95 \% \mathrm{Cl} 0.79,0.96) \\
0.77(95 \% \mathrm{Cl} 0.64,0.93)\end{array}$ & $\begin{array}{l}\text { Nut consumption associ- } \\
\text { ated with } \downarrow \text { risk of } \\
\text { hypertension }(P=0.01)\end{array}$ & + \\
\hline $\begin{array}{l}\text { Weng et al. } \\
(2013)^{(79)}\end{array}$ & 9 years & $\begin{array}{l}n 9913 \text {, healthy, } M \text { and } \\
F, \text { mean } 53 \text { (SD 6) } \\
\text { years }\end{array}$ & $\begin{array}{l}\text { Prospective cohort, } \\
\text { ARIC, FFQ and BP }\end{array}$ & $\begin{array}{l}\text { Quintiles of nut } \\
\text { consumption }\end{array}$ & $\begin{array}{l}\text { HR } \\
1.2(95 \% \mathrm{Cl} 0.98,1.27) \\
0.96(95 \% \mathrm{Cl} 0.87,1.07) \\
0.91(95 \% \mathrm{Cl} 0.81,1.03) \\
0.87(95 \% \mathrm{Cl} 0.77,0.97)\end{array}$ & $\begin{array}{l}\text { Nut intake inversely } \\
\text { related to incidence of } \\
\text { HT }(P=0.02)\end{array}$ & + \\
\hline $\begin{array}{l}\text { Martínez-Lapiscina } \\
\text { et al. }(2010)^{(80)}\end{array}$ & 4 years & $\begin{array}{l}n 17177, \text { healthy, } M \text { and } \\
F, \text { mean } 36 \text { (range } \\
25-51 \text { ) years }\end{array}$ & $\begin{array}{l}\text { Prospective cohort } \\
\text { (SUN) study, FFQ and } \\
\text { self-reported HT }\end{array}$ & $\begin{array}{l}\text { Any nuts, never/rarely to } \\
2+/ \text { week }\end{array}$ & $\begin{array}{l}\text { HR highest } v \text {. lowest } \\
\text { intake }\end{array}$ & $\begin{array}{l}\text { No association with } \mathrm{HT} \\
\text { and nut consumption }\end{array}$ & NS-BP \\
\hline \multicolumn{8}{|c|}{ Cross-sectional studies measuring effect of nut consumption on BP/HT (studies are presented in order of efficacy) } \\
\hline \multirow[t]{4}{*}{$\begin{array}{l}\text { O'Neil et al. } \\
(2011)^{(49)}\end{array}$} & \multirow[t]{4}{*}{$X$-sect } & $\begin{array}{l}n 13292, \text { general } \\
\text { population, } M \text { and } F \text {, } \\
19-50+\text { years }\end{array}$ & \multirow[t]{4}{*}{$\begin{array}{l}1999-2004 \text { NHANES, } \\
24 \mathrm{~h} \text { recall and BP }\end{array}$} & & HT v. non-consumers & $\begin{array}{l}\text { BP } v \text {. non-consumers } \\
\text { (A) SBP } \downarrow 1 \mathrm{mmHg} \\
(P<0.01)\end{array}$ & + \\
\hline & & \multirow[t]{3}{*}{ (20\% nut consumers) } & & $\begin{array}{l}\text { 'All' nut group } \geq 7 \mathrm{~g} / \mathrm{d} \\
\text { (A) }\end{array}$ & $\downarrow 3 \% *$ & (A) DBP NS & NS-BP \\
\hline & & & & \multirow[t]{2}{*}{$\begin{array}{l}\text { Tree nut group } \geq 7 \mathrm{~g} / \mathrm{d} \\
\text { (B) }\end{array}$} & \multirow[t]{2}{*}{$\downarrow 3 \% *$} & $\begin{array}{l}\text { (B) SBP } \downarrow 1 \mathrm{mmHg} \\
(P<0.01)\end{array}$ & + \\
\hline & & & & & & (B) DBP NS & NS-BP \\
\hline $\begin{array}{l}\text { Nettleton et al. } \\
(2008)^{(52)}\end{array}$ & X-sect & $\begin{array}{l}n 5089 \text {, healthy, } M \text { and } \\
F, 45-84 \text { years }\end{array}$ & MESA, FFQ and BP & $\begin{array}{l}\text { Quintiles of healthy diet- } \\
\text { ary pattern (including } \\
\text { any nuts) }\end{array}$ & No data provided & $\begin{array}{l}\text { No association of SBP or } \\
\text { DBP with healthy diet- } \\
\text { ary pattern }\end{array}$ & NS-BP \\
\hline $\begin{array}{l}\text { Alvarez León et al. } \\
(2006)^{(50)}\end{array}$ & $X$-sect & $\begin{array}{l}n 578, \text { healthy or Met-S, } \\
M \text { and } F, 18-75 \text { years }\end{array}$ & ENCA, FFQ and BP & $\begin{array}{l}\text { Tertiles (any nuts) } \\
\text { Nuts T1 } \\
\text { Nuts T2 } \\
\text { Nuts T3 }\end{array}$ & $\begin{array}{l}\text { OR HT } \\
1.0 \\
0.73(95 \% \mathrm{Cl} 0.41,1.28) \\
0.83(95 \% \mathrm{Cl} 0.47,1.46)\end{array}$ & $\begin{array}{l}\text { No association of BP } \\
\text { with nut consumption }\end{array}$ & NS-BP \\
\hline $\begin{array}{l}\text { Ibarrola-Jurado } \\
\text { et al. }(2013)^{(81)}\end{array}$ & $X$-sect & $\begin{array}{l}n 7210, \text { high risk of } C V D \text {, } \\
M \text { and } F \text {, mean } 68 \\
\text { (SD 6) years }\end{array}$ & $\begin{array}{l}\text { X-sect, FFQ and } \\
\text { prevalence of HT } \\
\text { (PREDIMED) }\end{array}$ & $\begin{array}{l}\text { Tertiles (any nuts) } \\
\text { T1 }<28 \mathrm{~g} / \text { week } \\
\text { T2 } 28-84 \mathrm{~g} / \mathrm{week} \\
\text { T3 }>84 \mathrm{~g} / \text { week }\end{array}$ & $\begin{array}{l}\text { OR HT } \\
1.0 \\
0.96(95 \% \mathrm{Cl} 0.71,1.29) \\
1.12(95 \% \mathrm{Cl} 0.81,1.53)\end{array}$ & $\begin{array}{l}\text { No association of } \mathrm{HT} \\
\text { with nut consumption }\end{array}$ & NS-BP \\
\hline \multicolumn{8}{|c|}{ Chronic effects of nuts on resting BP (studies are presented in order of efficacy) } \\
\hline $\begin{array}{l}\text { Wien et al. } \\
(2003)^{(76)}\end{array}$ & 24 weeks & $\begin{array}{l}n 52 \text { (control }=28 \text { nut } \\
=24), M \text { and } F, \text { over- } \\
\text { weight/obese, mean } \\
55(\mathrm{SD} 2) \text { years }\end{array}$ & $\begin{array}{l}\text { RCT, parallel, iso- } \\
\text { energetic, LE diet }+ \\
\text { almond } v \text {. CHO } \\
\text { (control) }\end{array}$ & $84 \mathrm{~g} / \mathrm{d}$ almonds & $\begin{array}{l}\text { SBP } \downarrow 14 \mathrm{mmHg}^{*} \\
\text { DBP NS }\end{array}$ & & $\begin{array}{l}+; 0.7 \\
\text { NS-BP; } 0.0\end{array}$ \\
\hline $\begin{array}{l}\text { Estruch et al. } \\
(2006)^{(58)}\end{array}$ & 12 weeks & $\begin{array}{l}n 772 \text { (control }=257 \\
\text { OO = } 257, \text { nut } \\
=258 \text { ), high risk of } \\
\text { CVD, M and F, mean } \\
69 \text { (SD 6) years }\end{array}$ & $\begin{array}{l}\text { RCT, parallel, Med diet } \\
\text { + OO } v \text {. Med diet }+ \\
\text { nuts } v \text {. LF diet } \\
\text { (control) (PREDIMED) }\end{array}$ & $30 \mathrm{~g} / \mathrm{d}$ mixed nuts $\ddagger$ & $\begin{array}{l}\text { SBP } \downarrow 7 \mathrm{mmHg}^{*} \\
\mathrm{DBP} \downarrow 3 \mathrm{mmHg}^{\star}\end{array}$ & & $\begin{array}{l}+; 0.3 \\
+; 0.2\end{array}$ \\
\hline
\end{tabular}




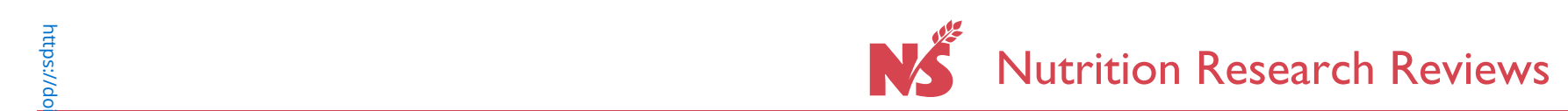

Table 4. Continued

\begin{tabular}{|c|c|c|c|c|c|c|}
\hline Author & Time & Subjects & Study design & Amount/type of nuts & Outcomes $\dagger$ & $\begin{array}{l}\text { Effect; } \\
\text { effect size }\end{array}$ \\
\hline $\begin{array}{l}\text { Esposito et al. } \\
(2004)^{(47)}\end{array}$ & 2 years & $\begin{array}{l}n 180 \text { (control }=90, \\
\text { Med diet }=90), \\
\text { Met-S, M and F, } \\
\text { mean } 44 \text { (SD 6) years }\end{array}$ & $\begin{array}{l}\text { RCT, parallel, Med diet } \\
\text { (including nuts) } \\
\text { v. prudent diet } \\
\text { (control) }\end{array}$ & $20-50 \mathrm{~g} / \mathrm{d}$ walnuts & $\begin{array}{l}\text { SBP } \downarrow 3 \mathrm{mmHg}^{\star} \\
\text { DBP } \downarrow 2 \mathrm{mmHg}^{*}\end{array}$ & $\begin{array}{l}+; 0.7 \\
+; 0.7\end{array}$ \\
\hline $\begin{array}{l}\text { Adamsson et al. } \\
(2011)^{(46)}\end{array}$ & 4 weeks & $\begin{array}{l}n 86(\text { control }=42, \\
\text { NORDIET }=44), \mathrm{M} \\
\text { and F, hypercholes- } \\
\text { terolaemic, mean } 53 \\
\text { (SD 8) years }\end{array}$ & $\begin{array}{l}\text { RCT, parallel, NORDIET } \\
\text { (high fibre, fish, LF } \\
\text { dairy, nuts) v. habitual } \\
\text { diet (control) }\end{array}$ & Ad libitum almonds & $\begin{array}{l}\text { SBP } \downarrow 6 \mathrm{mmHg}^{\star} \\
\text { DBP } \downarrow 3 \mathrm{mmHg}^{\star}\end{array}$ & $\begin{array}{l}+; 0.6 \\
+; 0.4\end{array}$ \\
\hline $\begin{array}{l}\text { Llorente-Cortés } \\
\text { et al. }(2011)^{(67)}\end{array}$ & 12 weeks & $\begin{array}{l}n 49(\mathrm{OO}=16, \text { nut } \\
=15, \text { control }=15), \\
\text { high risk of CVD, M } \\
\text { and } \mathrm{F}, \text { mean } 66(\mathrm{SD} 7) \\
\text { years }\end{array}$ & $\begin{array}{l}\text { RCT, parallel, Med diet } \\
+ \text { OO v. Med diet + } \\
\text { nuts } v . \text { LF diet } \\
\text { (control) (PREDIMED) }\end{array}$ & $30 \mathrm{~g} / \mathrm{d}$ mixed nuts $\ddagger$ & $\begin{array}{l}\text { SBP } \downarrow 7 \mathrm{mmHg}^{*} \\
\text { DBP NS }\end{array}$ & $\begin{array}{l}+; 0.4 \\
\text { NS-BP; } 0.2\end{array}$ \\
\hline $\begin{array}{l}\text { Fito et al. } \\
(2007)^{(85)}\end{array}$ & 12 weeks & $\begin{array}{l}n 372(\mathrm{OO}=123, \text { nut } \\
=128, \text { control }=127), \\
\text { high risk of CVD, M } \\
\text { and } F, \text { mean } 66(\text { SD } 9) \\
\text { years }\end{array}$ & $\begin{array}{l}\text { RCT, parallel, Med diet } \\
+ \text { OO v. Med diet + } \\
\text { nuts } v . \text { LF diet } \\
\text { (control) (PREDIMED) }\end{array}$ & $30 \mathrm{~g} / \mathrm{d}$ mixed nuts $\ddagger$ & $\begin{array}{l}\text { SBP } \downarrow^{*}(\text { data N/A }) \\
\text { DBP } \downarrow^{*}(\text { data N/A })\end{array}$ & $\begin{array}{l}+ \\
+\end{array}$ \\
\hline $\begin{array}{l}\text { Jenkins et al. } \\
(2008)^{(82)}\end{array}$ & 1 year & $\begin{array}{l}n 50, \text { hyperlipidaemic, } M \\
\text { and } \mathrm{F} \text {, mean } 59 \text { (SD 1) } \\
\text { years }\end{array}$ & $\begin{array}{l}\text { Single-phase prospective } \\
\text { study, pre-/post- } \\
\text { measures, sterol + } \\
\text { soya }+ \text { almonds } \\
\text { (no control) }\end{array}$ & $\begin{array}{l}23 \mathrm{~g} / 4.2 \mathrm{MJ} \text { per } \mathrm{d} \\
\quad \text { almonds }\end{array}$ & $\begin{array}{l}\text { (v.. baseline) } \\
\text { SBP } \downarrow 4 \mathrm{mmHg}^{*} \\
\text { DBP } \downarrow 2 \mathrm{mmHg}^{*}\end{array}$ & $\begin{array}{l}+; 0.4 \\
+; 0.5\end{array}$ \\
\hline $\begin{array}{l}\text { Mena et al. } \\
(2009)^{(84)}\end{array}$ & 12 weeks & $\begin{array}{l}n 106(\text { control }=36, \text { nut } \\
=35, \mathrm{OO}=35), \mathrm{Dm}, \\
\text { risk of } \mathrm{CVD}, \mathrm{M} \text { and } \mathrm{F}, \\
\text { mean } 66(\mathrm{SD} 7) \text { years }\end{array}$ & $\begin{array}{l}\text { RCT, parallel, Med diet } \\
\text { + OO v. Med diet + } \\
\text { nuts v. LF diet } \\
\text { (control) (PREDIMED) }\end{array}$ & $30 \mathrm{~g} / \mathrm{d}$ mixed nuts $\ddagger$ & $\begin{array}{l}\text { ( } v \text {. baseline) } \\
\text { SBP } \downarrow 3 \mathrm{mmHg}^{*} \\
\text { DBP } \downarrow 2 \mathrm{mmHg}^{*}\end{array}$ & $\begin{array}{l}+ \\
+\end{array}$ \\
\hline $\begin{array}{l}\text { Toledo et al. } \\
(2013)^{(83)}\end{array}$ & 4 years & $\begin{array}{l}n 7158 \text { (control }=2064, \\
O O=2345, \text { nuts } \\
=2065), M \text { and } F, \text { risk } \\
\text { of CVD, mean } 67 \\
\text { (SD 6) years }\end{array}$ & $\begin{array}{l}\text { RCT, parallel, Med diet } \\
+ \text { OO v. Med diet + } \\
\text { nuts } v . \text { LF diet } \\
\text { (control) (PREDIMED) }\end{array}$ & $30 \mathrm{~g} / \mathrm{d}$ mixed nuts $\ddagger$ & $\begin{array}{l}\text { SBP NS } \\
\text { DBP } \downarrow 2 \mathrm{mmHg}^{*}\end{array}$ & $\begin{array}{l}\text { NS-BP; } 0.2 \\
+; 1 \cdot 1\end{array}$ \\
\hline $\begin{array}{l}\text { Wien et al. } \\
(2010)^{(61)}\end{array}$ & 16 weeks & $\begin{array}{l}n 65 \text { (control }=32, \text { nut } \\
=33 \text { ), prediabetes, } \\
M \text { and F, mean } 53 \\
\text { (SD 9) years }\end{array}$ & $\begin{array}{l}\text { RCT, parallel, American } \\
\text { Diabetes Association } \\
\text { diet (control) with/ } \\
\text { without almonds }\end{array}$ & $56 \mathrm{~g} / \mathrm{d}$ almonds & $\begin{array}{l}\text { SBP NS } \\
\text { DBP NS }\end{array}$ & $\begin{array}{l}\text { NS-BP; } 0.2 \\
\text { NS-BP; } 0.2\end{array}$ \\
\hline $\begin{array}{l}\text { Jenkins et al. } \\
(2011)^{(66)}\end{array}$ & 3 months & $\begin{array}{l}n 117 \text { (control }=37, \\
37 \mathrm{~g} / \mathrm{d} \text { nut }=40, \\
75 \mathrm{~g} / \mathrm{d} \text { nuts }=40), \\
\text { Dm, } \mathrm{M} \text { and } \mathrm{F}, \text { mean } \\
62(\mathrm{sD} 10) \text { years }\end{array}$ & $\begin{array}{l}\text { RCT, parallel, } 75 \mathrm{~g} / \mathrm{d} \text { nut } \\
\text { v. } 37 \mathrm{~g} / \mathrm{d} \text { nuts }+ \\
\text { half-dose muffin } \\
\text { v. muffin (control) }\end{array}$ & 75 or $37 \mathrm{~g} / \mathrm{d}$ mixed nuts§ & $\begin{array}{l}\text { SBP NS } \\
\text { DBP NS }\end{array}$ & $\begin{array}{l}\text { NS-BP; } 0.2 \\
\text { NS-BP; } 0.2\end{array}$ \\
\hline $\begin{array}{l}\text { Spaccarotella et al. } \\
(2008)^{(146)}\end{array}$ & $\begin{array}{l}8 \text { weeks per } \\
\text { arm }\end{array}$ & $\begin{array}{l}n 21, \text { healthy, } M \text { and } F \text {, } \\
\text { mean } 66 \text { (range } \\
45-75 \text { ) years }\end{array}$ & $\begin{array}{l}\text { RCT, cross-over, habit- } \\
\text { ual diet (control) with/ } \\
\text { without walnuts } \\
\text { ( } 2 \text { weeks washout) }\end{array}$ & $75 \mathrm{~g} / \mathrm{d}$ walnuts & $\begin{array}{l}\text { SBP NS } \\
\text { DBP NS }\end{array}$ & $\begin{array}{l}\text { NS-BP; } 0.1 \\
\text { NS-BP; } 0.2\end{array}$ \\
\hline Wu et al. $(2010)^{(68)}$ & 12 weeks & $\begin{array}{l}n 277 \text { (control }=95 \\
\text { flax }=94, \text { nut }=94) \\
\text { Met-S, M and F, mean } \\
49(\text { SD 8) years }\end{array}$ & $\begin{array}{l}\text { RCT, parallel, } \\
\text { isoenergetic LF diet } \\
\text { (control) with/without } \\
\text { flaxseeds or walnuts }\end{array}$ & $30 \mathrm{~g} / \mathrm{d}$ walnut flour & $\begin{array}{l}\text { SBP NS } \\
\text { DBP NS }\end{array}$ & $\begin{array}{l}\text { NS-BP; } 0 \cdot 1 \\
\text { NS-BP; } 0 \cdot 0\end{array}$ \\
\hline
\end{tabular}




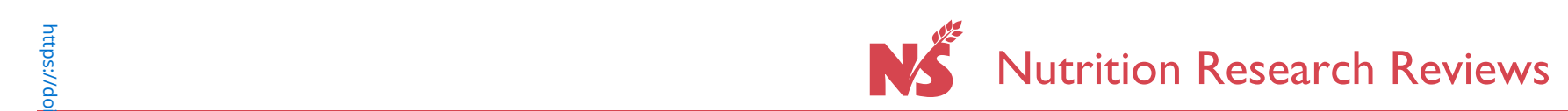

Table 4. Continued

\begin{tabular}{|c|c|c|c|c|c|c|}
\hline Author & Time & Subjects & Study design & Amount/type of nuts & Outcomes† & $\begin{array}{l}\text { Effect; } \\
\text { effect size }\end{array}$ \\
\hline $\begin{array}{l}\text { Sari et al. } \\
(2010)^{(59)}\end{array}$ & $\begin{array}{l}4 \text { weeks per } \\
\text { arm }\end{array}$ & $\begin{array}{l}n 32, \text { healthy, } M, \text { mean } \\
22 \text { (range } 21-24 \text { ) } \\
\text { years }\end{array}$ & $\begin{array}{l}\text { Prospective cohort, } \\
\text { isoenergetic Med diet } \\
\text { (control) with/without } \\
\text { pistachios, no washout }\end{array}$ & $80-100 \mathrm{~g} / \mathrm{d}$ pistachios & $\begin{array}{l}\text { SBP NS } \\
\text { DBP NS }\end{array}$ & $\begin{array}{l}\text { NS-BP; } 0.3 \\
\text { NS-BP; } 0.1\end{array}$ \\
\hline $\begin{array}{l}\text { Din et al. } \\
(2011)^{(109)}\end{array}$ & $\begin{array}{l}4 \text { weeks per } \\
\text { arm }\end{array}$ & $\begin{array}{l}n 30, \text { healthy, } M \text { and } F \text {, } \\
\text { mean } 23 \text { (SD 3) years }\end{array}$ & $\begin{array}{l}\text { RCT, cross-over, } \\
\text { non-isoenergetic } \\
\text { habitual diet (control) } \\
\text { with/without walnuts, } \\
\text { no washout }\end{array}$ & $15 \mathrm{~g} / \mathrm{d}$ walnuts & $\begin{array}{l}\text { SBP NS } \\
\text { DBP NS }\end{array}$ & $\begin{array}{l}\text { NS-BP; } 0.3 \\
\text { NS-BP; }-0.1\end{array}$ \\
\hline $\begin{array}{l}\text { Thomazella et al. } \\
(2011)^{(48)}\end{array}$ & 12 weeks & $\begin{array}{c}n 40 \text { (control }=19, \text { Med } \\
\text { diet }=21), M, \text { CVD, } \\
\text { mean } 55(\text { SD 5) years }\end{array}$ & $\begin{array}{l}\text { Prospective controlled } \\
\text { study, Med diet } \\
\text { (including nuts) } \\
\text { v. LF diet (control) }\end{array}$ & $10 \mathrm{~g} / \mathrm{d}$ any nuts & $\begin{array}{l}\text { SBP NS } \\
\text { DBP NS }\end{array}$ & $\begin{array}{l}\text { NS-BP; } 0.2 \\
\text { NS-BP; } 0.1\end{array}$ \\
\hline $\begin{array}{l}\text { Mukuddem-Peter- } \\
\text { sen et al. }(2007)^{(71)}\end{array}$ & 8 weeks & $\begin{array}{l}n 64 \text { (control }=22, \text { nut } \\
=21, \text { cashew }=21) \\
\text { Met-S, } M \text { and } F, \text { mean } \\
45 \text { (SD 8) years }\end{array}$ & $\begin{array}{l}\text { RCT, parallel, iso- } \\
\text { energetic LF diet } \\
\text { (control) with/without } \\
\text { walnuts (W) or } \\
\text { cashews (C) }\end{array}$ & $\begin{array}{l}63-108 \mathrm{~g} / \mathrm{d} \text { walnuts/ca- } \\
\text { shews }\end{array}$ & $\begin{array}{l}\text { SBP NS W and C } \\
\text { DBP NS W and C }\end{array}$ & $\begin{array}{l}\text { NS-BP; } 0.1 \\
\text { NS-BP; } 0.1\end{array}$ \\
\hline $\begin{array}{l}\text { Iwamoto et al. } \\
(2002)^{(147)}\end{array}$ & $\begin{array}{l}4 \text { weeks per } \\
\text { arm }\end{array}$ & $\begin{array}{l}n 80, \text { healthy, } M \text { and } F, \\
\text { mean } 24 \text { (SD 9) years }\end{array}$ & $\begin{array}{l}\text { RCT, cross-over, } \\
\text { Japanese diet with/ } \\
\text { without walnuts } \\
\text { (no washout) }\end{array}$ & $44-58 \mathrm{~g} / \mathrm{d}$ walnuts & $\begin{array}{l}\text { SBP NS } \\
\text { DBP NS }\end{array}$ & $\begin{array}{l}\text { NS-BP } \\
\text { NS-BP }\end{array}$ \\
\hline $\begin{array}{l}\text { West et al. } \\
(2012)^{(107)}\end{array}$ & $\begin{array}{l}4 \text { weeks per } \\
\text { arm }\end{array}$ & $\begin{array}{l}n 25, \text { hypercholestero- } \\
\text { laemic, M and F, } \\
\text { mean } 48 \text { (SD 2) years }\end{array}$ & $\begin{array}{l}\text { RCT, cross-over, LF diet } \\
\text { (control) v. low-dose } \\
\text { pistachios v. high-dose } \\
\text { pistachios, no washout }\end{array}$ & $\begin{array}{l}32-63 \mathrm{~g} / \mathrm{d}(\mathrm{A}) \text { or } 63- \\
126 \mathrm{~g} / \mathrm{d}(\mathrm{B}) \text { pistachios }\end{array}$ & $\begin{array}{l}\text { (A) SBP NS } \\
\text { (A) DBP NS } \\
\text { (B) SBP NS } \\
\text { (B) DBP NS }\end{array}$ & $\begin{array}{l}\text { NS-BP; } 0.1 \\
\text { NS-BP; } 0.1 \\
\text { NS-BP; } 0.0 \\
\text { NS-BP; } 0 \cdot 0\end{array}$ \\
\hline $\begin{array}{l}\text { Jenkins et al. } \\
(2003)^{(89)}\end{array}$ & 4 weeks & $\begin{array}{l}n 25 \text { (control }=12, \text { nut } \\
=13 \text { ), hyperlipidae- } \\
\text { mic, M and F, mean } \\
59 \text { (SD 1) years }\end{array}$ & $\begin{array}{l}\text { RCT, parallel, plant } \\
\text { sterol }+ \text { soya }+ \\
\text { almond } v \text {. statin } \\
v . \text { LF diet (control) }\end{array}$ & $14 \mathrm{~g} / 4 \cdot 2 \mathrm{MJ}$ almonds & $\begin{array}{l}\text { SBP NS } \\
\text { DBP NS }\end{array}$ & $\begin{array}{l}\text { NS-BP; } 0.1 \\
\text { NS-BP; } 0.1\end{array}$ \\
\hline $\begin{array}{l}\text { Jenkins et al. } \\
(2002)^{(99)}\end{array}$ & $\begin{array}{l}1 \text { month per } \\
\text { arm }\end{array}$ & $\begin{array}{l}n 27, \text { hyperlipidaemic, M } \\
\text { and F, mean } 64 \text { (SD 9) } \\
\text { years }\end{array}$ & $\begin{array}{l}\text { RCT, cross-over, } \\
\text { isoenergetic almonds } \\
v \text {. half-dose almonds } \\
+ \text { half-dose muffin } \\
\text { v. muffin (control) }\end{array}$ & 36 or $73 \mathrm{~g} / \mathrm{d}$ almonds & $\begin{array}{l}\text { SBP NS } \\
\text { DBP NS }\end{array}$ & $\begin{array}{l}\text { NS-BP; } 0.0 \\
\text { NS-BP; } 0.0\end{array}$ \\
\hline $\begin{array}{l}\text { Hiraoka-Yamamoto } \\
\text { et al. }(2004)^{(148)}\end{array}$ & 3 weeks & $\begin{array}{l}n 71 \text { (control }=24, \\
\text { coconut }=23, \text { nut } \\
=24), F, \text { students, } \\
\text { mean } 19 \text { (SD 3) years }\end{array}$ & $\begin{array}{l}\text { RCT, parallel, coconut } \\
\text { v. macadamia } v \text {. butter } \\
\text { (control) }\end{array}$ & $10 \mathrm{~g} / \mathrm{d}$ macadamias & SBP NS & NS-BP; 0.0 \\
\hline $\begin{array}{l}\text { Damesceno et al. } \\
(2011)^{(149)}\end{array}$ & $\begin{array}{l}4 \text { weeks per } \\
\text { arm }\end{array}$ & $\begin{array}{l}n 18, \text { hypercholestero- } \\
\text { laemic, } M \text { and } F \text {, } \\
\text { mean } 56 \text { (SD 13) years }\end{array}$ & $\begin{array}{l}\text { RCT, cross-over, } \\
\text { isoenergetic Med diet } \\
+ \text { OO (control) } v \text {. Med } \\
\text { diet }+ \text { walnuts } v \text {. Med } \\
\text { diet + almonds, no } \\
\text { washout }\end{array}$ & $\begin{array}{l}40-65 \mathrm{~g} / \mathrm{d} \text { walnuts } \\
50-75 \mathrm{~g} / \mathrm{d} \text { almonds }\end{array}$ & $\begin{array}{l}\text { SBP NS } \\
\text { DBP NS } \\
\text { SBP NS } \\
\text { DBP NS }\end{array}$ & $\begin{array}{l}\text { NS-BP; } 0 \cdot 2 \\
\text { NS-BP; } 0 \cdot 2 \\
\text { NS-BP; } 0 \cdot 1 \\
\text { NS-BP; } 0 \cdot 2\end{array}$ \\
\hline $\begin{array}{l}\text { Olmedilla-Alonso } \\
\text { et al. }(2008)^{(150)}\end{array}$ & $\begin{array}{l}5 \text { weeks per } \\
\text { arm }\end{array}$ & $\begin{array}{l}n 25, \text { high risk of CVD, } \\
M \text { and } F \text {, mean } 54 \\
\text { (SD 8) years }\end{array}$ & $\begin{array}{l}\text { RCT, cross-over, meat } \\
\text { product with/without } \\
20 \% \text { walnut flour, } \\
\text { 1-month washout }\end{array}$ & $19 \mathrm{~g} / \mathrm{d}$ walnuts & $\begin{array}{l}\text { SBP NS } \\
\text { DBP NS }\end{array}$ & $\begin{array}{l}\text { NS-BP; }-0.2 \\
\text { NS-BP; } 0.0\end{array}$ \\
\hline
\end{tabular}




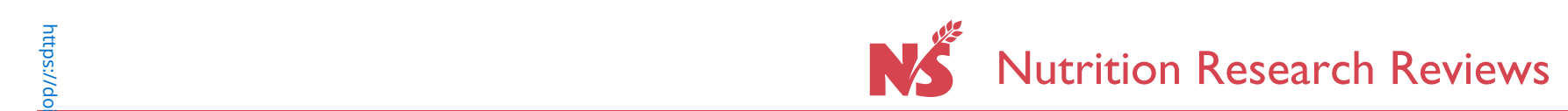

Table 4. Continued

\begin{tabular}{|c|c|c|c|c|c|c|}
\hline Author & Time & Subjects & Study design & Amount/type of nuts & Outcomes $\dagger$ & $\begin{array}{l}\text { Effect; } \\
\text { effect size }\end{array}$ \\
\hline $\begin{array}{l}\text { Casas-Agustench } \\
\text { et al. }(2011)^{(60)}\end{array}$ & 12 weeks & $\begin{array}{l}n 50 \text { (control }=25 \text { nut } \\
=25 \text { ), Met-S, M and } \\
F, \text { mean } 52 \text { (SD 8) } \\
\text { years }\end{array}$ & $\begin{array}{l}\text { RCT, parallel, } \\
\text { isoenergetic healthy } \\
\text { diet with/without nuts }\end{array}$ & $30 \mathrm{~g} / \mathrm{d}$ mixed nuts $\ddagger$ & $\begin{array}{l}\text { SBP NS } \\
\text { DBP NS }\end{array}$ & $\begin{array}{l}\text { NS-BP; }-0.2 \\
\text { NS-BP; }-0.1\end{array}$ \\
\hline $\begin{array}{l}\text { Nouran et al. } \\
(2010)^{(151)}\end{array}$ & 4 weeks & $\begin{array}{l}n 108 \text { (control }=54, \\
\text { groundnut }=54 \text { ), } \\
\text { hypercholesterolae- } \\
\text { mic, M and F, mean } \\
43 \text { (SD 10) years }\end{array}$ & $\begin{array}{l}\mathrm{RCT} \text {, parallel, habitual } \\
\text { diet (control) with/ } \\
\text { without groundnuts }\end{array}$ & $77 \mathrm{~g} / \mathrm{d}$ groundnuts & $\begin{array}{l}\text { SBP NS } \\
\text { DBP NS }\end{array}$ & $\begin{array}{l}\text { NS-BP; } 0.0 \\
\text { NS-BP; } 0.0\end{array}$ \\
\hline $\begin{array}{l}\text { Ros et al. } \\
(2004)^{(98)}\end{array}$ & $\begin{array}{l}4 \text { weeks per } \\
\text { arm }\end{array}$ & $\begin{array}{l}n 20, \text { hypercholestero- } \\
\text { laemic, M and F, } \\
\text { mean } 55 \text { (range } \\
26-75 \text { ) years }\end{array}$ & $\begin{array}{l}\text { RCT, cross-over, } \\
\text { isoenergetic Med diet } \\
\text { (control) with/without } \\
\text { walnuts, no washout }\end{array}$ & $40-65 \mathrm{~g} / \mathrm{d}$ walnuts & $\begin{array}{l}\text { SBP NS } \\
\text { DBP NS }\end{array}$ & $\begin{array}{l}\text { NS-BP; } 0 \cdot 0 \\
\text { NS-BP; } 0 \cdot 1\end{array}$ \\
\hline $\begin{array}{l}\text { López-Uriarte et al. } \\
(2010)^{(106)}\end{array}$ & 12 weeks & $\begin{array}{l}n 50 \text { (control }=25, \text { nut } \\
=25), \text { Met-S, M and } \\
F, \text { mean } 52 \text { (SD 8) } \\
\text { years }\end{array}$ & $\begin{array}{l}\text { RCT, parallel, American } \\
\text { Heart Association diet } \\
\text { (control) with/without } \\
\text { nuts, not isoenergetic }\end{array}$ & $30 \mathrm{~g} / \mathrm{d}$ mixed nuts $\ddagger$ & $\begin{array}{l}\text { SBP NS } \\
\text { DBP NS }\end{array}$ & $\begin{array}{l}\text { NS-BP } \\
\text { NS-BP }\end{array}$ \\
\hline $\begin{array}{l}\text { Solà et al. } \\
(2012)^{(86)}\end{array}$ & $\begin{array}{l}4 \text { weeks per } \\
\text { arm }\end{array}$ & $\begin{array}{l}n 113 \text { (control = } 28, \text { nut } \\
=28, \text { nut }+ \text { sterol } \\
=30, \text { nut }+ \text { sterol }+ \\
\text { fibre }=27 \text { ), risk of } \\
\text { CVD, M and F, mean } \\
54 \text { (SD 9) years }\end{array}$ & $\begin{array}{l}\text { RCT, parallel, iso- } \\
\text { energetic cocoa }+ \text { nut } \\
\text { v. cocoa }+ \text { nut }+ \\
\text { sterol } v . \text { cocoa }+ \text { nut } \\
+ \text { sterol + fibre } \\
\text { v. cocoa (control) }\end{array}$ & $30 \mathrm{~g} / \mathrm{d}$ hazelnuts & $\begin{array}{l}\text { SBP NS } \\
\text { DBP NS }\end{array}$ & $\begin{array}{l}\text { NS-BP } \\
\text { NS-BP }\end{array}$ \\
\hline $\begin{array}{l}\text { Sabate et al. } \\
(1993)^{(152)}\end{array}$ & $\begin{array}{l}4 \text { weeks per } \\
\text { arm }\end{array}$ & $\begin{array}{l}n 18, \text { healthy, } M, \text { mean } \\
30 \text { (range } 21-43 \text { ) } \\
\text { years }\end{array}$ & $\begin{array}{l}\text { RCT, cross-over, } \\
\text { isoenergetic LF diet } \\
\text { (control) with/without } \\
\text { walnuts, no washout }\end{array}$ & $\begin{array}{l}20 \% \text { energy walnuts } \\
(84 \mathrm{~g} / 4.4 \mathrm{MJ} \text { per } \mathrm{d})\end{array}$ & $\begin{array}{l}\text { SBP NS } \\
\text { DBP NS }\end{array}$ & $\begin{array}{l}\text { NS-BP } \\
\text { NS-BP }\end{array}$ \\
\hline $\begin{array}{l}\text { Edwards et al. } \\
(1999)^{(153)}\end{array}$ & $\begin{array}{l}3 \text { weeks per } \\
\text { arm }\end{array}$ & $\begin{array}{l}n 10, \text { hypercholestero- } \\
\text { laemic, } M \text { and } F, \\
\text { mean } 46 \text { (range } \\
41-64 \text { ) years }\end{array}$ & $\begin{array}{l}\text { RCT, cross-over, iso- } \\
\text { energetic habitual diet } \\
\text { (control) with/without } \\
\text { pistachios } \\
\text { (20\% energy) }\end{array}$ & (20\% energy) pistachios & $\begin{array}{l}\text { SBP NS } \\
\text { DBP NS }\end{array}$ & $\begin{array}{l}\text { NS-BP } \\
\text { NS-BP }\end{array}$ \\
\hline $\begin{array}{l}\text { Spiller et al. } \\
(2003)^{(154)}\end{array}$ & 4 weeks & $\begin{array}{c}n 38 \text { (raw }=14, \text { roasted } \\
=14 \text { butter }=10), M \\
\text { and } \mathrm{F}, 32-74 \text { years, } \\
\text { hypercholesterolaemic }\end{array}$ & $\begin{array}{l}\text { RCT, parallel, LF diet }+ \\
\text { nuts (roasted } \\
\text { v. roasted butter } \\
\text { v. raw (control)) }\end{array}$ & $\begin{array}{l}100 \mathrm{~g} / \mathrm{d} \text { almonds/almond } \\
\text { butter }\end{array}$ & $\begin{array}{l}\text { SBP NS } \\
\text { DBP NS }\end{array}$ & $\begin{array}{l}\text { NS-BP } \\
\text { NS-BP }\end{array}$ \\
\hline $\begin{array}{l}\text { Jenkins et al. } \\
(2002)^{(88)}\end{array}$ & $\begin{array}{l}4 \text { weeks per } \\
\text { arm }\end{array}$ & $\begin{array}{l}n 13 \text {, hyperlipidaemic, M } \\
\text { and } F \text {, mean } 65 \text { (SD 3) } \\
\text { years }\end{array}$ & $\begin{array}{l}\text { Prospective cohort, } \\
\text { LF diet followed by } \\
\text { portfolio diet (soya/ } \\
\text { plant sterol/fibre/nuts) }\end{array}$ & $\begin{array}{l}28 \mathrm{~g} / 8.4 \mathrm{MJ} \text { per } \mathrm{d} \\
\quad \text { almonds }\end{array}$ & $\begin{array}{l}\text { SBP NS } \\
\text { DBP NS }\end{array}$ & $\begin{array}{l}\text { NS-BP } \\
\text { NS-BP }\end{array}$ \\
\hline $\begin{array}{l}\text { Schutte et al. } \\
(2006)^{(155)}\end{array}$ & 8 weeks & $\begin{array}{l}n 62 \text { (control }=21, \\
\quad \text { walnut }=20, \text { cashew } \\
=21 \text { ) Met-S, } M \text { and } F, \\
45 \text { years }\end{array}$ & $\begin{array}{l}\text { RCT, parallel, } \\
\text { isoenergetic LF diet } \\
\text { (control) with/without } \\
\text { walnuts or cashews }\end{array}$ & $\begin{array}{l}63-103 \mathrm{~g} / \mathrm{d} \text { walnuts or } \\
\text { cashews }(20 \% \\
\text { energy) }\end{array}$ & $\begin{array}{l}\text { SBP NS } \\
\text { DBP NS }\end{array}$ & $\begin{array}{l}\text { NS-BP } \\
\text { NS-BP }\end{array}$ \\
\hline $\begin{array}{l}\text { Sheridan et al. } \\
(2007)^{(156)}\end{array}$ & $\begin{array}{l}4 \text { weeks per } \\
\text { arm }\end{array}$ & $\begin{array}{l}n 15, \text { hypercholestero- } \\
\text { laemic, } M \text { and F, } \\
\text { mean } 60 \text { (SD 12) years }\end{array}$ & $\begin{array}{l}\text { RCT, cross-over, } \\
\text { isoenergetic habitual } \\
\text { diet (control) with/ } \\
\text { without pistachios, } \\
\text { no washout }\end{array}$ & $\begin{array}{l}56-84 \mathrm{~g} / \mathrm{d} \text { pistachios } \\
\text { (15\% energy) }\end{array}$ & $\begin{array}{l}\text { SBP NS } \\
\text { DBP NS }\end{array}$ & $\begin{array}{l}\text { NS-BP; } 0 \cdot 1 \\
\text { NS-BP; } 0 \cdot 1\end{array}$ \\
\hline
\end{tabular}


fasting glucose or insulin ${ }^{(48,63-76)}$. One study that failed to achieve an improvement in insulin sensitivity supplemented participants' diets with $100 \mathrm{~g}$ almonds/d for 4 weeks. In this study there was a significant weight gain, which may have masked any benefit on insulin control ${ }^{(65)}$. Unexpected increases in plasma glucose (but not insulin) were observed with walnut and cashew consumption in women with polycystic ovary syndrome and adults with the metabolic syndrome, respectively ${ }^{(71,73)}$. Other studies investigated $\mathrm{HbA} 1 \mathrm{c}$ in individuals with type 2 diabetes and found that $28 \mathrm{~g}$ walnuts/ $\mathrm{d}$ and $36 \mathrm{~g}$ almonds/d reduced HbA1c by $4 \%{ }^{(62,64)}$. However, in other individuals, HbA1c did not change with $37-75 \mathrm{~g}$ mixed nuts/ $\mathrm{d}^{(66)}, 30-50 \mathrm{~g}$ walnuts/ $\mathrm{d}^{(69,70,72)}$ or $57-112 \mathrm{~g}$ almonds/d (for 4 weeks) ${ }^{(65)}$. The lack of effect in the latter study may have been due to the short intervention time. Whilst epidemiological studies suggest an association of nut consumption with improvement in glucoregulation and diabetes risk, not all evidence from randomised controlled trials is supportive. Some inconsistencies in findings may be attributed to variations in the number or health status of the study participants, length of trial, or the dose of nuts used.

Weighted mean changes in glucoregulation indicate significant reductions in fasting insulin and HOMA scores of 14 (95\% CI $-24,-4.5) \%$ and 34 (95\% CI - 49, - 19)\%, respectively, with small non-significant reductions of $2 \cdot 8$ $(95 \% \mathrm{CI}-6 \cdot 9,1 \cdot 3) \%$ and $1(95 \% \mathrm{CI}-3,0.9) \%$ for fasting glucose and HbA1c, respectively. This indicates positive effects of nut consumption on the most widely accepted markers of glucoregulation. Overall, there is considerable evidence of benefits of nut consumption for glycaemic control and insulin sensitivity observed after 4-6 weeks of consumption. However, inconsistencies make it difficult to reach precise conclusions on the role of nuts. The target population, dose and length of consumption (particularly to observe changes in HbA1c) need to be further considered so that targeted advice can be provided to consumers.

\section{Effects of nut consumption on blood pressure}

Studies measuring the effect of nut consumption on blood pressure are found in Table 4. Nuts consumed included walnuts, pistachios, groundnuts, almonds, cashews, hazelnuts, macadamias and mixed nuts in different forms including oil, whole nuts and nut flour added to baked goods. As with many studies using whole-food products, participant blinding was not possible. Amounts consumed ranged from 10 to $108 \mathrm{~g} / \mathrm{d}$ ( $\frac{1}{3}$ ounce to 4 ounces/d) (approximately $2-20 \%$ of energy intake). The length of consumption ranged from 3 weeks to 2 years. Whilst there were thirtysix intervention trials that reported on the effect of chronic consumption of nuts on blood pressure, most measured blood pressure as a secondary outcome. Comparisons were made with a healthy diet (sixteen studies), habitual diet (seven studies) or other food products including 
Table 5. Effects of nut consumption on inflammatory markers

\begin{tabular}{|c|c|c|c|c|c|c|c|}
\hline Author & Time & Subjects & Study design & Amount/type nuts & Measure & Outcomet & $\begin{array}{l}\text { Effect; } \\
\text { effect size }\end{array}$ \\
\hline \multicolumn{8}{|c|}{ Cross-sectional studies measuring effects of nut consumption on inflammatory markers (studies are presented in order of efficacy) } \\
\hline \multirow[t]{2}{*}{$\begin{array}{l}\text { Jiang et al. } \\
(2006)^{(90)}\end{array}$} & \multirow[t]{2}{*}{ X-sect } & \multirow{2}{*}{$\begin{array}{l}n 6080, \text { healthy, } \mathrm{M} \text { and } \mathrm{F}, \\
\text { mean } 62 \text { (range } 45-84 \text { ) } \\
\text { years }\end{array}$} & \multirow[t]{2}{*}{$\begin{array}{l}\text { X-sect, FFQ and inflamma- } \\
\text { tory markers, MESA }\end{array}$} & \multirow[t]{2}{*}{$\begin{array}{l}\text { Rare to }>4 \text { serves/week } \\
\text { any nuts and seeds }\end{array}$} & CRP & $\begin{array}{l}\text { Negative correlation }{ }^{\star *} \\
r 0.06\end{array}$ & + \\
\hline & & & & & $\mathrm{IL}-6$ & $\begin{array}{l}\text { Negative correlation }{ }^{\star *} \\
r 0.05\end{array}$ & + \\
\hline \multirow{4}{*}{$\begin{array}{l}\text { Salas-Salvadó } \\
\text { et al. } \\
(2008)^{(91)}\end{array}$} & \multirow[t]{4}{*}{$X$-sect } & \multirow{4}{*}{$\begin{array}{l}n 772, \text { high risk of CVD, } \mathrm{M} \\
\text { and } \mathrm{F} \text {, mean } 68 \\
\text { (range } 55-80 \text { ) years }\end{array}$} & \multirow{4}{*}{$\begin{array}{l}\text { X-sect, FFQ and inflamma- } \\
\text { tory markers (PREDIMED } \\
\text { trial) }\end{array}$} & \multirow[t]{2}{*}{$\begin{array}{c}\text { Tertiles (any nuts) } \\
\mathrm{T} 1<3.92 \mathrm{~g}\end{array}$} & ICAM & $\downarrow 17 \%^{*}$ & + \\
\hline & & & & & VCAM & NS & NS-I \\
\hline & & & & T2 $3.92-10.84 \mathrm{~g}$ & IL-6 & NS & NS-I \\
\hline & & & & & CRP & NS & NS-I \\
\hline \multirow{2}{*}{$\begin{array}{l}\text { O'Neil et al. } \\
(2011)^{(49)}\end{array}$} & \multirow[t]{2}{*}{ X-sect } & \multirow{3}{*}{$\begin{array}{l}n 13292 \text {, general population, } \\
M \text { and F, mean } 57 \text { years } \\
n 6309, \mathrm{~F}, \mathrm{Dm}\end{array}$} & \multirow{2}{*}{$\begin{array}{l}\text { 1999-2004 NHANES, } 24 \mathrm{~h} \\
\text { recall and CRP }\end{array}$} & \multirow{4}{*}{$\begin{array}{l}\text { All nuts } \leq 7 \mathrm{~g} / \mathrm{d} \\
\text { Tree nuts } 7 \mathrm{~g} / \mathrm{d} \\
0->5 \text { serves/week; } 1 \\
\text { serve }=28 \mathrm{~g} \text { any nuts }\end{array}$} & CRP & NS & NS-I \\
\hline & & & & & CRP & $\downarrow 12 \%^{*}$ & + \\
\hline $\begin{array}{l}\text { Li et al. } \\
(2009)^{(92)}\end{array}$ & X-sect & & $\begin{array}{l}\text { FFQ and inflammatory } \\
\text { markers }\end{array}$ & & ICAM & NS & NS-I \\
\hline & & & & & E-selectin & NS & NS-I \\
\hline \multicolumn{8}{|c|}{ Chronic effects of nuts on inflammatory markers (studies are presented in order of efficacy) } \\
\hline \multirow{6}{*}{$\begin{array}{l}\text { Zhao et al. } \\
(2004)^{(93)}\end{array}$} & \multirow{6}{*}{$\begin{array}{l}6 \text { weeks } \\
\text { per arm }\end{array}$} & \multirow{6}{*}{$\begin{array}{l}n 23, \text { hypercholesterolaemic, } \\
\mathrm{M} \text { and } \mathrm{F} \text {, mean } 50 \text { (SD 2) } \\
\text { years }\end{array}$} & $\mathrm{RCT}$, cross-over, LA diet & \multirow{6}{*}{$\begin{array}{l}37 \mathrm{~g} / \mathrm{d} \text { walnuts }+15 \mathrm{~g} / \mathrm{d} \\
\text { walnut oil }\end{array}$} & CRP (ALA) & $\downarrow 75 \%^{*}$ & + \\
\hline & & & (walnut + walnut oil) $v$. & & VCAM (ALA) & $\downarrow 12 \%^{*}$ & + \\
\hline & & & ALA diet (flax + walnut + & & E-selectin (ALA) & $\downarrow 12 \%^{*}$ & + \\
\hline & & & walnut oil) v. American & & E-selectin (LA) & $\downarrow 7 \%$ & + \\
\hline & & & diet (control), no washout, & & $\operatorname{CRP}(\mathrm{LA})$ & $\downarrow 35 \%$ & + \\
\hline & & & isoenergetic & & VCAM (LA) & $\downarrow 7 \%$ & + \\
\hline \multirow{6}{*}{$\begin{array}{l}\text { Mena et al. } \\
(2009)^{(84)}\end{array}$} & \multirow[t]{6}{*}{3 months } & \multirow{6}{*}{$\begin{array}{l}n 106 \text { (control }=36, O O \\
\quad=45, \text { nuts }=35), \text { high } \\
\text { risk of CVD, M and } F \text {, } \\
\text { mean } 68 \text { (SD 8) years }\end{array}$} & RCT, parallel, Med diet + & \multirow[t]{6}{*}{$30 \mathrm{~g} / \mathrm{d}$ mixed nuts $\ddagger$} & IL-6 & $\downarrow 40 \%^{*}$ & $+; 0.3$ \\
\hline & & & $O O v$. Med diet + nuts $v$ & & ICAM & $\downarrow 52 \%^{*}$ & $+; 1 \cdot 1$ \\
\hline & & & LF diet (control) & & VCAM & $\downarrow 33 \%^{*}$ & $+; 1.5$ \\
\hline & & & (PREDIMED) & & CRP & $\downarrow 78 \%{ }^{*}$ & $+; 0.4$ \\
\hline & & & & & E-selectin & NS & NS-I; $-0 \cdot 2$ \\
\hline & & & & & P-selectin & NS & NS-I; 0.2 \\
\hline Esposito et al. & 2 years & $n 180$ (control $=90$, Med & RCT, parallel, Med diet & $20-50 \mathrm{~g} / \mathrm{d}$ walnuts & CRP & $\downarrow 36 \%^{*}$ & + \\
\hline$(2004)^{(47)}$ & & $\operatorname{diet}=90)$, Met-S, M and & (including walnuts) $v$. & & IL-6 & $\downarrow 28 \%^{*}$ & + \\
\hline & & $F$, mean 44 (SD 6) years & prudent diet (control) & & $\mathrm{IL}-7$ & $\downarrow 21 \%^{*}$ & + \\
\hline & & & & & IL-18 & $\downarrow 9 \%^{*}$ & + \\
\hline Solà et al. & 4 weeks & $n 113$ (control $=28$, nut & $\mathrm{RCT}$, parallel, isoenergetic & $30 \mathrm{~g} / \mathrm{d}$ hazelnuts & VCAM (A) (B) & NS & NS-I; 0.1 \\
\hline$(2012)^{(86)}$ & per arm & $=28$, nut + sterol $=30$ & cocoa + hazelnut $(\mathrm{A}) \mathrm{v}$ & & VCAM (C) & NS & NS-I; $0 \cdot 0$ \\
\hline & & nut + sterol + fibre & cocoa + hazelnut + & & ICAM (A) (B) (C) & NS & NS-I; 0.0 \\
\hline & & $=27)$, risk of $\mathrm{CVD}, \mathrm{M}$ and & phytosterol (B) v. cocoa + & & IL-6 (A) (B) (C) & NS & NS-I \\
\hline & & $F$, mean 54 (SD 9) years & hazelnut + phytosterol + & & $\operatorname{CRP}(A)(B)$ & & NS-I \\
\hline & & & $\begin{array}{l}\text { soluble fibre (C) } v \text {. cocoa } \\
\text { (control), no washout }\end{array}$ & & $\operatorname{CRP}(\mathrm{C})$ & $\downarrow 33 \%^{*}$ & NS-I \\
\hline $\begin{array}{l}\text { Sari et al. } \\
(2010)^{(59)}\end{array}$ & $\begin{array}{l}4 \text { weeks } \\
\text { per arm }\end{array}$ & $\begin{array}{l}n 32, \text { healthy, M, mean } 22 \\
\text { (range } 21-24 \text { ) years }\end{array}$ & $\begin{array}{l}\text { Prospective cohort, iso- } \\
\text { energetic Med diet }\end{array}$ & $80-100 \mathrm{~g} / \mathrm{d}$ pistachios & $\begin{array}{l}\text { IL-6 } \\
\text { CRP }\end{array}$ & $\begin{array}{l}\downarrow \\
\text { NS }\end{array}$ & $\begin{array}{l}+; 0.4 \\
\text { NS-I }\end{array}$ \\
\hline & & & $\begin{array}{l}\text { (control) with/without } \\
\text { pistachios, no washout }\end{array}$ & & $\mathrm{TNF} \alpha$ & NS & NS-I; 0.0 \\
\hline $\begin{array}{l}\text { Jenkins et al. } \\
(2002)^{(99)}\end{array}$ & $\begin{array}{l}4 \text { weeks } \\
\text { per arm }\end{array}$ & $\begin{array}{l}n 13, \mathrm{M} \text { and } \mathrm{F} \text {, mean } 65 \\
\text { (sD 3) years, hyper- } \\
\text { lipidaemic }\end{array}$ & $\begin{array}{l}\text { Prospective cohort, LF diet } \\
\text { (control) and portfolio diet } \\
\text { (soya/sterol/fibre/nuts) }\end{array}$ & $28 \mathrm{~g} / \mathrm{d}$ almonds & CRP & $\downarrow 26 \%$ & + \\
\hline $\begin{array}{l}\text { Jenkins et al. } \\
(2003)^{(89)}\end{array}$ & 4 weeks & $\begin{array}{l}n 46 \text { (control }=16 \text {, statin } \\
=14, \text { nut }=16 \text { ), hyper- } \\
\text { lipidaemic, } M \text { and } F \text {, mean } \\
59 \text { (SD 1) years }\end{array}$ & $\begin{array}{l}\text { RCT, parallel, isoenergetic } \\
\text { statin } v \text {. sterol + soya + } \\
\text { almonds v. LF diet } \\
\text { (control) }\end{array}$ & $14 \mathrm{~g} / 4.2 \mathrm{MJ}$ almonds per $\mathrm{d}$ & CRP & $\downarrow 18 \%{ }^{*}$ & $+; 0.1$ \\
\hline $\begin{array}{l}\text { Canales et al. } \\
(2011)^{(97)}\end{array}$ & $\begin{array}{l}5 \text { weeks } \\
\text { per arm }\end{array}$ & $\begin{array}{l}n \text { 22, risk of CVD, } M \text { and } F \text {, } \\
\text { mean } 55(\mathrm{SD} 2) \text { years }\end{array}$ & $\begin{array}{l}\text { RCT, cross-over, meat pro- } \\
\text { duct (control) with/without } \\
\text { walnuts, } 5 \text { weeks washout }\end{array}$ & $21 \mathrm{~g} / \mathrm{d}$ walnuts & $\begin{array}{l}\text { VCAM } \\
\text { ICAM }\end{array}$ & $\begin{array}{l}\downarrow 12 \%^{*} \\
\downarrow 17 \%^{*}\end{array}$ & $\begin{array}{l}+; 0.4 \\
+; 0.3\end{array}$ \\
\hline $\begin{array}{l}\text { Jenkins et al. } \\
(2005)^{(94)}\end{array}$ & $\begin{array}{l}1 \text { month } \\
\text { per arm }\end{array}$ & $\begin{array}{l}n 34 \text {, hyperlipidaemic, M and } \\
\mathrm{F} \text {, mean } 55 \text { years (SD 7) } \\
\text { years }\end{array}$ & $\begin{array}{l}\text { RCT, cross-over, iso- } \\
\text { energetic statin } v \text {. phyto- } \\
\text { sterol + soya + almonds } \\
v \text {. LF diet (control), } 4 \\
\text { weeks washout }\end{array}$ & $14 \mathrm{~g} / 4 \cdot 2 \mathrm{MJ}$ almonds & $\begin{array}{l}\text { CRP (all subjects) } \\
\text { CRP (subjects } \leq 3.5 \mathrm{mg} / \mathrm{l})\end{array}$ & $\begin{array}{l}\text { NS } \\
\downarrow 9 \%\end{array}$ & $\begin{array}{l}\text { NS-I; } 0.0 \\
+; 0.4\end{array}$ \\
\hline
\end{tabular}




\section{镸彭 Nutrition Research Reviews}

Table 5. Continued

\begin{tabular}{|c|c|c|c|c|c|c|c|}
\hline Author & Time & Subjects & Study design & Amount/type nuts & Measure & Outcome† & $\begin{array}{l}\text { Effect; } \\
\text { effect size }\end{array}$ \\
\hline $\begin{array}{l}\text { Rajaram et al. } \\
(2010)^{(95)}\end{array}$ & $\begin{array}{l}4 \text { weeks } \\
\text { per arm }\end{array}$ & $\begin{array}{l}n 25 \text {, healthy, } M \text { and } F \text {, } \\
\text { mean } 41 \text { (SD 13) years }\end{array}$ & $\begin{array}{l}\text { RCT, cross-over, low-almond } \\
\text { diet }(\mathrm{L}) v \text {. high-almond } \\
\text { diet }(\mathrm{H}) v \text {. healthy heart } \\
\text { diet (control), no washout }\end{array}$ & $\begin{array}{l}\text { Low almond (L) (34 g/8.4 MJ } \\
\text { per d) } \\
\text { High almond }(\mathrm{H}) \\
(68 \mathrm{~g} / 8.4 \mathrm{MJ} \text { per d })\end{array}$ & $\begin{array}{l}\text { E-selectin (H) } \\
\text { E-selectin (L) } \\
\text { CRP (H) } \\
\text { CRP (L) } \\
\text { IL-6 (H) (L) }\end{array}$ & $\begin{array}{l}\downarrow 8 \% * \\
\text { NS } \\
\downarrow 5 \%^{*} \\
\downarrow 9 \%^{*} \\
\text { NS }\end{array}$ & $\begin{array}{l}+; 0.5 \\
\text { NS-I; } 0.0 \\
+; 0 \cdot 1 \\
+; 0.1 \\
\text { NS-I; } 0.1\end{array}$ \\
\hline $\begin{array}{l}\text { Ros et al. } \\
(2004)^{(98)}\end{array}$ & $\begin{array}{l}4 \text { weeks } \\
\text { per arm }\end{array}$ & $\begin{array}{l}n 20, \mathrm{M} \text { and } \mathrm{F} \text {, hypercholes- } \\
\text { terolaemic, mean } 55 \\
\text { (range } 26-75 \text { ) years }\end{array}$ & $\begin{array}{l}\text { RCT, cross-over, } \\
\text { isoenergetic Med diet } \\
\text { (control) with/without nuts, } \\
\text { no washout }\end{array}$ & $\begin{array}{l}\text { About } 65 \mathrm{~g} / \mathrm{d} \text { walnuts } \\
(32 \% \text { energy })\end{array}$ & $\begin{array}{l}\text { VCAM } \\
\text { ICAM } \\
\text { CRP }\end{array}$ & $\begin{array}{l}\downarrow 12 \% * \\
\text { NS } \\
\text { NS }\end{array}$ & $\begin{array}{l}+; 0.5 \\
\text { NS-I; } 0.3 \\
\text { NS-I; } 0.0\end{array}$ \\
\hline $\begin{array}{l}\text { Estruch et al. } \\
(2006)^{(58)}\end{array}$ & 3 months & $\begin{array}{l}n 772 \text { (control }=257,00 \\
=257, \text { nut }=258 \text { ), high } \\
\text { risk of CVD, M and F, } \\
\text { mean } 69 \text { (SD 6) years }\end{array}$ & $\begin{array}{l}\text { RCT, parallel, Med diet }+ \\
\text { OO v. Med diet }+ \text { nuts } \\
\text { v. LF diet (control) } \\
\text { (PREDIMED) }\end{array}$ & $30 \mathrm{~g} / \mathrm{d}$ mixed nuts $\ddagger$ & $\begin{array}{l}\text { CRP } \\
\text { ICAM } \\
\text { VCAM } \\
\text { IL-6 }\end{array}$ & $\begin{array}{l}\text { NS } \\
\text { Data N/A* } \\
\text { Data N/A* } \\
\text { Data N/A* }\end{array}$ & $\begin{array}{l}\text { NS-I } \\
+ \\
+ \\
+\end{array}$ \\
\hline $\begin{array}{l}\text { Chiang et al. } \\
(2012)^{(96)}\end{array}$ & $\begin{array}{l}4 \text { weeks } \\
\text { per arm }\end{array}$ & $\begin{array}{l}n 25 \text {, hyperlipidaemic, } M \text { and } \\
\mathrm{F} \text {, mean } 33 \text { (range } \\
23-65 \text { ) years }\end{array}$ & $\begin{array}{l}\text { RCT, cross-over, } \\
\text { isoenergetic fatty fish } v \text {. } \\
\text { walnuts } v \text {. no nut/fish } \\
\text { (control) }\end{array}$ & $43 \mathrm{~g} / 10 \mathrm{MJ}$ walnuts per $\mathrm{d}$ & $\begin{array}{l}\text { CRP } \\
\text { ICAM } \\
\text { TNF- } \alpha \\
\text { IL-1 } \beta \text { and IL-6 } \\
\text { E-selectin }\end{array}$ & $\begin{array}{l}\text { NS } \\
\text { NS } \\
\text { NS } \\
\text { NS } \\
\text { NS v. control } \\
(\downarrow v \text {. fish* }\end{array}$ & $\begin{array}{l}\text { NS-I } \\
\text { NS-I } \\
\text { NS-I } \\
\text { NS-I } \\
\text { NS-I } \\
+\end{array}$ \\
\hline $\begin{array}{l}\text { Kasim-Kara- } \\
\text { kas et al. } \\
(2004)^{(73)}\end{array}$ & 3 months & $\begin{array}{l}n \text { 17, mean } 34 \text { (SD 5) years, } \\
\text { F, PCOS }\end{array}$ & $\begin{array}{l}\text { Prospective cohorts } \\
\text { isoenergetic habitual diet } \\
\text { (control) } v \text {. walnuts }\end{array}$ & $106 \mathrm{~g} / \mathrm{d}$ walnuts & TNF- $\alpha$ & NS & NS-I; $1 \cdot 0$ \\
\hline $\begin{array}{l}\text { Jenkins et al. } \\
(2011)^{(66)}\end{array}$ & 3 months & $\begin{array}{l}n 117(\text { control }=37 \\
37 \mathrm{~g} / \mathrm{d}=40,75 \mathrm{~g} / \mathrm{d}=40) \\
\text { Dm, M and } \mathrm{F}, \text { mean } 62 \\
(\mathrm{SD} 10) \text { years }\end{array}$ & $\begin{array}{l}\mathrm{RCT} \text {, parallel, } 75 \mathrm{~g} / \mathrm{d} \text { nuts } v \text {. } \\
37 \mathrm{~g} / \mathrm{d} \text { nuts + half muffin } \\
v . \text { muffin (control) }\end{array}$ & 75 or $37 \mathrm{~g} / \mathrm{d}$ mixed nuts§ & $\begin{array}{l}\text { CRP (full dose) } \\
\text { CRP (half dose) }\end{array}$ & $\begin{array}{l}\text { NS } \\
\text { NS }\end{array}$ & $\begin{array}{l}\text { NS-I; } 0 \cdot 1 \\
\text { NS-I; } 0 \cdot 2\end{array}$ \\
\hline $\begin{array}{l}\text { Jenkins et al. } \\
(2002)^{(99)}\end{array}$ & $\begin{array}{l}1 \text { month } \\
\text { per arm }\end{array}$ & $\begin{array}{l}n 27 \text {, hyperlipidaemic, } M \text { and } \\
F, \text { mean } 64 \text { (SD 9) years }\end{array}$ & $\begin{array}{l}\text { RCT, cross-over almonds } v \text {. } \\
\text { half almonds }+ \text { half muffin } \\
v \text {. muffin (control) }\end{array}$ & $\begin{array}{l}73 \mathrm{~g} / \mathrm{d} \text { almonds or } 36 \mathrm{~g} / \mathrm{d} \\
\text { almonds }\end{array}$ & CRP & NS & NS-I; 0.0 \\
\hline $\begin{array}{l}\text { Adamsson } \\
\text { et al. } \\
(2011)^{(46)}\end{array}$ & 4 weeks & $\begin{array}{l}n 86 \text { (control }=42, \text { NOR- } \\
\text { DIET = 44), hypercholes- } \\
\text { terolaemic, M and F, } \\
\text { mean } 53 \text { (SD 8) years }\end{array}$ & $\begin{array}{l}\text { RCT, parallel, NORDIET } \\
\text { (fibre, fish, LF dairy, nuts) } \\
v \text {. habitual diet (control) }\end{array}$ & Almonds ad libitum & CRP & NS & NS-I; 0.1 \\
\hline $\begin{array}{l}\text { Thomazella } \\
\text { et al. } \\
(2011)^{(48)}\end{array}$ & 3 months & $\begin{array}{l}n 40(\text { control }=19, \text { Med } \\
\text { diet }=21), M, C V D \\
\text { mean } 55(\text { SD } 5) \text { years }\end{array}$ & $\begin{array}{l}\text { Prospective controlled study, } \\
\text { Med diet (including nuts) } \\
\text { v. LF diet (control) }\end{array}$ & $10 \mathrm{~g} / \mathrm{d}$ any nuts & $\begin{array}{l}\text { CRP } \\
\text { ICAM } \\
\text { VCAM }\end{array}$ & $\begin{array}{l}\text { NS } \\
\text { NS } \\
\text { NS }\end{array}$ & $\begin{array}{l}\text { NS-I; } 0.3 \\
\text { NS-I; } 0.3 \\
\text { NS-I; } 0.0\end{array}$ \\
\hline $\begin{array}{l}\text { López-Uriarte } \\
\text { et al. } \\
(2010)^{(106)}\end{array}$ & 12 weeks & $\begin{array}{l}n 50 \text { (control }=25, \text { nut } \\
\quad=25), \text { Met-S, M and F, } \\
\text { mean } 5 \text { (SD 8) years }\end{array}$ & $\begin{array}{l}\text { RCT, parallel, American } \\
\text { Heart Association diet } \\
\text { (control) with/without nuts, } \\
\text { not isoenergetic }\end{array}$ & $30 \mathrm{~g} / \mathrm{d}$ mixed nuts $\neq$ & $\begin{array}{l}\text { ICAM } \\
\text { VCAM }\end{array}$ & $\begin{array}{l}\text { NS } \\
\text { NS }\end{array}$ & $\begin{array}{l}\text { NS-I; } 0.3 \\
\text { NS-I; } 0.6\end{array}$ \\
\hline $\begin{array}{l}\text { Damasceno } \\
\text { et al. } \\
(2011)^{(149)}\end{array}$ & $\begin{array}{l}4 \text { weeks } \\
\text { per arm }\end{array}$ & $\begin{array}{l}n 18, \mathrm{M} \text { and } \mathrm{F}, \text { mean } 56 \\
\text { (sD 13) years, hyper- } \\
\text { cholesterolaemic }\end{array}$ & $\begin{array}{l}\text { RCT, cross-over, iso- } \\
\text { energetic Med diet }+ \\
\text { walnuts (W) } v \text {. almonds } \\
\text { (A) } v \text {. OO (control), no } \\
\text { washout }\end{array}$ & $\begin{array}{l}40-65 \mathrm{~g} / \mathrm{d} \text { walnuts }(\mathrm{W}) \\
50-75 \mathrm{~g} / \mathrm{d} \text { almonds (A) }\end{array}$ & $\begin{array}{l}\operatorname{VCAM}(W \text { and } A) \\
\text { ICAM }(W \text { and } A) \\
\text { CRP }(W \text { and } A)\end{array}$ & $\begin{array}{l}\text { NS } \\
\text { NS } \\
\text { NS }\end{array}$ & $\begin{array}{l}\text { NS-I; }-0.2 \\
\text { NS-I; } 0.2 \\
\text { NS-I; } 0.2\end{array}$ \\
\hline $\begin{array}{l}\text { Kalgaonkar } \\
\text { et al. } \\
(2011)^{(62)}\end{array}$ & 6 weeks & $\begin{array}{l}n 31 \text { (almond }=14, \text { walnut } \\
=17), F, P C O S, \text { age } \\
\text { range } 20-45 \text { years }\end{array}$ & $\begin{array}{l}\text { Pre-/post-measures, walnuts } \\
\text { v. almonds }\end{array}$ & $\begin{array}{l}36 \mathrm{~g} / \mathrm{d} \text { walnuts }(W), 26 \mathrm{~g} / \mathrm{d} \\
\text { almonds }(A)\end{array}$ & $\begin{array}{l}\text { IL-6 }(\mathrm{W} v \cdot \mathrm{v} \cdot \mathrm{A}) \\
\text { TNF- } \alpha(\mathrm{W} v . \mathrm{A}) \\
\mathrm{IL}-1 \beta(\mathrm{W} v \cdot \mathrm{v}) \\
\operatorname{CRP}(\mathrm{W} v \cdot \mathrm{A})\end{array}$ & $\begin{array}{l}\text { NS } \\
\text { NS } \\
\text { NS } \\
\text { NS }\end{array}$ & $\begin{array}{l}\text { NS-l; } 1 \cdot 3 \\
\text { NS-I; } 1.2 \\
\text { NS-I; } 0.9 \\
\text { NS-I; } 0.4\end{array}$ \\
\hline $\begin{array}{l}\text { Kurlandsky \& } \\
\text { Stot } \\
(2006)^{(157)}\end{array}$ & 6 weeks & $\begin{array}{l}n 41 \text { (control }=10, \text { choco- } \\
\text { late }=10, \text { nut }=10, \text { nut } \\
+ \text { chocolate }=11), \\
\text { healthy, } F, \text { mean } 47 \\
\text { (SD } 9) \text { years }\end{array}$ & $\begin{array}{l}\text { RCT, parallel, LF diet } \\
\text { (control) v. chocolate } v \text {. } \\
\text { almond (A) v. chocolate }+ \\
\text { almonds (CA) }\end{array}$ & $60 \mathrm{~g} / \mathrm{d}$ almonds & $\begin{array}{l}\text { CRP (CA) } \\
\text { ICAM (CA) } \\
\operatorname{VCAM~(CA)~} \\
\text { CRP }(A) \\
\operatorname{VCAM}(A) \\
\operatorname{ICAM}(A)\end{array}$ & $\begin{array}{l}\text { NS } \\
\text { NS } \\
\text { NS } \\
\text { NS } \\
\text { NS } \\
\text { NS }\end{array}$ & $\begin{array}{l}\text { NS-I; } 0 \cdot 0 \\
\text { NS-I; } 0 \cdot 2 \\
\text { NS-I; } 0 \cdot 2 \\
\text { NS-I; } 0 \cdot 2 \\
\text { NS-I; } 0 \cdot 1 \\
\text { NS-I; } 0 \cdot 2\end{array}$ \\
\hline
\end{tabular}




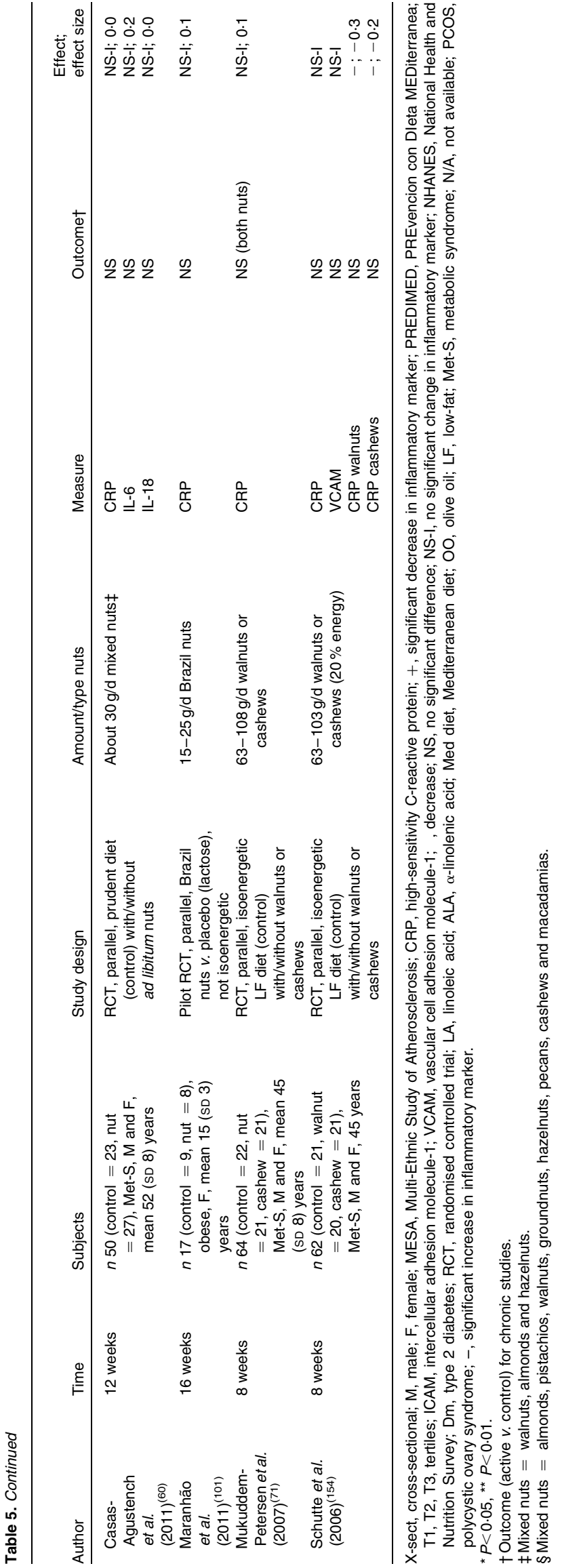

butter, muffins, processed meat, olive oil and cocoa. Of the studies, four compared habitual or healthy diets with intervention diets including nuts NORDIET $^{(46)}$ or a Mediterranean diet ${ }^{(47,48)}$; only one of the studies reported controlling for salt intake ${ }^{(46)}$. The remaining studies used control diets with unsalted nuts added as the intervention but overall dietary salt intake was not specified. Four prospective cohort studies measured blood pressure or incidence of hypertension in participants consuming nuts. The Physicians' Study demonstrated a significant reduction in self-reported hypertension after 12 months in those consuming nuts $\geq$ twice per week (hazard ratio 0.87; $95 \%$ CI $0.79,0.96)$ and greatest reduction with consumption $\geq 7$ times per week (hazard ratio $0 \cdot 77 ; 95 \%$ CI $0.64,0.93)^{(77)}$. However, salt intake and changes in weight were not accounted for, which could have affected outcomes observed. The Coronary Artery Risk Development in Young Adults (CARDIA) Study demonstrated an inverse relationship between nut consumption and prevalence of hypertension despite those classified as the highest consumers only consuming nuts $\geq 2$ times per week (hazard ratio $0.85 ; 95 \%$ CI $0.64,0.93)^{(78)}$. In support of this, the Atherosclerosis Risk in Communities (ARIC) Study also reported that nut consumption was inversely related to a reduced risk of hypertension; those who consumed approximately two serves of nuts per week were at a lower risk of hypertension than those who rarely or never consumed nuts (hazard ratio 0.87; $95 \%$ CI 0.77 , $0 \cdot 97)^{(79)}$. In contrast, the SUN Study demonstrated no association between hypertension and nut consumption after a 4 -year follow-up ${ }^{(80)}$. However, the young educated adult sample in this study is less likely to demonstrate improvements in blood pressure with a dietary intervention than older individuals who are more likely to have higher blood pressure.

In all, four cross-sectional studies were identified comparing blood pressure or prevalence of hypertension in nut consumers with low-/non-nut consumers. The National Health and Nutrition Survey (NHANES) observed a general population and found a $3 \%$ lower risk of hypertension and $1 \mathrm{mmHg}$ reduction in systolic and diastolic blood pressure in nut consumers ${ }^{(49)}$. The Canary Nutrition Survey demonstrated a trend for reduced prevalence of hypertension with higher nut consumption but this did not reach significance $^{(50)}$. The Multi-Ethnic Study of Atherosclerosis (MESA) in Spain did not find an association with a healthy dietary pattern (incorporating an undetermined quantity of nuts) and blood pressure ${ }^{(52)}$. The authors suggest that routinely assessed blood pressure may have increased risk factor awareness, thereby attenuating associations with dietary intake. No association was found with hypertension and nut consumption in participants with a high risk of $\mathrm{CVD}^{(81)}$. However, $90 \%$ of the participants were hypertensive which may have made it difficult to demonstrate a relationship in this population. It is more difficult to account for health benefits from an individual food with 
Table 6. Chronic effect of nut consumption on endothelial vasodilator function (studies are presented in order of efficacy) $\dagger$

\begin{tabular}{|c|c|c|c|c|c|c|}
\hline Author & Time & Subjects & Study design & Amount/type nuts & $\begin{array}{l}\text { Outcome } \\
\text { (active } \\
\text { V. control) }\end{array}$ & $\begin{array}{l}\text { Effect; } \\
\text { effect size }\end{array}$ \\
\hline $\begin{array}{l}\text { Ros et al. } \\
\qquad(2004)^{(98)}\end{array}$ & $\begin{array}{l}4 \text { weeks } \\
\text { per arm }\end{array}$ & $\begin{array}{l}n 20, \text { hypercholesterolaemic, } M \\
\text { and } \mathrm{F} \text {, mean } 55 \text { (range } \\
26-75 \text { ) years }\end{array}$ & $\begin{array}{l}\text { RCT, cross-over, isoenergetic Med diet } \\
\text { (control) with/without walnuts, no wash- } \\
\text { out }\end{array}$ & About $65 \mathrm{~g} / \mathrm{d}$ walnuts (32\% energy) & $\uparrow 64 \% *$ & $+; 0.3$ \\
\hline $\begin{array}{l}\text { Ma et al. } \\
(2010)^{(72)}\end{array}$ & $\begin{array}{l}8 \text { weeks } \\
\text { per arm }\end{array}$ & $\begin{array}{l}n 21, \mathrm{Dm}, \mathrm{M} \text { and } \mathrm{F}, \text { mean } 58 \\
\quad(\mathrm{SD} 8) \text { years }\end{array}$ & $\begin{array}{l}\text { RCT, cross-over, ad libitum diet (control) } \\
\text { with/without walnuts, } 8 \text { weeks washout }\end{array}$ & $56 \mathrm{~g} / \mathrm{d}$ walnuts & $\uparrow 45 \% \%^{*}$ & $+; 0.6$ \\
\hline $\begin{array}{l}\text { West et al. } \\
(2010)^{(104)}\end{array}$ & $\begin{array}{l}6 \text { weeks } \\
\text { per arm }\end{array}$ & $\begin{array}{l}n 20 \text {, hypercholesterolaemic, } M \\
\text { and } F \text {, mean } 49 \text { (SD 6) years }\end{array}$ & $\begin{array}{l}\text { RCT, cross-over, American diet (control) } v \text {. } \\
\text { LA diet (flax }+ \text { walnut }+ \text { walnut oil) } v \text {. } \\
\text { ALA diet (walnut }+ \text { walnut oil), } \\
\text { no washout }\end{array}$ & $37 \mathrm{~g} / \mathrm{d}$ walnuts $+15 \mathrm{~g} / \mathrm{d}$ walnut oil & ALA $\uparrow 34 \% *$ & $+; 0.4$ \\
\hline $\begin{array}{l}\text { Sari et al. } \\
\quad(2010)^{(59)}\end{array}$ & $\begin{array}{l}4 \text { weeks } \\
\text { per arm }\end{array}$ & $\begin{array}{l}n 32, \text { healthy, } M, \text { mean } 22 \\
\text { (range } 21-24) \text { years }\end{array}$ & $\begin{array}{l}\text { RCT, cross-over, isoenergetic Med diet } \\
\text { (control) with/without pistachios, no } \\
\text { washout }\end{array}$ & $80-100 \mathrm{~g} / \mathrm{d}$ pistachios (20\% energy) & $\begin{array}{l}\text { LA NS } \\
\uparrow 24 \%^{*}\end{array}$ & $\begin{array}{l}\text { NS-EF; } 0 \cdot 1 \\
+; 1 \cdot 0\end{array}$ \\
\hline $\begin{array}{l}\text { Esposito et al. } \\
\qquad(2010)^{(47)}\end{array}$ & 2 years & $\begin{array}{l}n 180 \text { (control }=90, \text { Med diet } \\
\quad=90), \text { Met-S, M and F, mean } \\
44 \text { (SD 6) years }\end{array}$ & $\begin{array}{l}\text { RCT, parallel, Med diet (including walnuts) } \\
\text { v. prudent diet (control) }\end{array}$ & $20-50 \mathrm{~g} / \mathrm{d}$ walnuts & $\uparrow 21 \% *$ & $+; 0.9$ \\
\hline $\begin{array}{l}\text { Mercanligil et al. } \\
\qquad(2007)^{(74)}\end{array}$ & $\begin{array}{l}\text { 4-week } \\
\text { period }\end{array}$ & $\begin{array}{l}n 15, \mathrm{M}, \text { mean } 48 \text { (SD } 8 \text { ) years, } \\
\text { hypercholesterolaemic }\end{array}$ & $\begin{array}{l}\text { Two-period study, LF diet (control) v. LF + } \\
\text { hazelnuts, non-isoenergetic }\end{array}$ & $40 \mathrm{~g} / \mathrm{d}$ hazelnuts ( $12 \%$ energy) & NS & NS-EF \\
\hline $\begin{array}{l}\text { López-Uriarte } \\
\text { et al. } \\
(2010)^{(106)}\end{array}$ & $\begin{array}{l}12 \text { weeks } \\
\text { per arm }\end{array}$ & $\begin{array}{l}n 50(\text { control }=25, \text { nut }=25) \\
\text { Met-S, M and F, mean } 52 \\
(\text { SD 8) years }\end{array}$ & $\begin{array}{l}\text { RCT, parallel, American Heart Association } \\
\text { diet (control) (mixed nuts, not iso- } \\
\text { energetic) }\end{array}$ & $30 \mathrm{~g} / \mathrm{d}$ mixed nuts $\ddagger$ & NS & NS-EF; 0.0 \\
\hline $\begin{array}{l}\text { Thomazella et al. } \\
\qquad(2011)^{(48)}\end{array}$ & 3 months & $\begin{array}{l}n 40 \text { (control }=19, \text { Med diet } \\
\quad=21), M, C V D, \text { mean } 55 \\
\text { (SD 5) years }\end{array}$ & $\begin{array}{l}\text { Prospective controlled study, Med diet } \\
\text { (including nuts) v. LF diet (control) }\end{array}$ & $10 \mathrm{~g} / \mathrm{d}$ any nuts & NS & NS-EF; 0.0 \\
\hline \multirow[t]{2}{*}{$\begin{array}{l}\text { West et al. } \\
\qquad(2012)^{(107)}\end{array}$} & $\begin{array}{l}4 \text { weeks } \\
\text { per arm }\end{array}$ & $\begin{array}{l}n 25 \text {, hypercholesterolaemic, } M \\
\text { and } \mathrm{F} \text {, mean } 48 \text { (SD 2) years }\end{array}$ & $\begin{array}{l}\text { RCT, cross-over, LF diet (control) } v \text {. low- } \\
\text { dose pistachios } v \text {. high-dose pistachios, } \\
\text { no washout }\end{array}$ & $32-63 \mathrm{~g} / \mathrm{d}$ pistachios & NS & NS-EF; $-0 \cdot 10$ \\
\hline & & & & $63-126 \mathrm{~g} / \mathrm{d}$ pistachios & NS & NS-EF; $0 \cdot 10$ \\
\hline
\end{tabular}

M, male; F, female; RCT, randomised controlled trial; Med diet, Mediterranean diet; $\uparrow$, increase; +, significant increase in endothelial function; Dm, type 2 diabetes mellitus; LA, linoleic acid; ALA, $\alpha$-linolenic acid; NS, not significant; NS-EF, no significant change in endothelial function; Met-S, metabolic syndrome; LF, low-fat.

† Vasodilator function measured by flow-mediated dilatation, except López-Uriarte et al. $(2010)^{(106)}$, measured by Endo-PAT device.

$\ddagger$ Mixed nuts = walnuts, almonds and hazelnuts. 


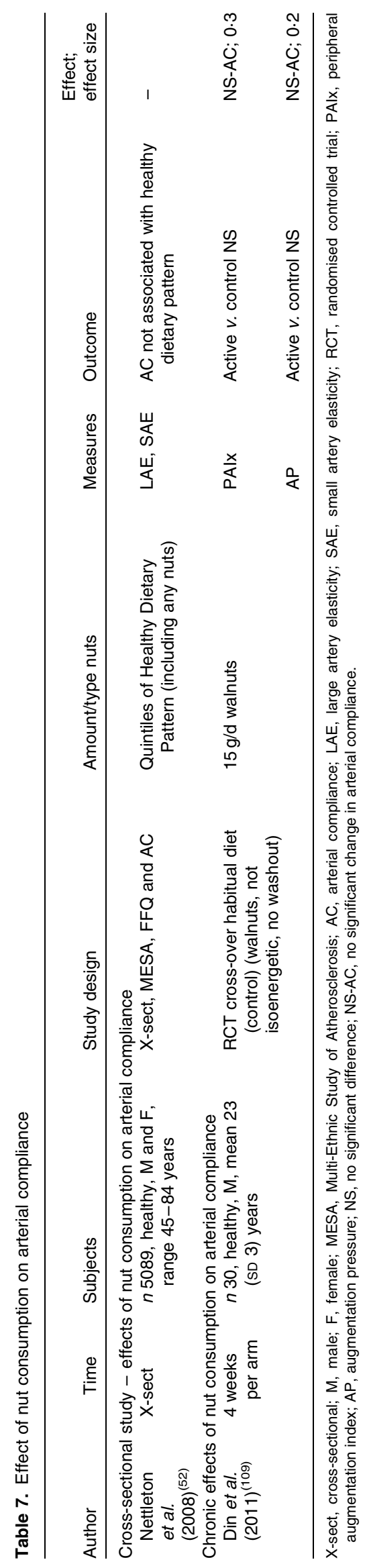

observational studies; hence intervention studies are important to isolate effects.

Significant reductions in blood pressure were observed in nine intervention studies ${ }^{(46,47,58,67,76,82-84)}$. Effect sizes could be calculated in seven of these and were small to large, ranging between $0 \cdot 2$ and $1 \cdot 1$. A substantial reduction in systolic blood pressure $(14 \mathrm{mmHg})$ was reported in participants who were overweight or obese and mildly hypertensive consuming a diet containing $84 \mathrm{~g}$ almonds/d for 24 weeks, compared with an isoenergetic high-carbohydrate $\operatorname{diet}^{(76)}$, with some participants reducing or eliminating the use of antihypertensive medications during the duration of the study. A weight reduction of $7 \%$ (BMI reduction of $2.5 \mathrm{~kg} / \mathrm{m}^{2}$ ) was also observed in the participants consuming nuts compared with the control, despite the two groups being prescribed isoenergetic diets which would have accounted for at least some of the reduction in blood pressure observed ${ }^{(76)}$. The PREDIMED Study tested the consumption of a Mediterranean diet which included $30 \mathrm{~g}$ mixed nuts/d compared with a Mediterranean diet devoid of nuts ${ }^{(58)}$. The study found a significant reduction in systolic and diastolic blood pressure of 7 and 3 $\mathrm{mmHg}$, respectively. This study used a large cohort of 772 participants; subgroups of this study with 49-106 participants also reported similar reductions in blood pressure $^{(67,84,85)}$. A larger cohort of the PREDIMED Trial found only a significant reduction in diastolic blood pressure ${ }^{(83)}$. The NORDIET included nuts as part of the intervention $\operatorname{diet}^{(46)}$, and reductions were demonstrated in systolic and diastolic blood pressure of $6 \mathrm{mmHg}$ (effect size 0.6) and $2 \mathrm{mmHg}$ (effect size 0.3), respectively. Almonds ( $23 \mathrm{~g} / \mathrm{d}$ ) consumed of as part of a portfolio diet with plant sterols and soya for 1 year demonstrated a reduction in systolic and diastolic blood pressure in a single-phase prospective study $^{(82)}$. However, as no control group was used, it is possible that the regular clinic visits in this study increased participant awareness of hypertension as a CVD risk factor and other behaviour change may have contributed to the reduction in blood pressure in addition to the almond intervention $^{(82)}$; without a control group this could not be determined. Consumption of a Mediterranean diet including 20-50g walnuts/d compared with a prudent diet demonstrated reductions in systolic blood pressure of $3 \mathrm{mmHg}$ (effect size 0.7 ) and in diastolic blood pressure of $2 \mathrm{mmHg}$ (effect size $0 \cdot 7)^{(47)}$.

The majority of the remaining studies demonstrated either small blood pressure reductions which did not reach significance or no change. A reduction in systolic and diastolic blood pressure was observed with consumption of $40 \mathrm{~g}$ hazelnuts/d for 4 weeks from baseline; however, this was not significantly different from the reduction observed with cocoa used as the control ${ }^{(86)}$. Inclusion of a control food that is not likely to change inflammation or endothelial function may have been a better choice to determine the effects attributable to hazelnuts ${ }^{(87)}$. An ad libitum diet with $56 \mathrm{~g}$ walnuts/d consumed 


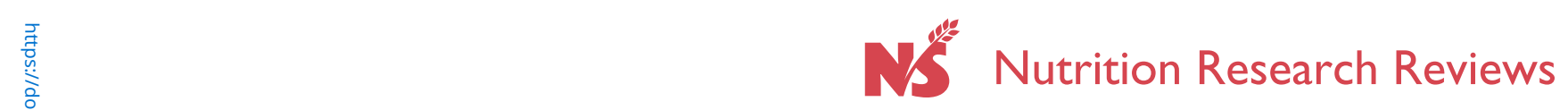

Table 8. Effects of nut consumption on cognitive function

\begin{tabular}{|c|c|c|c|c|c|c|}
\hline Author & Time & Subjects & Study design & Amount/type of nuts & Outcomes & $\begin{array}{l}\text { Effect; } \\
\text { effect size }\end{array}$ \\
\hline \multicolumn{7}{|c|}{ Observational studies: effects of nut consumption on cognitive function } \\
\hline $\begin{array}{l}\text { Valls-Pedret } \\
\text { et al. } \\
(2012)^{(158)}\end{array}$ & X-sect & $\begin{array}{l}n 447, \text { risk of CVD, } M \text { and } F \text {, } \\
\text { mean } 69 \text { (range 55-80) } \\
\text { years }\end{array}$ & $\begin{array}{l}\text { PREDIMED study, FFQ + } \\
\text { cognitive battery }\end{array}$ & $\begin{array}{l}5 \mathrm{~g} / \mathrm{d}(0-60 \mathrm{~g}) \text { all nuts, } 1 \mathrm{~g} / \mathrm{d} \\
\text { walnuts }(0-30 \mathrm{~g})\end{array}$ & $\begin{array}{l}\text { Walnuts (not other nuts) associated } \\
\text { with } \uparrow \text { working memory, } r 1.2 \\
(95 \% \mathrm{Cl} 0.06,2.32), \beta=0.15 \\
(P=0.04)\end{array}$ & + \\
\hline $\begin{array}{l}\text { Nooyens } \\
\text { et al. } \\
(2011)^{(110)}\end{array}$ & 5 years & $\begin{array}{l}n 2613, \text { general population, } \\
\text { range } 43-70 \text { years }\end{array}$ & $\begin{array}{l}\text { The Doetinchem Prospective } \\
\text { Cohort Study, FFQ and } \\
\text { cognitive battery }\end{array}$ & $\begin{array}{l}\text { Quintiles of any nut consump- } \\
\text { tion (amount not specified) }\end{array}$ & $\begin{array}{l}\text { (1) } \uparrow \text { Nut intake associated with } \uparrow \\
\text { cognitive function (memory, } \\
\text { speed, flexibility and global) } \\
(P \text {-trend }<0.01) \\
\text { (2) } \uparrow 5-8 \text { years cognitive function } \\
\text { in high } v \text {. low nut consumers } \\
\text { (3) No } \downarrow \text { cognitive decline in nut } \\
\text { consumers over } 5 \text { years }\end{array}$ & + \\
\hline $\begin{array}{l}\text { Nurk et al. } \\
(2010)^{(111)}\end{array}$ & $X$-sect & $\begin{array}{l}n 2031, M \text { and } F \text {, elderly, range } \\
70-74 \text { years }\end{array}$ & X-sect, FFQ + cognitive battery & $\begin{array}{l}\text { Mean intake of nut } \\
\text { consumers }=5 \mathrm{~g} / \mathrm{d}\end{array}$ & $\begin{array}{l}\text { Nut intake associated with } \uparrow \\
\text { executive function, semantic } \\
\text { memory, NS }\end{array}$ & NS-CP \\
\hline \multicolumn{7}{|c|}{ Chronic effects of nut consumption on cognitive function } \\
\hline \multirow[t]{2}{*}{$\begin{array}{l}\text { Pribis et al. } \\
(2012)^{(112)}\end{array}$} & 8 weeks & $\begin{array}{l}n 64, \mathrm{M} \text { and } \mathrm{F} \text {, students, mean } \\
21 \text { (SD 2) years }\end{array}$ & $\begin{array}{l}\text { RCT cross-over, banana bread } \\
\text { (control) with/without walnuts, } \\
6 \text { weeks washout }\end{array}$ & $60 \mathrm{~g} / \mathrm{d}$ walnuts & $\begin{array}{l}\text { Inferential verbal reasoning } \uparrow 11 \% \\
\quad(\mathrm{~d}=0.6 ; P=0.009)\end{array}$ & $+; 0.4$ \\
\hline & & & & & $\begin{array}{l}\text { Mood, non-verbal reasoning and } \\
\text { memory, NS }\end{array}$ & NS-CP \\
\hline
\end{tabular}

X-sect, cross-sectional; M, male; F, female; PREDIMED, PREvencion con Dleta MEDiterranea; $\uparrow$, increase; +, significant increase in cognitive performance; , decrease; NS, no significant change; NS-CP, no significant change in cognitive performance; RCT, randomised controlled trial. 


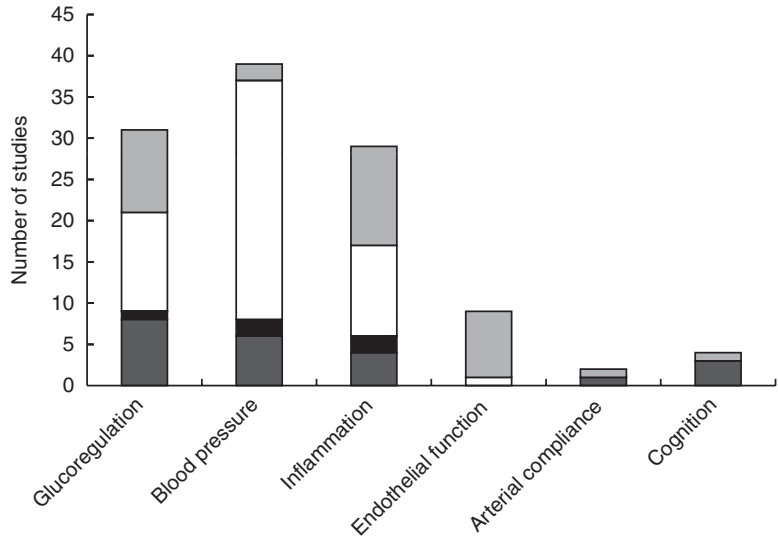

Fig. 3. Number of studies measuring effects of nut consumption on glucoregulation, blood pressure, inflammation, endothelial function, arterial compliance and cognition as epidemiological $(\square)$, uncontrolled $(\boldsymbol{\square})$ or randomised controlled trials with primary $(\square)$ or secondary $(\square)$ outcomes.

by participants with type 2 diabetes for 8 weeks showed an increase in systolic (effect size -0.8) and diastolic (effect size -0.7$)$ blood pressure. This unexpected result was from the only study that demonstrated a significant increase in blood pressure ${ }^{(72)}$. The authors were not able to determine a reason for this increase in blood pressure. However, other factors in the diet such as Na consumption may have contributed to the blood pressure elevation (despite being prescribed unsalted nuts); $\mathrm{Na}$ intake was not reported or controlled for in this study. Interventions using a portfolio diet ${ }^{(88,89)}$ NORDIET $^{(46)}$ or Mediterranean $\operatorname{diet}^{(47,48)}$ contained foods other than nuts which may also have been beneficial for improvements in blood pressure, making it difficult to tease out the effects of nuts alone. In contrast, Mediterranean diets in which mixed nuts ${ }^{(58,67)}$ replaced olive oil demonstrated improvements in blood pressure, indicating there may be some beneficial effect of nuts above that of other components of the Mediterranean diet. The largest effects of nuts on blood pressure were seen in participants with the metabolic syndrome or other risk factor for CVD, consuming 30-84 g of almonds, walnuts or mixed nuts/d for 4 weeks to 2 years. Significant reductions of 3-14 and 2-3 $\mathrm{mmHg}$ were observed in systolic and diastolic blood pressure, respectively $^{(46,47,58,67,76,84,85)}$. Only two of thirty-six studies measured resting blood pressure as a primary outcome, so the remaining studies may not have been powered to detect small changes. In eight of the nine studies demonstrating blood pressure reductions, nuts were consumed for extended periods of between 12 weeks to 2 years. Most studies demonstrated no beneficial effect on blood pressure when nuts were consumed for shorter periods (3-12 weeks). This suggests a benefit of nut consumption only after an extended period of time as indicated with observational studies where habitual nut consumption was associated with reduced blood pressure or reduced prevalence of hypertension.
Weighted mean changes in blood pressure were calculated for twenty-four of the thirty-six intervention studies; systolic and diastolic pressure were significantly reduced by $0.73(95 \% \mathrm{CI}-1.3,-0.2) \%$ and 0.75 (95\% CI -1.1 , $0 \cdot 4) \%$, respectively (see Table 9). Improvements in blood pressure control were observed particularly when nuts were consumed regularly for extended periods of time. Although the effect of nut consumption on blood pressure is small, this may still be clinically meaningful especially when used with other lifestyle measures.

\section{Effects of nut consumption on inflammatory markers}

Studies measuring the effect of nut consumption on inflammatory markers are found in Table 5. The most commonly measured inflammatory marker was CRP, reported in twenty-seven of the thirty-one studies. Other inflammatory markers measured included TNF- $\alpha$, interleukins (IL-1, IL-1 $\beta$, IL-6, IL-7 and IL-18) and cellular adhesion molecules (ICAM-1, VCAM-1 and E-selectin). We identified four crosssectional studies and twenty-seven intervention trials measuring inflammatory markers with nut consumption. Of the intervention studies, eleven compared nuts with a healthy diet (low-fat or Mediterranean diet), five with a Western, American or habitual diet, six studies compared nut consumption with another food product (meat, cocoa, lactose or olive oil), one study compared two types of nut and one study was a single intervention using pre- and post-measures with no control or comparator food. The range of nuts used included almonds, walnuts, mixed nuts, Brazil nuts, cashews, pistachios and hazelnuts. The amounts ranged from 10 to $103 \mathrm{~g}\left(\frac{1}{3}\right.$ ounce to 4 ounces) of nuts per d (approximately $5-25 \%$ of energy intake) for 4 weeks to 2 years. To date, only tree nuts have been tested for effects on inflammatory markers with chronic nut consumption.

In three of the four cross-sectional studies, nut consumption was associated with lower concentrations of the inflammatory markers CRP, IL-6 or ICAM. The MultiEthnic Study of Atherosclerosis (MESA) demonstrated an inverse association between frequency of consumption of nuts and seeds and serum CRP and IL-6 levels ${ }^{(90)}$. This association was moderately attenuated by additional adjustment for BMI. In two other studies, a Mediterranean diet pattern (PREDIMED study) or an American diet including nuts was inversely associated with anti-inflammatory markers ${ }^{(91)}$. Surprisingly, a large study (6309 women with diabetes), which categorised the largest nut consumption as $\geq 5$ serves per week ( 1 serve $=28 \mathrm{~g}$ nuts or $18 \mathrm{~g}$ peanut butter) showed no association with inflammatory markers $^{(92)}$.

A total of twelve intervention studies demonstrated significant reductions (5-75\%) in inflammatory markers with nut consumption with a variety of nuts. Consumption of 21-100 g of walnuts, almonds, hazelnuts, pistachios or mixed nuts per $\mathrm{d}$ for 4 weeks to 2 years in healthy 
or hypercholesterolaemic participants or those at high risk of CVD resulted in significant reductions of CRP $\quad(5-75 \%)^{(47,84,86,88,89,93-95)}$ or other inflammatory markers (ICAM, VCAM, E-selectin and interleukins) $(7-28 \%)^{(47,58,59,84,93,95-98)}$. A study incorporating walnuts $(37 \mathrm{~g} / \mathrm{d})$ plus walnut oil $(15 \mathrm{~g} / \mathrm{d})$ or walnuts and walnut oil plus flax seed as an additional source of ALA demonstrated anti-inflammatory effects compared with an American diet in hypercholesterolaemic individuals ${ }^{(93)}$. The vascular adhesion molecules ICAM-1, VCAM-1 as well as CRP were all reduced significantly, with a dose-response effect found for ALA in the diet with a $75 \%$ reduction in CRP. Participants who consumed $20-50 \mathrm{~g}$ walnuts/d for 2 years as part of a Mediterranean diet demonstrated a reduction in CRP (36\%) and interleukins IL-6, IL-7 and IL-18 (9-28\%) when compared with a prudent $\operatorname{diet}^{(47)}$. Mediterranean diets in which walnuts (about $65 \mathrm{~g} / \mathrm{d}$ ) or mixed nuts $(30 \mathrm{~g} / \mathrm{d})$ replaced olive oil demonstrated improvements in one or more of the inflammatory markers CRP, ICAM-1, VCAM-1 and IL-6 ${ }^{(58,84,98)}$. A reduction in CRP was demonstrated in four studies with a portfolio diet containing either almonds ${ }^{(89,94,99)}(14-30 \mathrm{~g} / \mathrm{d})$ or hazelnuts $(30 \mathrm{~g} / \mathrm{d})^{(86)}$ consumed for 4 weeks; one of these studies found an reduction equivalent to that observed with statin intake in the same individuals ${ }^{(89)}$. Beneficial improvements in inflammation observed in interventions which contained foods in addition to nuts (portfolio $\operatorname{diet}^{(88,89)}$ or Mediterranean $\operatorname{diet}^{(47,48)}$ ) may have been attributable to these other components. In contrast, Mediterranean diets in which walnuts ${ }^{(97)}$, mixed nuts ${ }^{(58,67)}$ or pistachios ${ }^{(59)}$ replaced olive oil demonstrated improvements in one or more of the inflammatory markers CRP, ICAM-1,VCAM-1 and IL- 6 , indicating that there may be some beneficial effect of nuts above that of other components of the Mediterranean diet. One study observed a reduction in CRP with a portfolio diet containing almonds only when participants with baseline CRP of $\leq 3.5 \mathrm{mg} / \mathrm{l}$ were excluded from analysis ${ }^{(94)}$. (CRP levels $\geq 3.5 \mathrm{mg} / 1$ reflect acute inflammation associated with infection or acute illness that would mask any potential effects of nuts on chronic inflammation. $)^{(100)}$ A $25 \%$ reduction in IL-6 was observed with a relatively large dose $(80-100 \mathrm{~g} / \mathrm{d})$ of pistachios consumed for 4 weeks ${ }^{(59)}$. Consumption of a high-almond diet $(68 \mathrm{~g} / \mathrm{d}$ per $2000 \mathrm{kcal}$ or $8368 \mathrm{~kJ})$ and a low-almond diet $(34 \mathrm{~g} / \mathrm{d}$ per $2000 \mathrm{kcal}$ or $8368 \mathrm{~kJ})$ for 4 weeks significantly decreased CRP compared with an isoenergetic control diet in healthy men and women ${ }^{(95)}$; E-selectin (a marker of endothelial inflammation) was significantly lower in the higher-almond group than control. No dose-response relationship was observed with either inflammatory marker in this study. In participants at risk of CVD, statistically significant reductions of the cellular adhesion molecules ICAM-1 (effect size 0.3) and VCAM-1 (effect size 0.4) were demonstrated with relatively low doses $(21 \mathrm{~g} / \mathrm{d})$ of walnuts added to a meat product compared with the meat product without walnuts. 
Despite little evidence for the magnitude of nut dose influencing inflammation, it is possible that there is a minimum dose required since no studies using $<30 \mathrm{~g} / \mathrm{d}$ demonstrated benefits.

In fifteen studies no significant changes in inflammatory markers were demonstrated, although most of these demonstrated small reductions. A recent three-arm study compared fatty fish $v$. walnuts $v$. a fish-/nut-free diet (control). No significant changes were found between the walnut and the control diets but E-selectin was reduced with the walnut intervention compared with the fish intervention $^{(96)}$. Consumption of $26 \mathrm{~g}$ almonds/d or $36 \mathrm{~g}$ walnuts/d for 6 weeks led to a $19 \%$ reduction in IL-6 with the almonds and $20 \%$ reduction in TNF- $\alpha$ with the walnuts compared with baseline, but this did not reach significance. In addition, two studies with obese individuals demonstrated small but non-significant improvements in $\mathrm{CRP}^{(101)}$ and IL-6 ${ }^{(60)}$ with Brazil nut and mixed nut consumption, respectively. Suggested reasons for small but non-significant reductions in inflammatory markers were recruitment of healthy individuals who may only demonstrate limited improvements and diurnal effects of IL-6 that are more difficult to detect than other markers. One study with obese individuals demonstrated small but non-significant improvements in $\mathrm{CRP}^{(101)}$. Increased central adiposity and body weight are associated with increased CRP levels and adipose pro-inflammatory cytokines including IL- $6^{(102)}$. It is possible that these individuals may not demonstrate improvements in inflammatory markers with a dietary intervention without weight loss.

The calculated weighted mean changes for all studies where data revealed reductions in ICAM-1, VCAM-1 and CRP were 8.6 (95\% CI $-20 \cdot 5,3 \cdot 3) \%, 5 \cdot 8$ (95\% CI $-14 \cdot 1$, $2 \cdot 5) \%$ and $12(95 \% \mathrm{CI}-23 \cdot 6,-0 \cdot 3) \%$, respectively (see Table 9). In summary, nut consumption has the potential to improve inflammatory markers, particularly with doses of $30 \mathrm{~g}$ or greater. This is in line with a health claim for nuts first established by the US Food and Drug Administration (FDA) in 2003; scientific evidence suggests that eating $42 \mathrm{~g}$ ( 1.5 ounces) of most nuts per $\mathrm{d}$ (as part of an overall healthy diet) may be able to reduce the risk of heart disease ${ }^{(103)}$

\section{Effects of nut consumption on endothelial vasodilator function}

Studies measuring the effect of nut consumption on endothelial vasodilator function are found in Table 6. In nine intervention studies the effect of nut consumption on endothelial vasodilator function (using either flowmediated dilatation or Endo-PAT device) was measured, with the dose of nuts ranging from 10 to $100 \mathrm{~g} / \mathrm{d}$ for periods ranging from 4 to 12 weeks. Of the nine studies, five demonstrated a significant effect.

Endothelial function was significantly improved (24-64\%) in healthy and hypercholesterolaemic participants who consumed $37-100 \mathrm{~g}$ of walnuts or pistachios per $\mathrm{d}$ for 4-8 weeks ${ }^{(59,72,98,104)}$. A Mediterranean diet supplemented with $65 \mathrm{~g}$ walnuts/d substituted for olive oil significantly improved vasodilation by $64 \%$ in hypercholesterolaemic adults $^{(98)}$. This study also demonstrated an inverse association between vasodilation and cholesterol:HDL ratio, suggesting that the effect of walnuts may have been mediated in part through an improved lipid profile ${ }^{(98)}$. It is well established that hypercholesterolaemia impairs endothelial function, which can be reversed by aggressive cholesterol lowering ${ }^{(105)}$. However, this study only demonstrated moderate cholesterol lowering, indicating that other mechanisms may also play a role. Investigators suggested that phenolic compounds in walnuts may have counteracted the pro-oxidant effects of PUFA on LDL. Mediterranean diets supplemented with $20-50 \mathrm{~g}$ walnuts/d $\mathrm{d}^{(47)}$ and 80-100 g pistachios/ $\mathrm{d}^{(59)}$ improved endothelial function by 21 and $24 \%$, respectively. Also observed with pistachio consumption was an improvement in glucose levels, lipid parameters, oxidative status and some indices of inflammation that may underlie the improved endothelial function ${ }^{(59)}$. A diet with ad libitum consumption $(56 \mathrm{~g} / \mathrm{d})$ of walnuts improved endothelial function by $45 \%$ (effect size 0.6) in participants with type 2 diabetes $^{(72)}$. Consumption of walnuts and walnut oil supplemented with flax seed (to boost the ALA content of the diet) increased endothelial function by $34 \%$, but no change was observed with the walnut diet alone ${ }^{(104)}$. Of the five studies using higher doses $(56-100 \mathrm{~g} / \mathrm{d})$ of nuts, four demonstrated benefits on endothelial function, indicating that higher doses may be required to elicit benefits ${ }^{(59,72,98,104)}$.

Of the studies, four did not show significant effects on flow-mediated dilatation. A hazelnut-enriched diet consumed by healthy men improved lipid parameters. In spite of this, endothelial functional improvement did not reach statistical significance ${ }^{(74)}$. A quantity of $10-30 \mathrm{~g}$ nuts per d consumed by participants with either CVD risk or the metabolic syndrome also demonstrated no benefits on endothelial function ${ }^{(48,106)}$. Consumption of two doses (10 and $20 \%$ of energy) of pistachios did not lead to a reduction in endothelial function ${ }^{(107)}$ despite relatively high doses of up to $126 \mathrm{~g} / \mathrm{d}$ consumed for 4 weeks. The authors suggested that pistachios used were roasted which may have reduced polyphenol activity unlike walnuts used in other studies, which were not roasted before consumption.

Calculations of the weighted mean changes from nine of the ten studies indicated a $19 \cdot 7$ (95\% CI 4.3, 35.0)\% relative increase in vasodilatation with nut consumption (Table 9). The effects of nuts on endothelial function demonstrate potential benefits, particularly walnuts. However, limited studies have been conducted with other types of nuts that may also demonstrate benefits. Endothelial dysfunction is often detected before increased blood pressure is observed and may be a more sensitive indicator than arterial compliance of early decline in 
vascular health; hence it may be a better target than blood pressure control or arterial compliance ${ }^{(108)}$.

\section{Effects of nut consumption on arterial compliance}

Studies measuring the effect of nut consumption on arterial compliance are found in Table 7. These include one crosssectional study and one intervention study. A dose of $15 \mathrm{~g}$ walnuts/d consumed for 4 weeks demonstrated no effect on arterial stiffness ${ }^{(109)}$. Whilst this dose is small, investigators chose a realistic amount likely to be consumed in free-living individuals for an extended period of time rather than higher doses used in other nut intervention studies. The cross-sectional study measured arterial compliance and compared quintiles of a healthy dietary pattern including nuts ${ }^{(52)}$. No association was found between a healthier diet pattern with an undetermined quantity of nuts and measures of arterial compliance. Few studies have investigated the effects of nuts on arterial compliance; therefore more studies in this area are warranted.

\section{Effects of nut consumption on cognitive performance}

There is little known of the impact of nut consumption on cognitive function. Studies measuring the effect of nut consumption on cognitive performance are found in Table 8. A 5-year prospective cohort study demonstrated a positive association between nut consumption and cognitive performance, equivalent to a substantial age reduction effect of 5-8 years in the highest-nut consumers (amount of nuts not specified) ${ }^{(110)}$. In addition, cognitive performance did not decline over the 5-year period in the highest-nut consumers. In a cross-sectional study (PREDIMED) an association was found between walnut consumption (but not other nuts) and improvements in performance on tests of working memory (see Table 8). In older adults nut consumption was associated with improved but nonsignificant scores for executive function in a cross-sectional study ${ }^{(111)}$, with a low mean intake of nuts of $5 \mathrm{~g} / \mathrm{d}$. Only one intervention study in human subjects has been performed; this was conducted with students consuming $60 \mathrm{~g}$ ground walnuts/d for 8 weeks ${ }^{(112)}$. The study demonstrated a medium effect size (0.4) for improvement in inferential reasoning; however, other cognitive tests demonstrated no change. Despite the lack of intervention trials, observational studies indicate that long-term consumption of even small amounts of nuts may elicit benefits for cognitive function and reduction in cognitive decline. More evidence is needed from controlled intervention studies before a conclusive benefit can be determined.

\section{Proposed mechanisms}

Several nutrients in nuts may be responsible for observed improvements in cardiometabolic and cognitive measures. Tree and ground nuts have similar nutrient profiles, with some variations in micro- and macronutrients. From the studies reviewed (with the exception of walnuts, which have been more extensively researched than other nuts), it is not possible to determine differences in efficacy between different types of tree and ground nuts. Walnuts differ from other nuts in their greater antioxidant capacity, polyphenol and ALA content (see Table 1). ALA found in walnuts is associated with improved endothelial function $^{(31)}$, inflammation ${ }^{(113)}$ and neuroprotection in animal models ${ }^{(114)}$ and is hypothesised to maintain cognitive function in older adults ${ }^{(115)}$. Other unsaturated fatty acids in nuts may be beneficial for insulin sensitivity ${ }^{(116)}$ and evidence suggests that higher intakes are associated with a lower risk of type 2 diabetes ${ }^{(117)}$, whereas higher intakes of SFA adversely affect glucose metabolism and insulin resistance ${ }^{(118-120)}$. There is also recent evidence to indicate that MUFA may contribute to improvements in arterial stiffness as well as endothelial function and inflammation $^{(121-124)}$. Consumption of a Mediterranean diet that is also high in MUFA has been shown to reduce VCAM-1 and E-selectin gene expression by almost half. Animal and human studies have demonstrated that inflammation can be modified by the intake of L-arginine ${ }^{(125)}$. Individuals with hypercholesterolaemia have impaired synthesis of $\mathrm{NO}$; supplementation of $7 \mathrm{~g} \mathrm{~L}$-arginine/d in this population group has demonstrated benefits ${ }^{(126)}$, increasing endothelial-dependent dilatation by almost $3 \cdot 5$-fold. Nuts contain approximately $2-3 \mathrm{~g}$ arginine $/ 100 \mathrm{~g}$; hence doses of $30 \mathrm{~g} / \mathrm{d}$ or more used in most studies could partly account for the improvement in endothelial function observed. Nuts also contain fibre and, when consumed with their skin intact, contain a significant amount of polyphenols ${ }^{(35,127)}$, which have previously been shown to target endothelial cells resulting in improved vascular function $^{(42,128,129)}$. Fibre intake can also increase insulin sensitivity $^{(130,131)}$. Vitamin $\mathrm{E}$ found in nuts may have a role in modifying some of the inflammatory mediators and may be beneficial for cognitive performance ${ }^{(38,40)} \cdot \gamma$-Tocopherol is a powerful antioxidant abundant in walnuts, Brazil nuts and pistachios; however, its effect on markers of cardiovascular risk including endothelial function and inflammation has not yet been determined. Nuts are naturally rich in $\mathrm{K}$ and $\mathrm{Mg}$, which may facilitate blood pressure reductions unless consumed in the salted form ${ }^{(132)}$. In addition, $\mathrm{Mg}$, which has been inversely related to serum CRP levels, has the potential to improve inflammation in individuals with low Mg status ${ }^{(133)}$ and $\mathrm{Mg}$ intake is inversely associated with a reduced risk of type 2 diabetes ${ }^{(134)}$.

There is emerging evidence that frequent nut consumption beneficially affects cardiovascular risk beyond cholesterol lowering. Key mechanisms include anti-inflammatory, antioxidant and endothelial function, reduction in body fat and improvement in glucose metabolism, which play a central role in the development of atherosclerosis ${ }^{(135,136)}$. Endothelial function is essential for cerebral vascular function to provide adequate cerebral blood flow to deliver 
nutrients (primarily glucose and oxygen) to the brain. It has been hypothesised that by improving blood-flow regulation in the brain, cognitive performance is also improved $^{(6,7)}$. Nutritional interventions that have demonstrated improvements in cerebral blood flow include $n-3$ fatty acids in fish oil ${ }^{(137)}$, polyphenols in $\operatorname{cocoa}^{(138)}$ and wild green oats ${ }^{(139)}$. Anti-inflammatory medications offer some protection from Alzheimer's disease, which is consistent with the hypothesis that damage to brain cells is part of an overall inflammatory reaction. If inflammation is the key, then nuts which contain anti-inflammatory nutrients, such as polyphenols, vitamin $\mathrm{E}$ and $n-3$ fatty acids may prove to be important to reduce damage to the brain.

\section{Conclusions}

The results summarised in the present study provide evidence that regular nut consumption may have a protective effect on both vascular health and cognition. These benefits were evident in trials with doses of higher intakes $(>30 \mathrm{~g} / \mathrm{d})$ for extended periods (several weeks or longer). These findings further support the use of nuts to reduce cardiometabolic dysfunction and highlight their potential to maintain or restore endothelial function. This in turn could improve cerebral blood flow and hence cognitive performance as illustrated in Fig. 1. No published studies to date have measured the effect of nut consumption on cerebral blood flow and few studies have measured the impact of nuts on arterial compliance and cognitive performance. Whilst intervention studies have investigated the impact of nuts on endothelial function, only one study has taken the next step and considered whether nuts may have beneficial effects on cognitive performance. Further clinical studies are warranted to determine the type and dose of nut and duration of consumption and which populations may benefit.

\section{Acknowledgements}

J. A. B. is funded by a scholarship from the Australian Research Council linkage grant in partnership with the Peanut Company of Australia (no. LP100200597).

There are no declarations of conflict of interest.

\section{References}

1. Yazdanyar A \& Newman AB (2009) The burden of cardiovascular disease in the elderly: morbidity, mortality, and costs. Clin Geriatr Med 25, 563-577.

2. Drag LL \& Bieliauskas LA (2010) Contemporary review 2009: cognitive aging. J Geriatr Psychiatry Neurol 23, 75-93.

3. Brookmeyer R, Johnson E, Ziegler-Graham K, et al. (2007) Forecasting the global burden of Alzheimer's disease. Alzheimers Dement 3, 186-191.
4. Monsuez J-J, Gesquière-Dando A \& Rivera S (2011) Cardiovascular prevention of cognitive decline. Cardiol Res Pract 2011, 250970-250977.

5. Deary IJ, Corley J, Gow AJ, et al. (2009) Age-associated cognitive decline. Br Med Bull 92, 135-152.

6. Sinn N \& Howe PRC (2008) Mental health benefits of omega-3 fatty acids may be mediated by improvements in cerebral vascular function. Biosci Hypotheses 1, 103-108.

7. Krestin GP, van der Lugt A, Poels MMF, et al. (2008) Total cerebral blood flow in relation to cognitive function: the Rotterdam Scan Study. J Cereb Blood Flow Metab 28, $1652-1655$.

8. Silvestrini M, Pasqualetti P, Baruffaldi R, et al. (2006) Cerebrovascular reactivity and cognitive decline in patients with Alzheimer disease. Stroke 37, 1010-1015.

9. Iadecola C \& Davisson RL (2008) Hypertension and cerebrovascular dysfunction. Cell Metab 7, 476-484.

10. Arfanakis K, Fleischman DA, Grisot G, et al. (2013) Systemic inflammation in non-demented elderly human subjects: brain microstructure and cognition. PLOS ONE 8, e73107.

11. Meigs JB, Hu FB, Rifai N, et al. (2004) Biomarkers of endothelial dysfunction and risk of type 2 diabetes mellitus. $J \mathrm{Am}$ Med Assoc 291, 1978-1986.

12. Bomboi G, Castello L, Cosentino F, et al. (2010) Alzheimer's disease and endothelial dysfunction. Neurol Sci 31, 1-8.

13. Singhal A (2005) Endothelial dysfunction: role in obesityrelated disorders and the early origins of CVD. Proc Nutr Soc 64, 15.

14. Lundberg JO \& Gladwin MT (2008) \& Weitzberg E The nitrate-nitrite-nitric oxide pathway in physiology and therapeutics. Nat Rev Drug Discov 7, 156-167.

15. Rana JS, Nieuwdorp M, Jukema JW, et al. (2007) Cardiovascular metabolic syndrome - an interplay of obesity, inflammation, diabetes and coronary heart disease. Diabetes Obes Metab 9, 218-232.

16. Vita JA (2011) Endothelial function. Circulation $\mathbf{1 2 4}$, E906-E912.

17. Benton D (2001) The impact of the supply of glucose to the brain on mood and memory. Nutr Rev 59, S20-S21.

18. Bourre JM (2006) Effects of nutrients (in food) on the structure and function of the nervous system: update on dietary requirements for brain. Part 1: micronutrients. J Nutr Health Aging 10, 377-385.

19. Benarroch EE (2012) Blood-brain barrier: recent developments and clinical correlations. Neurology 78, 1268-1276.

20. Coates AM \& Howe PRC (2007) Edible nuts and metabolic health. Curr Opin Lipidol 18, 25-30.

21. Casas-Agustench P, Bulló M \& Salas-Salvadó J (2010) Nuts, inflammation and insulin resistance. Asia Pac J Clin Nutr 19, 124-130.

22. Bes-Rastrollo M, Sabaté J, Gómez-Gracia E, et al. (2007) Nut consumption and weight gain in a Mediterranean cohort: the SUN Study. Obesity 15, 107-116.

23. Kris-Etherton PM \& Griel AE (2006) Tree nuts and the lipid profile: a review of clinical studies. Br J Nutr 96, S68-S78.

24. Kelly JH \& Sabaté J (2006) Nuts and coronary heart disease: an epidemiological perspective. Br J Nutr 96, S61-S67.

25. Banel D \& Hu F (2009) Effects of walnut consumption on blood lipids and other cardiovascular risk factors: a meta-analysis and systematic review. Am J Clin Nutr 90, 56-63.

26. Sabate J, Oda K \& Ros E (2010) Nut consumption and blood lipid levels. A pooled analysis of 25 intervention trials. Arch Intern Med 170, 821-827.

27. Kendall CWC, Josse AR, Esfahani A, et al. (2010) Nuts, metabolic syndrome and diabetes. Br J Nutr 104, 465-473. 
28. Casas-Agustench P, López-Uriarte P, Ros E, et al. (2011) Nuts, hypertension and endothelial function. Nutr Metab Cardiovasc Dis 21, Suppl. 1, S21-S33.

29. Gopinath B, Buyken AE, Flood VM, et al. (2011) Consumption of polyunsaturated fatty acids, fish, and nuts and risk of inflammatory disease mortality. Am J Clin Nutr 93, 1073-1079.

30. Salas-Salvadó J, Bulló M, Babio N, et al. (2011) Reduction in the incidence of type 2 diabetes with the Mediterranean diet: results of the PREDIMED-Reus nutrition intervention randomized trial. Diabetes Care 34, 14-19.

31. Mozaffarian D (2005) Does $\alpha$-linolenic acid intake reduce the risk of coronary heart disease? A review of the evidence. Alternat Ther Health Med 11, 24-30.

32. Paschos GK, Magkos F, Panagiotakos DB, et al. (2007) Dietary supplementation with flaxseed oil lowers blood pressure in dyslipidaemic patients. Eur J Clin Nutr 61, 1201-1206.

33. Sala-Vila A \& Ros E (2011) Mounting evidence that increased consumption of $\alpha$-linolenic acid, the vegetable $n$-3 fatty acid, may benefit cardiovascular health. Clin Lipidol 6, 365-369.

34. Muramatsu T, Yatsuya H, Toyoshima H, et al. (2010) Higher dietary intake of $\alpha$-linolenic acid is associated with lower insulin resistance in middle-aged Japanese. Prev Med $\mathbf{5 0}$, 272-276.

35. Bolling BW, Chen CYO, McKay DL, et al. (2011) Tree nut phytochemicals: composition, antioxidant capacity, bioactivity, impact factors. A systematic review of almonds, Brazils, cashews, hazelnuts, macadamias, pecans, pine nuts, pistachios and walnuts. Nutr Res Rev 24, 244-275.

36. Lecour S \& Lamont KT (2011) Natural polyphenols and cardioprotection. Mini Rev Med Chem 11, 1191-1199.

37. Biesalski HK (2007) Polyphenols and inflammation: basic interactions. Curr Opin Clin Nutr Metab Care 10, 724-728.

38. Singh U, Devaraj S \& Jialal I (2005) Vitamin E, oxidative stress, and inflammation. Annu Rev Nutr 25, 151-174.

39. Collie A \& Morley G (2007) Do polyphenols affect human cognitive function? Curr Top Neutraceut Res 5, 145-148.

40. Joshi YB \& Praticò D (2012) Vitamin E in aging, dementia and Alzheimer's disease. BioFactors 38, 90-97.

41. Sánchez-Muniz FJ (2012) Dietary fibre and cardiovascular health. Nutr Hosp 27, 31-45.

42. Brock DW, Davis CK, Irving BA, et al. (2006) A high-carbohydrate, high-fiber meal improves endothelial function in adults with the metabolic syndrome. Diabetes Care 29, $2313-2315$.

43. King DE (2005) Dietary fiber, inflammation, and cardiovascular disease. Mol Nutr Food Res 49, 594-600.

44. Lekakis JP, Papaioannou TG, Stamatelopoulos SF, et al. (2002) Oral l-arginine improves endothelial dysfunction in patients with essential hypertension. Int J Cardiol 86, 317-323.

45. National Health and Medical Research Council (2000) How to Use the Evidence: Assessment and Application of Scientific Evidence. Canberra: National Health and Medical Research Council

46. Adamsson V, Reumark A, Fredriksson IB, et al. (2011) Effects of a healthy Nordic diet on cardiovascular risk factors in hypercholesterolaemic subjects: a randomized controlled trial (NORDIET). J Intern Med 269, 150-159.

47. Esposito K, Giugliano G, Giugliano F, et al. (2004) Effect of a Mediterranean-style diet on endothelial dysfunction and markers of vascular inflammation in the metabolic syndrome: a randomized trial. JAMA 292, 1440-1446.

48. Thomazella MCD, Góes MFS, Andrade CR, et al. (2011) Effects of high adherence to Mediterranean or low-fat diets in medicated secondary prevention patients. Am J Cardiol 108, 1523-1529.

49. O'Neil CE, Keast DR, Nicklas TA, et al. (2011) Nut consumption is associated with decreased health risk factors for cardiovascular disease and metabolic syndrome in U.S. adults: NHANES 1999-2004. I Am Coll Nutr 30, 502-510.

50. Alvarez León EE, Henríquez P \& Serra-Majem L (2006) Mediterranean diet and metabolic syndrome: a cross-sectional study in the Canary Islands. Public Health Nutr 9, 1089-1098

51. Jiang R, Liu S, Manson JE, et al. (2002) Nut and peanut butter consumption and risk of type 2 diabetes in women. JAMA 288, 2554-2560.

52. Nettleton JA, Schulze MB, Jiang R, et al. (2008) A prioridefined dietary patterns and markers of cardiovascular disease risk in the Multi-Ethnic Study of Atherosclerosis (MESA). Am J Clin Nutr 88, 185-194.

53. Martínez-González MÁ, de la Fuente-Arrillaga C, NunezCordoba JM, et al. (2008) Adherence to Mediterranean diet and risk of developing diabetes: prospective cohort study. BMJ 336, 1348-1351.

54. Pan A, Sun Q, Manson JE, et al. (2013) Walnut consumption is associated with lower risk of type 2 diabetes in women. J Nutr 143, 512-518.

55. Villegas R, Gao Y-T, Yang G, et al. (2008) Legume and soy food intake and the incidence of type 2 diabetes in the Shanghai Women's Health Study. Am J Clin Nutr 87, 162-167.

56. Biesalski HK (2004) Diabetes preventive components in the Mediterranean diet. Eur J Nutr 43, Suppl. 1, 26-30.

57. Meyer KA, Kushi LH, Jacobs DR Jr, et al. (2001) Dietary fat and incidence of type 2 diabetes in older Iowa women. Diabetes Care 24, 1528-1535.

58. Estruch R, Martínez-González MA, Corella D, et al. (2006) Effects of a Mediterranean-style diet on cardiovascular risk factors: a randomized trial. Ann Intern Med 145, 1-11.

59. Sari I, Baltaci Y, Bagci C, et al. (2010) Effect of pistachio diet on lipid parameters, endothelial function, inflammation, and oxidative status: a prospective study. Nutrition $\mathbf{2 6}$, 399-404.

60. Casas-Agustench P, López-Uriarte P, Bulló M, et al. (2011) Effects of one serving of mixed nuts on serum lipids, insulin resistance and inflammatory markers in patients with the metabolic syndrome. Nutr Metab Cardiovasc Dis 21, 126-135.

61. Wien M, Bleich D, Raghuwanshi M, et al. (2010) Almond consumption and cardiovascular risk factors in adults with prediabetes. J Am Coll Nutr 29, 189-197.

62. Kalgaonkar S, Almario RU, Gurusinghe D, et al. (2011) Differential effects of walnuts vs almonds on improving metabolic and endocrine parameters in PCOS. Eur J Clin Nutr 65, 386-393.

63. Zaveri S \& Drummond S (2009) The effect of including a conventional snack (cereal bar) and a nonconventional snack (almonds) on hunger, eating frequency, dietary intake and body weight. J Hum Nutr Diet 22, 461-468.

64. Cohen AE \& Johnston CS (2011) Almond ingestion at mealtime reduces postprandial glycemia and chronic ingestion reduces hemoglobin A1c in individuals with well-controlled type 2 diabetes mellitus. Metabolism 60, 1312-1317.

65. Lovejoy JC, Most MM, Lefevre M, et al. (2002) Effect of diets enriched in almonds on insulin action and serum lipids in adults with normal glucose tolerance or type 2 diabetes Am J Clin Nutr 76, 1000-1006.

66. Jenkins DJA, Srichaikul K, Banach MS, et al. (2011) Nuts as a replacement for carbohydrates in the diabetic diet. Diabetes Care 34, 1706-1711. 
67. Llorente-Cortés V, Estruch R, Mena MP, et al. (2010) Effect of Mediterranean diet on the expression of pro-atherogenic genes in a population at high cardiovascular risk. Atherosclerosis 208, 442-450.

68. Wu H, Xiafei C, Lixin T, et al. (2010) Lifestyle counseling and supplementation with flaxseed or walnuts influence the management of metabolic syndrome. J Nutr $\mathbf{1 4 0}$, 1937-1942.

69. Tapsell LC, Gillen LJ, Patch CS, et al. (2004) Including walnuts in a low-fat/modified-fat diet improves HDL cholesterol-to-total cholesterol ratios in patients with type 2 diabetes. Diabetes Care 27, 2777-2783.

70. Tapsell LC, Batterham MJ, Teuss G, et al. (2009) Long-term effects of increased dietary polyunsaturated fat from walnuts on metabolic parameters in type II diabetes. Eur J Clin Nutr 63, 1008-1015.

71. Mukuddem-Petersen J, Stonehouse Oosthuizen W, Jerling JC, et al. (2007) Effects of a high walnut and high cashew nut diet on selected markers of the metabolic syndrome: a controlled feeding trial. Br J Nutr 97, 1144-1153.

72. Ma Y, Njike VY, Millet J, et al. (2010) Effects of walnut consumption on endothelial function in type 2 diabetic subjects: a randomized controlled crossover trial. Diabetes Care 33, 227-232.

73. Kasim-Karakas SE, Almario RU, Gregory L, et al. (2004) Metabolic and endocrine effects of a polyunsaturated fatty acid-rich diet in polycystic ovary syndrome. $J$ Clin Endocrinol Metab 89, 615-620.

74. Mercanligil SM, Arslan P, Alasalvar C, et al. (2007) Effects of hazelnut-enriched diet on plasma cholesterol and lipoprotein profiles in hypercholesterolemic adult men. Eur J Clin Nutr 61, 212-220.

75. Li Z, Song R, Nguyen C, et al. (2010) Pistachio nuts reduce triglycerides and body weight by comparison to refined carbohydrate snack in obese subjects on a 12-week weight loss program. J Am Coll Nutr 29, 198-203.

76. Wien M, Kandeel F, Sabate J, et al. (2003) Effects of almonds $v s$. complex carbohydrates on metabolic syndrome and anthropometric parameters during weight reduction. Diabetes 52, A395-A399.

77. Djoussé L, Gaziano JM, Kase CS, et al. (2010) Nut consumption and risk of stroke in US male physicians. Clin Nutr $\mathbf{2 8}$, $10-14$.

78. Steffen LM, Kroenke CH, Yu X, et al. (2005) Associations of plant food, dairy product, and meat intakes with 15 -y incidence of elevated blood pressure in young black and white adults: the Coronary Artery Risk Development in Young Adults (CARDIA) Study. Am J Clin Nutr 82, 1169-1177.

79. Weng L-C, Steffen LM, Szklo M, et al. (2013) A diet pattern with more dairy and nuts, but less meat is related to lower risk of developing hypertension in middle-aged adults: the Atherosclerosis Risk in Communities (ARIC) Study. Nutrients 5, 1719-1733.

80. Martínez-Lapiscina EH, Pimenta AM, Beunza JJ, et al. (2010) Nut consumption and incidence of hypertension: the SUN prospective cohort. Nutr Metab Cardiovasc Dis 20 , 359-365.

81. Ibarrola-Jurado N, Bulló M, Guasch-Ferré M, et al. (2013) Cross-sectional assessment of nut consumption and obesity, metabolic syndrome and other cardiometabolic risk factors: the PREDIMED study. PLOS ONE 8, e57367.

82. Jenkins DJA, Vidgen E, Trautwein EA, et al. (2008) Longterm effects of a plant-based dietary portfolio of cholesterol-lowering foods on blood pressure. Eur J Clin Nutr 62, 781-788.

83. Toledo E, Hu FB, Estruch R, et al. (2013) Effect of the Mediterranean diet on blood pressure in the PREDIMED trial results from a randomized controlled trial. Complement Altern Med 11, 207-216.

84. Mena M-P, Casas R, Lamuela-Raventós RM, et al. (2009) Inhibition of circulating immune cell activation: a molecular antiinflammatory effect of the Mediterranean diet. Am J Clin Nutr 89, 248-256.

85. Fito M, Marrugat J, Garcia-Arellano A, et al. (2007) Effect of a traditional Mediterranean diet on lipoprotein oxidation: a randomized controlled trial. Arch Intern Med 167 , 1195-1203.

86. Solà R, Valls RM, Godàs G, et al. (2012) Cocoa, hazelnuts, sterols and soluble fiber cream reduces lipids and inflammation biomarkers in hypertensive patients: a randomized controlled trial. PLOS ONE 7, e31103.

87. Davison K, Berry NM, Misan G, et al. (2010) Dose-related effects of flavanol-rich cocoa on blood pressure. $J$ Hum Hypertens 24, 568-576.

88. Jenkins DJA, Josse RG, Leiter LA, et al. (2002) A dietary portfolio approach to cholesterol reduction: combined effects of plant sterols, vegetable proteins, and viscous fibers in hypercholesterolemia. Metabolism 51, 1596-1604.

89. Jenkins DJA, Lapsley KG, Trautwein EA, et al. (2003) Effects of a dietary portfolio of cholesterol-lowering foods vs lovastatin on serum lipids and C-reactive protein. J Am Med Assoc 290, $502-510$.

90. Jiang R, Jacobs DR Jr, Mayer-Davis E, et al. (2006) Nut and seed consumption and inflammatory markers in the multiethnic study of atherosclerosis. Am J Epidemiol 163, 222-231.

91. Salas-Salvadó J, Casas-Agustench P, Murphy MM, et al. (2008) The effect of nuts on inflammation. Asia Pac J Clin Nutr 19, Suppl. 1, 124-130.

92. Li TY, Brennan AM, Wedick NM, et al. (2009) Regular consumption of nuts is associated with a lower risk of cardiovascular disease in women with type 2 diabetes. J Nutr 139, 1333-1338.

93. Zhao G, Etherton TD, Martin KR, et al. (2004) Dietary $\alpha$-linolenic acid reduces inflammatory and lipid cardiovascular risk factors in hypercholesterolemic men and women. J Nutr 134, 2991-2997.

94. Jenkins DJA, Li TJ, Josse RG, et al. (2005) Direct comparison of dietary portfolio vs statin on C-reactive protein. Eur J Clin Nutr 59, 851-860.

95. Rajaram S \& Connell KM \& Sabaté J (2010) Effect of almondenriched high-monounsaturated fat diet on selected markers of inflammation: a randomised, controlled, crossover study. Br J Nutr 103, 907-912.

96. Chiang Y-L, Haddad E, Rajaram S, et al. (2012) The effect of dietary walnuts compared to fatty fish on eicosanoids, cytokines, soluble endothelial adhesion molecules and lymphocyte subsets: a randomized, controlled crossover trial. Prostaglandins Leukot Essent Fatty Acids 87, 111-117.

97. Canales A, Sánchez-Muniz FJ, Bastida S, et al. (2011) Effect of walnut-enriched meat on the relationship between VCAM, ICAM, and LTB4 levels and PON-1 activity in ApoA4 360 and PON-1 allele carriers at increased cardiovascular risk. Eur J Clin Nutr 65, 703-710.

98. Ros E, Núñez I, Pérez-Heras A, et al. (2004) A walnut diet improves endothelial function in hypercholesterolemic subjects: a randomized crossover trial. Circulation 109, 1609-1614.

99. Jenkins DJA, Kendall CWC, Marchie A, et al. (2002) Dose response of almonds on coronary heart disease risk factors: blood lipids, oxidized low-density lipoproteins, lipoprotein(a), homocysteine, and pulmonary nitric oxide: a randomized, controlled, crossover trial. Circulation 106, $1327-1332$ 
100. Gotto JAM (2007) Role of C-reactive protein in coronary risk reduction: focus on primary prevention. Am J Cardiol 99, $718-725$.

101. Maranhão PA, Kraemer-Aguiar LG, de Oliveira CL, et al. (2011) Brazil nuts intake improves lipid profile, oxidative stress and microvascular function in obese adolescents: a randomized controlled trial. Nutr Metab 8, 32

102. Mathieu P, Lemieux I \& Després JP (2010) Obesity, inflammation, and cardiovascular risk. Clin Pharmacol Ther 87, $407-416$

103. United States Food and Drug Administration (2003) Qualified claims about cardiovascular disease risk. Nuts and heart disease. http://www.fda.gov/Food/Ingredients PackagingLabeling/LabelingNutrition/ucm073992.htm\#nuts (accessed October 2013)

104. West SG, Holub BJ, Kris-Etherton PM, et al. (2010) Effects of diets high in walnuts and flax oil on hemodynamic responses to stress and vascular endothelial function. $J \mathrm{Am}$ Coll Nutr 29, 595-603.

105. Adams MR, Kinlay S, Blake GJ, et al. (2000) Atherogenic lipids and endothelial dysfunction: mechanisms in the genesis of ischemic syndromes. Annu Rev Med 51, 149-167.

106. López-Uriarte P, Nogués R, Saez G, et al. (2010) Effect of nut consumption on oxidative stress and the endothelial function in metabolic syndrome. Clin Nutr 29, 373-380.

107. West SG, Gebauer SK, Kay CD, et al. (2012) Diets containing pistachios reduce systolic blood pressure and peripheral vascular responses to stress in adults with dyslipidemia. Hypertension 60, 58-63.

108. Ghiadoni L, Taddei S \& Virdis A (2012) Hypertension and endothelial dysfunction: a therapeutic approach. Curr Vasc Pharmacol 10, 42-60.

109. Din JN, Aftab SM, Jubb AW, et al. (2011) Effect of moderate walnut consumption on lipid profile, arterial stiffness and platelet activation in humans. Eur J Clin Nutr 65, 234-239.

110. Nooyens ACJ, Bueno-de-Mesquita HB, van Boxtel MPJ, et al. (2011) Fruit and vegetable intake and cognitive decline in middle-aged men and women: the Doetinchem Cohort Study. Br J Nutr 106, 752-761.

111. Nurk E, Refsum H, Drevon CA, et al. (2010) Cognitive performance among the elderly in relation to the intake of plant foods. The Hordaland Health Study. Br J Nutr 104, 1190-1201.

112. Pribis P, Bailey RN, Russell AA, et al. (2012) Effects of walnut consumption on cognitive performance in young adults. Br J Nutr 107, 1393-1401.

113. Winnik S, Matter CM, Lohmann C, et al. (2010) Dietary $\alpha$-linolenic acid (ALA) diminishes atherogenesis and restricts $\mathrm{T}$ cell-driven inflammation. Eur Heart $J$ 31, $249-250$.

114. Stark AH, Crawford MA \& Reifen R (2008) Update on $\alpha$-linolenic acid. Nutr Rev 66, 326-332.

115. Freemantle E, Vandal M, Tremblay-Mercier J, et al. (2006) Omega-3 fatty acids, energy substrates, and brain function during aging. Prostaglandins Leukot Essent Fatty Acids 75, $213-220$

116. Risérus U (2008) Fatty acids and insulin sensitivity. Curr Opin Clin Nutr Metab Care 11, 100-105.

117. Salmerón J, Hu FB, Manson JE, et al. (2001) Dietary fat intake and risk of type 2 diabetes in women. Am J Clin Nutr 73, 1019-1026.

118. Vessby B, Uusitupa M, Hermansen K, et al. (2001) Substituting dietary saturated for monounsaturated fat impairs insulin sensitivity in healthy men and women: the KANWU study. Diabetologia 44, 312-319.

119. Dangardt F, Chen Y, Gronowitz E, et al. (2012) High physiological omega-3 fatty acid supplementation affects muscle fatty acid composition and glucose and insulin homeostasis in obese adolescents. J Nutr Metab 2012, 395757.

120. Ebbesson SOE, Tejero ME, López-Alvarenga JC, et al. (2010) Individual saturated fatty acids are associated with different components of insulin resistance and glucose metabolism: the GOCADAN study. Int I Circumpolar Health 69, 344-351.

121. Fuentes F, López-Miranda J, Sánchez E, et al. (2001) Mediterranean and low-fat diets improve endothelial function in hypercholesterolemic men. Ann Intern Med 134, $1115-1119$

122. Solfrizzi V, Colacicco AM, D'Introno A, et al. (2006) Dietary intake of unsaturated fatty acids and age-related cognitive decline: a 8.5-year follow-up of the Italian Longitudinal Study on Aging. Neurobiol Aging 27, 1694-1704.

123. Newens KJ, Thompson AK, Jackson KG, et al. (2011) Acute effects of elevated NEFA on vascular function: a comparison of SFA and MUFA. Br J Nutr 105, 1343-1351.

124. Bellido C, López-Miranda J, Pérez-Martínez P, et al. (2006) The Mediterranean and CHO diets decrease VCAM- 1 and E-selectin expression induced by modified low-density lipoprotein in HUVECs. Nutr Metab Cardiovasc Dis 16, 524-530.

125. Heffernan KS, Patvardhan EA, Ranadive SM, et al. (2010) Review Article: l-arginine as a nutritional prophylaxis against vascular endothelial dysfunction with aging. $J$ Cardiovasc Pharmacol Ther 15, 17-23.

126. Clarkson P, Deanfield JE, Adams MR, et al. (1996) Oral l-arginine improves endothelium-dependent dilation in hypercholesterolemic young adults. J Clin Invest $\mathbf{9 7}$, 1989-1994.

127. Sanders TH, McMichael JRW \& Hendrix KW (2000) Occurrence of resveratrol in edible peanuts. J Agric Food Chem 48, 1243-1246.

128. Ghosh D (2009) Potential role of polyphenol-fortified foods and beverages on vascular health. Agro Food Ind Hi Tech 20, 25-26.

129. Wong RHX, Buckley JD, Coates AM, et al. (2011) Acute resveratrol supplementation improves flow-mediated dilatation in overweight/obese individuals with mildly elevated blood pressure. Nutr Metab Cardiovasc Dis 21, 851-856.

130. Bodinham CL, Smith L \& Wright J (2012) Dietary fibre improves first-phase insulin secretion in overweight individuals. PLOS ONE 7, e40834.

131. Weickert MO, Mohlig M, Schofl C, et al. (2006) Cereal fiber improves whole-body insulin sensitivity in overweight and obese women. Diabetes Care 29, 775-780.

132. Sacks FM, Svetkey LP, Vollmer WM, et al. (2001) Effects on blood presure of reduced dietary sodium and the Dietary Approaches to Stop Hypertension (DASH) diet. $N$ Engl J Med 344, 3-10.

133. Nielsen FH (2010) Magnesium, inflammation, and obesity in chronic disease. Nutr Rev 68, 333-340.

134. Dong J-Y, Xun P, He K, et al. (2011) Magnesium intake and risk of type 2 diabetes: meta-analysis of prospective cohort studies. Diabetes Care 34, 2116-2122.

135. Landmesser U, Hornig B \& Drexler H (2004) Endothelial function: a critical determinant in atherosclerosis? Circula tion 109, Suppl. 1, II27-II33.

136. de Lorgeril M, Boucher F, de Leiris J, et al. (2001) Potential use of nuts for the prevention and treatment of coronary heart disease: from natural to functional foods. Nutr Metab Cardiovasc Dis 11, 362-371.

137. Jackson PA, Reay JL, Scholey AB, et al. (2012) Docosahexaenoic acid-rich fish oil modulates the cerebral hemodynamic response to cognitive tasks in healthy young adults. Biol Psychol 89, 183-190. 
138. Fisher NDL, Sorond FA \& Hollenberg NK (2006) Cocoa flavanols and brain perfusion. I Cardiovasc Pharmacol 47, S210-S214.

139. Wong RHX, Berry NM, Buckley JD, et al. (2012) Regular consumption of a wild green oat extract enhances systemic and cerebral vasodilator function. Hypertension $\mathbf{6 0}$, 498-499.

140. US Department of Agriculture (2011) Agricultural Research Service USDA National Nutrient Database for Standard Reference, Release 26, Nutrient Laboratory Data Home Page. http://www.ars.usda.gov/ba/bhnrc/ndl (accessed October 2013)

141. Kornsteiner M \& Wagner K-H (2006) \& Elmadfa I Tocopherols and total phenolics in 10 different nut types. Food Chem 98, 381-387.

142. Yang J \& Liu RH (2009) \& Halim L Antioxidant and antiproliferative activities of common edible nut seeds. Food Sci Technol 42, 1-8.

143. Tokuşoglu O, Unal MK \& Yemiş F (2005) Determination of the phytoalexin resveratrol (3,5,4'-trihydroxystilbene) in peanuts and pistachios by high-performance liquid chromatographic diode array (HPLC-DAD) and gas chromatography-mass spectrometry (GC-MS). J Agric Food Chem 53, 5003-5009.

144. Blomhoff R, Andersen LF, Carlsen MH, et al. (2006) Health benefits of nuts: potential role of antioxidants. Br J Nutr $\mathbf{9 6}$, S52-S60.

145. Nettleton JA, Steffen LM, Ni H, et al. (2008) Dietary patterns and risk of incident type 2 diabetes in the Multi-Ethnic Study of Atherosclerosis (MESA). Diabetes Care $\mathbf{3 1}$ $1777-1782$

146. Spaccarotella KJ, Kris-Etherton PM, Stone WL, et al. (2008) The effect of walnut intake on factors related to prostate and vascular health in older men. Nutr J 7, 13-23.

147. Iwamoto M, Imaizumi K, Sato M, et al. (2002) Serum lipid profiles in Japanese women and men during consumption of walnuts. Eur J Clin Nutr 56, 629-637.

148. Hiraoka-Yamamoto J, Tashiro M, Miki T, et al. (2004) Serum lipid effects of a monounsaturated (palmitoleic) fatty acid rich diet based on macadamia nuts in healthy, young Japanese women. Clin Exp Pharmacol Physiol 31, S37-S38.

149. Damasceno NRT, Pérez-Heras A, Serra M, et al. (2011) Crossover study of diets enriched with virgin olive oil, walnuts or almonds. Effects on lipids and other cardiovascular risk markers. Nutr Metab Cardiovasc Dis 21, S14-S20.

150. Olmedilla-Alonso B, Granado-Lorencio F, HerreroBarbudo C, et al. (2008) Consumption of restructured meat products with added walnuts has a cholesterollowering effect in subjects at high cardiovascular risk: a randomised, crossover, placebo-controlled study. J Am Coll Nutr 27, 342-348.

151. Nouran MG, Kimiagar M, Abadi A, et al. (2010) Peanut consumption and cardiovascular risk. Public Health Nutr 13, 1581-1586.

152. Sabate J, Fraser GE, Burke K, et al. (1993) Effects of walnuts on serum lipid levels and blood pressure in normal men. $N$ Engl J Med 328, 603-607.

153. Edwards K, Kwaw I, Matud J, et al. (1999) Effect of pistachio nuts on serum lipid levels in patients with moderate hypercholesterolemia. J Am Coll Nutr 18, 229-232.

154. Spiller GA, Miller A, Olivera K, et al. (2003) Effects of plantbased diets high in raw or roasted almonds, or roasted almond butter on serum lipoproteins in humans. $J \mathrm{Am}$ Coll Nutr 22, 195-200.

155. Schutte AE, Van Rooyen JM, Huisman HW, et al. (2006) Modulation of baroreflex sensitivity by walnuts versus cashew nuts in subjects with metabolic syndrome. $A m J$ Hypertens 19, 629-636.

156. Sheridan M, Cooper JN, Erario MJ, et al. (2007) Pistachio nut consumption and serum lipid levels. J Am Coll Nutr 26, 141-148.

157. Kurlandsky SB \& Stote KS (2006) Cardioprotective effects of chocolate and almond consumption in healthy women. Nutr Res 26, 509-516.

158. Valls-Pedret C, Lamuela-Raventos RM, Medina-Remon A, et al. (2012) Polyphenol-rich foods in the Mediterranean diet are associated with better cognitive function in elderly subjects at high cardiovascular risk. J Alzheimers Dis 29, $773-782$ 